\title{
Julia sets in parameter spaces
}

\author{
Buff, X.; Henriksen, C.
}

\section{Published in:}

Communications in Mathematical Physics

Link to article, DOI:

10.1007/PL00005568

Publication date:

2001

\section{Document Version}

Peer reviewed version

Link back to DTU Orbit

\section{Citation (APA):}

Buff, X., \& Henriksen, C. (2001). Julia sets in parameter spaces. Communications in Mathematical Physics, 220(2), 333-375. https://doi.org/10.1007/PL00005568

\section{General rights}

Copyright and moral rights for the publications made accessible in the public portal are retained by the authors and/or other copyright owners and it is a condition of accessing publications that users recognise and abide by the legal requirements associated with these rights.

- Users may download and print one copy of any publication from the public portal for the purpose of private study or research.

- You may not further distribute the material or use it for any profit-making activity or commercial gain

- You may freely distribute the URL identifying the publication in the public portal

If you believe that this document breaches copyright please contact us providing details, and we will remove access to the work immediately and investigate your claim 
Communications in Mathematical Physics manuscript No.

(will be inserted by the editor)

\title{
Julia sets in parameter spaces. *
}

\author{
Xavier Buff ${ }^{1}$, Christian Henriksen ${ }^{2}$ \\ 1 Université Paul Sabatier, UFR MIG, Laboratoire E. Picard, 31062 Toulouse Cedex, France. \\ E-mail: buff@picard.ups-tlse.fr \\ 2 Technical University of Denmark, Department of Mathematics, 2800 Lyngby, Denmark. E- \\ mail: chris@mat.dtu.dk
}

Received: date / Accepted: date

\begin{abstract}
Given a complex number $\lambda$ of modulus 1, we show that the bifurcation locus of the one parameter family $\left\{f_{b}(z)=\lambda z+b z^{2}+z^{3}\right\}_{b \in \mathbb{C}}$ contains quasi-conformal copies of the quadratic Julia set $J\left(\lambda z+z^{2}\right)$. As a corollary, we show that when the Julia set $J\left(\lambda z+z^{2}\right)$ is not locally connected (for example when $z \mapsto \lambda z+z^{2}$ has a Cremer point at 0 ), the bifurcation locus is not locally connected. To our knowledge, this is the first example of complex analytic parameter space of dimension 1, with connected but non-locally connected bifurcation locus. We also show that the set of complex numbers $\lambda$ of modulus 1 , for which at least one of the parameter rays has a non-trivial accumulation set, contains a dense $G_{\delta}$ subset of $S^{1}$.
\end{abstract}

\section{Contents}

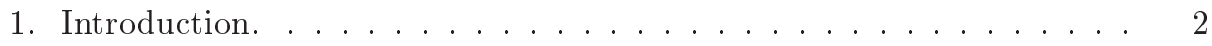

2. Conformal representation of $\overline{\mathbb{C}} \backslash M_{\lambda} \ldots \ldots \ldots \ldots$

3. Copies of quadratic Julia sets in the dynamical plane. . . . . . . . . . 11

4. Definition of the wake $\mathcal{W}_{0} \ldots \ldots \ldots \ldots \ldots \ldots$

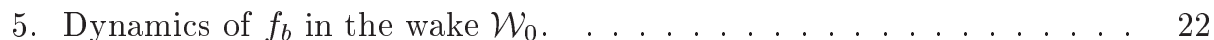

6. Holomorphic motion of rays. . . . . . . . . . . . . . 26

7. The dyadic wakes $\mathcal{W}_{\vartheta} \ldots \ldots \ldots \ldots \ldots \ldots \ldots$

8. Copies of quadratic Julia sets in the parameter plane. . . . . . . . . 37

9. Non local connectivity in the parameter plane. . . . . . . . . . . 40

* Research partially supported by the french embassy in Denmark within the research cooperation between France and Denmark: "Holomorphic Dynamics". 


\section{Introduction.}

In this article, we study the one parameter family of cubic polynomials

$$
f_{b}(z)=\lambda z+b z^{2}+z^{3}, \quad b \in \mathbb{C},
$$

where $\lambda=e^{2 i \pi \theta}$ is a fixed complex number of modulus 1 . We call $K\left(f_{b}\right)$ the filledin Julia set of the polynomial $f_{b}, J\left(f_{b}\right)$ its Julia set, and $M_{\lambda}$ the connectedness locus of the family:

$$
\begin{aligned}
K\left(f_{b}\right) & =\left\{z \in \mathbb{C} \mid\left(f_{b}^{\circ n}(z)\right)_{n \in \mathbb{N}} \text { is bounded }\right\} \\
J\left(f_{b}\right) & =\partial K\left(f_{b}\right), \quad \text { and } \\
M_{\lambda} & =\left\{b \in \mathbb{C} \mid J\left(f_{b}\right) \text { is connected }\right\} .
\end{aligned}
$$

The notations $K_{b}$ and $J_{b}$ are kept for other purposes.

In sections 2 and 3 , we recall some classical results related to the study of the dynamics of cubic polynomials. Those results can be found in [BH1]. In particular, we prove that the connectedness locus $M_{\lambda}$ is connected and we construct dynamically a conformal representation $\Phi_{\lambda}: \mathbb{C} \backslash M_{\lambda} \rightarrow \mathbb{C} \backslash \overline{\mathbb{D}}$ (compare with [Z1]). This enables us to define the parameter rays $\mathcal{R}_{\lambda}(\theta), \theta \in \mathbb{R} / \mathbb{Z}$.

In section 4 , we prove that the parameter rays $\mathcal{R}_{\lambda}(1 / 6)$ and $\mathcal{R}_{\lambda}(1 / 3)$ land at a common parameter $b_{0}$. The techniques we use are not new. They are similar to those developed by Douady and Hubbard in [DH1] to study the landing properties of parameter rays in the quadratic family $\left\{z \mapsto z^{2}+c\right\}_{c \in \mathbb{C}}$. We then define the wake $\mathcal{W}_{0}$ as the connected component of $\mathbb{C} \backslash\left(\overline{\mathcal{R}_{\lambda}(1 / 6)} \cup \overline{\mathcal{R}_{\lambda}(1 / 3)}\right)$ that contains the parameter ray $\mathcal{R}_{\lambda}(1 / 4)$ (see figure 5 ). In section 5 , we study the dynamical features of the polynomials $f_{b}$ when the parameter $b$ ranges in the wake $\mathcal{W}_{0}$.

Matters get interesting in section 6 . Let us define $\Theta \subset \mathbb{R} / \mathbb{Z}$ (respectively $\Theta^{\prime} \subset \mathbb{R} / \mathbb{Z}$ ) to be the Cantor set of angles that can be written in base 3 with only 0 's and 1's (respectively with only 1's and 2's). We denote by $X_{b}$ the set of dynamical rays whose arguments belong to $\Theta$. In section 6 , we prove that the set $X_{b}$ moves holomorphically as long as the parameter $b$ remains in the wake $\mathcal{W}_{0}$. As a consequence, we show that for any parameter $b \in \mathcal{W}_{0}$, the filled-in Julia set $K\left(f_{b}\right)$ contains a quasi-conformal copy of the filled-in Julia set $K\left(\lambda z+z^{2}\right)$ (see figure 11).

Theorem A. For any parameter $b \in \mathcal{W}_{0}$ and for any $\theta \in \Theta$, the dynamical ray $R_{b}(\theta)$ does not bifurcate. We define $X_{b}$ to be the set

$$
X_{b}=\bigcup_{\theta \in \Theta} R_{b}(\theta)
$$

We also define $J_{b}$ to be the set $J_{b}=\overline{X_{b}} \backslash X_{b}$ and $K_{b}$ to be the complement of the unbounded connected component of $\mathbb{C} \backslash J_{b}$. Then, $K_{b}$ is contained in the filled-in Julia set $K\left(f_{b}\right)$, its boundary $J_{b}$ is contained in the Julia set $J\left(f_{b}\right)$ and $K_{b}$ is quasi-conformally homeomorphic to the filled-in Julia set $K\left(\lambda z+z^{2}\right)$.

In the wake $\mathcal{W}_{0}$, one can see a copy $M^{\prime}$ of a Mandelbrot set (see Figure 1). We give a precise definition of the set $M^{\prime}$, but we do not prove that it is 


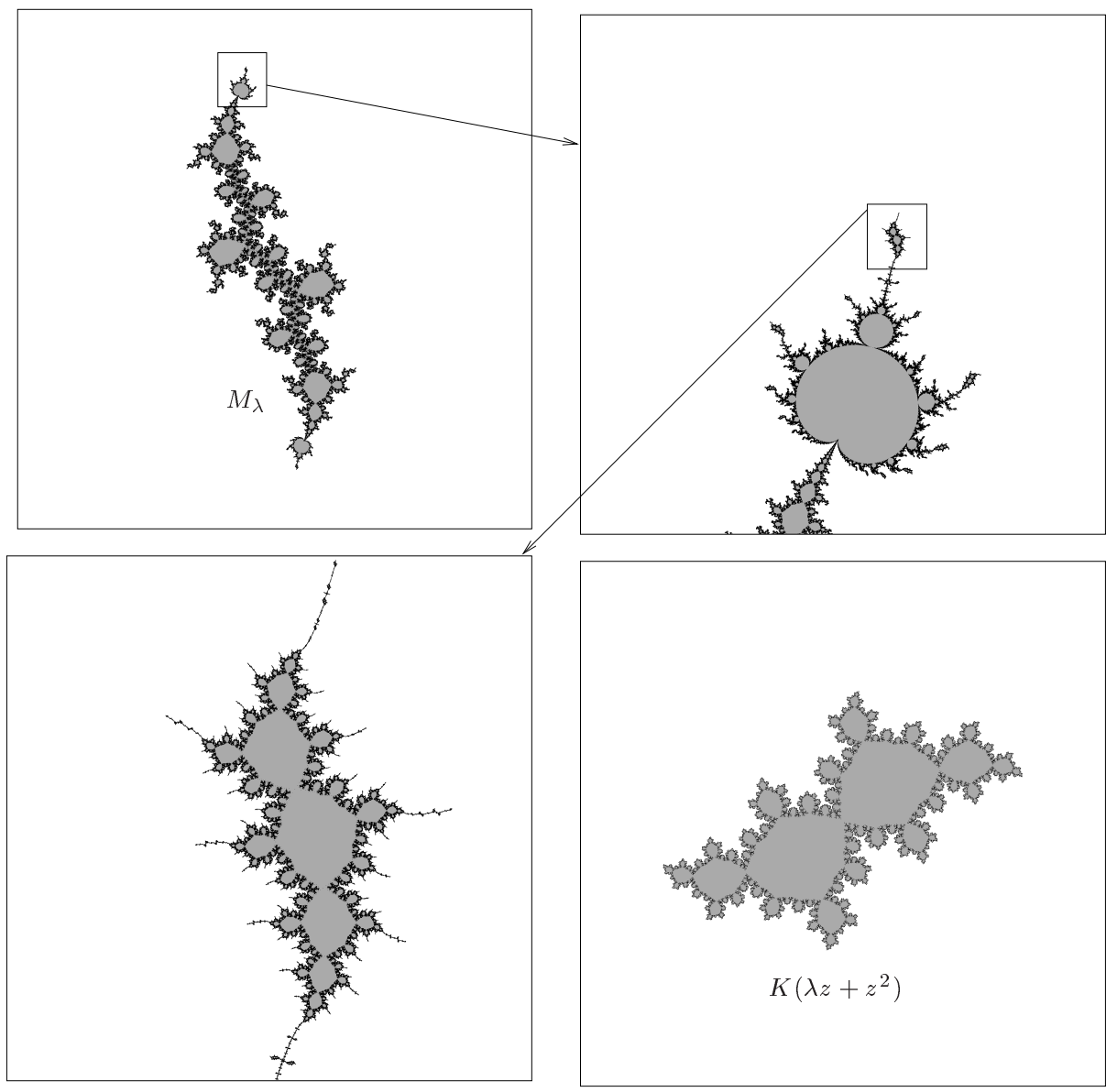

Fig. 1. Zooms in $M_{\lambda}$ for $\lambda=e^{i \pi(\sqrt{5}-1)}$.

homeomorphic to the Mandelbrot set. This has been done in [EY] in the case $\lambda \neq 1$, and is not known in the case $\lambda=1$. However, we show that the boundary of $M^{\prime}$ is equal to the accumulation set of the parameter rays $\mathcal{R}_{\lambda}(\theta / 3), \theta \in \Theta^{\prime}$ (see figure 12):

$$
\partial M^{\prime}=\overline{\mathcal{X}^{\prime}} \backslash \mathcal{X}^{\prime}, \quad \text { where } \quad \mathcal{X}^{\prime}=\bigcup_{\theta \in \Theta^{\prime}} \mathcal{R}_{\lambda}(\theta / 3) .
$$

At the same time, we show that the connected components of $\mathcal{W}_{0} \backslash \overline{\mathcal{X}^{\prime}}$ can be indexed by dyadic angles $\vartheta \in \mathbb{R} / \mathbb{Z}$. The connected component $\mathcal{W}_{\vartheta}$ is bounded by two parameter rays $\mathcal{R}_{\lambda}\left(\vartheta^{-}\right)$and $\mathcal{R}_{\lambda}\left(\vartheta^{+}\right)$landing at a common parameter $b_{\vartheta} \in M^{\prime}$. The angles $\vartheta^{-}$and $\vartheta^{+}$are two consecutive endpoints of the Cantor set $\Theta^{\prime}$. We prove that given any dyadic angle $\vartheta=(2 p+1) / 2^{k}$, we have $\vartheta^{+}=$ $\vartheta^{-}+1 /\left(2 \cdot 3^{k+1}\right)$. We then define the sets $\mathcal{X}_{\vartheta}, \mathcal{J}_{\vartheta}$ and $\mathcal{K}_{\vartheta}$ in the following way:

$$
\mathcal{X}_{\vartheta}=\bigcup_{\theta \in \Theta} \mathcal{R}_{\lambda}\left(\frac{\vartheta^{-}}{3}+\frac{\theta}{3^{k+1}}\right)
$$


$\mathcal{J}_{\vartheta}=\overline{\mathcal{X}_{\vartheta}} \backslash \mathcal{X}_{\vartheta}$, where the closure is taken in $\mathbb{C}$, and $\mathcal{K}_{\vartheta}$ is the complement of the unbounded connected component of $\mathbb{C} \backslash \mathcal{J}_{\vartheta}$. Our main results are the following (see Figure 1).

Main Theorem. Let $\lambda \in S^{1}$ be a complex number of modulus 1 and $\vartheta \in \mathbb{R} / \mathbb{Z}$ be a dyadic angle. The set $\mathcal{K}_{\vartheta}$ is contained in $M_{\lambda} \cap \overline{\mathcal{W}_{\vartheta}}$, its boundary $\mathcal{J}_{\vartheta}$ is contained in the boundary of $M_{\lambda}$ and the parameter $b_{\vartheta}$ belongs to $\mathcal{J}_{\vartheta}$. Besides, there exists a quasi-conformal homeomorphism defined in a neighborhood of $\mathcal{K}_{\vartheta}$, sending $\mathcal{K}_{\vartheta}$ to $K\left(\lambda z+z^{2}\right)$.

Corollary A. For each complex number $\lambda$ of modulus 1 , the bifurcation locus of the one parameter family $f_{b}(z)=\lambda z+b z^{2}+z^{3}, b \in \mathbb{C}$, contains quasi-conformal copies of the quadratic Julia set $J\left(\lambda z+z^{2}\right)$.

Corollary B. If the Julia $J\left(\lambda z+z^{2}\right)$ is not locally connected, then the bifurcation locus $\partial M_{\lambda}$ is not locally connected.

We would like to mention that one has to be careful. Indeed, in the context of Newton's method of cubic polynomials, Pascale Roesch [R] has an example of a locally connected Julia set containing a copy of a quadratic Julia set which is not locally connected. In our case, this does not occur because the set $M_{\lambda}$ is full.

Observe that when $t \in \mathbb{R} \backslash \mathbb{Q}$ does not satisfy the Bruno condition, the quadratic Julia set $J\left(e^{2 i \pi t} z+z^{2}\right)$ is known to be non-locally connected. Hence, the set of values of $\lambda \in S^{1}$ for which $M_{\lambda}$ is not locally connected contains a dense $G_{\delta}$ subset of $S^{1}$. Lavaurs [La] proved that the connectedness locus of the whole family of cubic polynomials is not locally connected. In the parameter space of real cubic polynomials, the bifurcation locus is also known to be non-locally connected (see $[\mathrm{EY}]$ ). To our knowledge, we give the first example of complex parameter space of dimension 1 with connected but non-locally connected bifurcation locus.

Shizuo Nakane brought to our attention that we could prove the existence of parameter rays with a non-trivial accumulation set. He has already proved this result in the family of real cubic polynomials in a joint work with Y. Komori (see $[\mathrm{NK}]$ ). To state the next corollary, we need to introduce some notations.

Given any complex number $\lambda$ of modulus 1 , we define $P_{\lambda}$ to be the quadratic polynomial $P_{\lambda}(z)=\lambda z+z^{2}$. For any angle $\theta \in \mathbb{R} / \mathbb{Z}$, we define $R_{P_{\lambda}}(\theta)$ to be the dynamical ray of the polynomial $P_{\lambda}$ of angle $\theta$. We also consider the Cantor map (or devil staircase) $\chi_{\Theta}: \mathbb{R} / \mathbb{Z} \rightarrow \mathbb{R} / \mathbb{Z}$ which is constant on the closure of each connected component of $\mathbb{R} / \mathbb{Z} \backslash \Theta$ and is defined on $\Theta$ by:

$$
\chi_{\Theta}\left(\sum_{i \geq 1} \frac{\varepsilon_{i}}{3^{i}}\right)=\sum_{i \geq 1} \frac{\varepsilon_{i}}{2^{i}}, \quad \text { where } \quad \varepsilon_{i} \in\{0,1\} .
$$

Corollary C. Given any complex number $\lambda$ of modulus 1 , any dyadic angle $\vartheta=(2 p+1) / 2^{k}$ and any angle $\theta \in \Theta$, the accumulation set of the parameter ray 
$\mathcal{R}_{\lambda}\left(\vartheta^{-} / 3+\theta / 3^{k+1}\right)$ is reduced to a point if and only if the accumulation set of the quadratic ray $R_{P_{\lambda}}\left(\chi_{\Theta}(\theta)\right)$ is reduced to a point.

Using an accumulation theorem due to Douady (see $[\mathrm{S} \varnothing]$ ), we then prove that the set of complex numbers $\lambda$ of modulus 1 , for which at least one of the parameter rays $\mathcal{R}_{\lambda}(\theta) \subset \mathbb{C} \backslash M_{\lambda}$ has a non-trivial accumulation set, contains a dense $G_{\delta}$ subset of $S^{1}$.

We would like to make some comments about the choice of the family $f_{b}$. We wanted to work with a family of cubic polynomials having a persistently indifferent fixed point. We decided to put this fixed point at the origin. This condition is achieved, since the map $f_{b}$ has an indifferent fixed point at 0 with multiplier $\lambda$. The reason why we have chosen this parametrization is that the polynomial $f_{b}$ is monic and thus, has a preferred Böttcher coordinate. This will be useful to define a conformal representation $\Phi_{\lambda}: \mathbb{C} \backslash M_{\lambda} \rightarrow \mathbb{C} \backslash \overline{\mathbb{D}}$ in a dynamical way. This is important since we want to be able to transfer results from the dynamical plane to the parameter plane. However, one should observe that the maps $f_{b}$ and $f_{-b}$ are always conjugate by the affine map $z \mapsto-z$. Indeed,

$$
-f_{b}(-z)=-\left(-\lambda z+b z^{2}-z^{3}\right)=f_{-b}(z) .
$$

This explains why parameter pictures are symmetric with respect to the origin.

The central argument we use is inspired from techniques developed by Tan Lei in [TL]. There, she proves that there are similarities between the Mandelbrot set and certain Julia sets. We would also like to mention that Pia Willumsen proved the existence of copies of the quadratic Julia set $J\left(z^{2}-1\right)$ in the parameter space of a well-chosen family of cubic polynomials.

Hubbard made the suggestion that the two dimensional connectedness locus of the space of cubic polynomials may contain homeomorphic copies of the set

$$
\left\{(c, z) \mid K\left(z^{2}+c\right) \text { is connected and } z \in K\left(z^{2}+c\right)\right\} .
$$

After we exposed our results in Crete ${ }^{1}$, Lyubich and McMullen made the observation that pushing further our arguments, we should be able to prove this result. This would show the existence of cubic polynomials being in the same combinatorial class, but not being topologically conjugate. Such a result has been conjectured by Kiwi in his thesis [K].

\section{Conformal representation of $\overline{\mathbb{C}} \backslash M_{\lambda}$.}

In this section, we will use results by Branner and Hubbard [BH1] to prove that $M_{\lambda}$ is full, connected and has capacity $3 / \sqrt[3]{4}$. We will construct, in a dynamical way, the Riemann mapping $\Phi_{\lambda}: \overline{\mathbb{C}} \backslash M_{\lambda} \rightarrow \overline{\mathbb{C}} \backslash \overline{\mathbb{D}}$, that is tangent to $b \mapsto b \cdot \sqrt[3]{4} / 3$ at infinity. A similar study has already been done by Zakeri [Z1]. Working with the escaping critical value, he defines an analytic map from $\mathbb{C} \backslash M_{\lambda}$ to $\mathbb{C} \backslash \overline{\mathbb{D}}$ which turns out to be a covering map of degree 3 . We will instead work with the escaping co-critical point. We will need this approach later, to transfer dynamical results to the parameter plane. In [Z2], Zakeri also gives an interesting proof of the connectivity of $M_{\lambda}$ based on Teichmüller theory of rational maps.

\footnotetext{
1 Euroconference in Mathematics on Crete; Holomorphic Dynamics; Anogia, June 26 - July 2, 1999 .
} 
2.1. Potential functions. Recall that Fatou proved that the Julia set of any polynomial is connected if and only if the orbit of each critical point is bounded. In our case, the map $f_{b}$ has two critical points. However, $f_{b}$ has an indifferent fixed point at 0 . Hence, there is always one critical point with a bounded orbit. Indeed, there are only three possible cases:

- the fixed point is parabolic $(\theta \in \mathbb{Q})$, and there is at least one critical point of $f_{b}$ in its basin of attraction;

- the fixed point is linearizable (it could be the case even if $\theta$ is not a Bruno number), and the boundary of the Siegel disk is accumulated by the orbit of at least one critical point of $f_{b}$;

- the fixed point is a Cremer point and is contained in the limit set of at least one critical point of $f_{b}$.

Remark. We will say that this critical point is "captured" by 0 .

In particular, when $J\left(f_{b}\right)$ is disconnected, there is exactly one critical point $\omega_{1}$ with bounded orbit, and one escaping critical point $\omega_{2}$.

Let us now recall some classical results that can be found in [DH1] and [BH1].

Definition 1 (Potential functions). For any $b \in \mathbb{C}$, define $g_{b}: \mathbb{C} \rightarrow[0,+\infty[$ by

$$
g_{b}(z)=\lim _{n \rightarrow \infty} \frac{1}{3^{n}} \log ^{+}\left|f_{b}^{\circ n}(z)\right|
$$

where $\log ^{+}$is the supremum of $\log$ and 0 . Also define the function $G: \mathbb{C} \rightarrow \mathbb{R}^{+}$ by

$$
G(b)=\sup _{\left\{\omega \mid f_{b}^{\prime}(\omega)=0\right\}} g_{b}(\omega) .
$$

Remark. When the Julia set $J\left(f_{b}\right)$ is connected, $G(b)=0$. Otherwise, $G(b)=$ $g_{b}\left(\omega_{2}\right)$.

Proposition 1. We have the following properties:

1. $g_{b}$ is continuous and subharmonic on all of $\mathbb{C}$;

2. $g_{b}\left(f_{b}(z)\right)=3 g_{b}(z)$;

3. $g_{b}$ vanishes exactly on $K\left(f_{b}\right)$ and is harmonic on $\mathbb{C} \backslash K\left(f_{b}\right)$;

4. the critical points of $g_{b}$ in $\mathbb{C} \backslash K\left(f_{b}\right)$ are the preimages of the escaping critical point $\omega_{2}$ by an iterate $f_{b}^{\circ n}, n \geq 0$;

5. the mapping $(b, z) \mapsto g_{b}(z)$ is a continuous plurisubharmonic function;

6. the function $G$ is continuous and subharmonic.

Remark. We will see that $G$ vanishes exactly on the set $M_{\lambda}$ and is harmonic outside $M_{\lambda}$.

Definition 2 (Equipotentials). The level curve $g_{b}^{-1}\{\eta\}$ is called the dynamical equipotential of level $\eta$. The level curve $G^{-1}\{\eta\}$ is called the parameter equipotential of level $\eta$.

When the Julia set is connected, the two critical points are contained in $K\left(f_{b}\right)$, and the harmonic map $g_{b}: \mathbb{C} \backslash K\left(f_{b}\right) \rightarrow \mathbb{R}^{+}$has no critical point. Hence, every dynamical equipotential of $f_{b}$ is a real-analytic simple closed curve. More generally, observe that $g_{b}$ has no critical point in the region $\left\{z \in \mathbb{C} \mid g_{b}(z)>\right.$ 
$G(b)\}$, and every dynamical equipotential of level $\eta>G(b)$ is a real-analytic simple closed curve.

The orthogonal curves to dynamical (respectively parameter) equipotentials will be called dynamical (respectively parameter) rays. We will be more precise about the definition of rays below.

Figure 2 shows a filled-in Julia set with two dynamical equipotentials of level $1 / 3$ and 1 , together with four segments of dynamical rays.

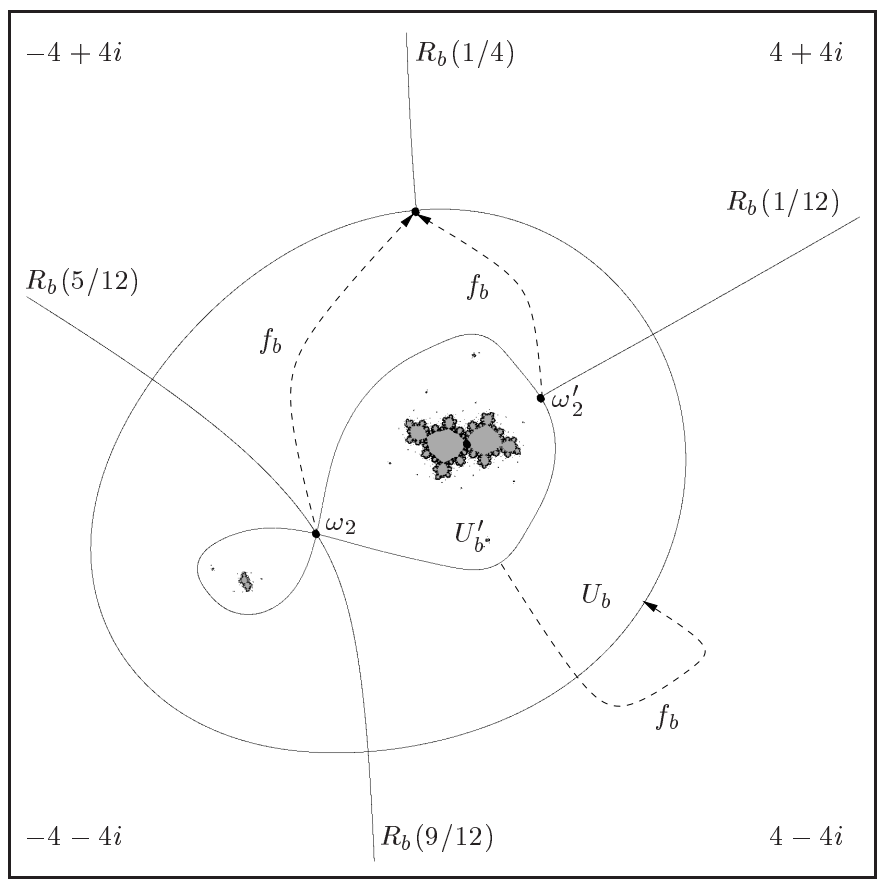

Fig. 2. A disconnected Julia set; $\Phi_{\lambda}(b)=\varphi_{b}\left(\omega_{2}^{\prime}\right)=e^{1 / 3+2 i \pi / 12}$.

2.2. The Böttcher coordinate at infinity.. The vector field

$$
\xi_{b}=\frac{1}{2} \operatorname{grad}\left(g_{b}\right) /\left|\operatorname{grad}\left(g_{b}\right)\right|^{2}
$$

is a meromorphic vector field on $\mathbb{C} \backslash K\left(f_{b}\right)$, having poles exactly at the critical points of $g_{b}$ in $\mathbb{C} \backslash K\left(f_{b}\right)$.

Definition 3. We define $S_{b}$ to be the union of the critical points of $g_{b}$ in $\mathbb{C} \backslash$ $K\left(f_{b}\right)$ and their stable manifolds for the vector field $\xi_{b}$. For any $b \in \mathbb{C}$, we define $V_{b}$ to be the open set $\mathbb{C} \backslash\left(K\left(f_{b}\right) \cup S_{b}\right)$.

We have normalized our cubic polynomials so that they are monic. Hence, there exists a unique Böttcher coordinate $\varphi_{b}$ defined in a neighborhood of infinity, 
and tangent to the identity at infinity. Consider the flow $(z, \tau) \mapsto F_{b}(z, \tau)$ of the vector field $\xi_{b}$, where $\tau \in \mathbb{R}$ is a real time. For any point $z \in V_{b}$, we can extend $\varphi_{b}$ at $z$ using the formula $\varphi_{b}(z)=e^{-\tau} \varphi_{b}\left(F_{b}(z, \tau)\right)$, where $\tau \in[0,+\infty[$ is chosen large enough so that $F_{b}(z, \tau) \in U_{b}$. The following proposition is then easily derived from the analyticity of $\xi_{b}$ and its analytic dependence on $b$.

Proposition 2 (Böttcher coordinate). There exists a unique analytic isomorphism $\varphi_{b}$ defined in a neighborhood of infinity, tangent to the identity at infinity, and satisfying

$$
\varphi_{b} \circ f_{b} \circ \varphi_{b}^{-1}(z)=z^{3} .
$$

The mapping $\varphi_{b}$ extends to an analytic isomorphism $\varphi_{b}: V_{b} \rightarrow \mathbb{C}$ and satisfies $\log \left|\varphi_{b}\right|=g_{b}$ on this set. Furthermore, $\varphi_{b}$ depends analytically on b, i.e., the set

$$
\mathcal{V}=\bigcup_{b \in \mathbb{C}}\{b\} \times V_{b}
$$

is open and the mapping $\Phi: \mathcal{V} \rightarrow \mathbb{C}^{2}$ defined by $\Phi(b, z)=\left(b, \varphi_{b}(z)\right)$ is an analytic isomorphism from $\mathcal{V}$ onto its image.

Remark. An easy computation shows that near infinity, we have $\varphi_{b}(z)=z+$ $b / 3+\mathcal{O}(1 /|z|)$.

When $J\left(f_{b}\right)$ is connected, $V_{b}=\mathbb{C} \backslash K\left(f_{b}\right)$ and the Böttcher coordinate $\varphi_{b}$ is a univalent mapping

$$
\varphi_{b}: \mathbb{C} \backslash K\left(f_{b}\right) \rightarrow \mathbb{C} \backslash \overline{\mathbb{D}}
$$

and on $\mathbb{C} \backslash K\left(f_{b}\right)$, we have $g_{b}=\log \left|\varphi_{b}\right|$. In particular, the dynamical equipotential of level $\eta$ is the set

$$
\varphi_{b}^{-1}\left\{e^{\eta+2 i \pi \theta} \mid \theta \in \mathbb{R} / \mathbb{Z}\right\},
$$

i.e., the preimage by $\varphi_{b}$ of the circle of radius $e^{\eta}$ centered at 0 .

When $J\left(f_{b}\right)$ is disconnected this property still holds for equipotentials of level $\eta>G(b)$, i.e., in the region $\left\{z \in \mathbb{C} \mid g_{b}(z)>G(b)\right\}$.

In both cases, the push-forward $\left(\varphi_{b}\right)_{*}\left(\xi_{b}\right)$ is the radial vector field $w \partial / \partial w$. In particular, $\varphi_{b}$ maps every trajectory of the vector field $\xi_{b}$ to a segment of line with constant argument. Hence, $\varphi_{b}\left(V_{b}\right)$ is a star-shaped domain with respect to infinity, i.e., for every angle $\theta \in \mathbb{R} / \mathbb{Z}$, there exists a radius $r(b, \theta) \geq 1 \mathrm{such}$ that $w \in \varphi_{b}\left(V_{b}\right)$ and $\arg (w)=2 \pi \theta$ if and only if $|w|>r(b, \theta)$. Finally, along a trajectory $z(\tau)$ of the vector field $\xi_{b}$, we have $g_{b}(z(\tau))=g_{b}(z(0))+\tau$.

Definition 4 (Dynamical Rays). For any $b \in \mathbb{C}$, the dynamical ray $R_{b}(\theta)$ is defined as

$$
R_{b}(\theta)=\varphi_{b}^{-1}\left\{r e^{2 i \pi \theta} \mid r>r(b, \theta)\right\} .
$$

Remark. The vector field $\xi_{b}=\frac{1}{2} \operatorname{grad}\left(g_{b}\right) /\left|\operatorname{grad}\left(g_{b}\right)\right|^{2}$ can be extended holomorphically to $\overline{\mathbb{C}} \backslash K\left(f_{b}\right)$. Then, it has a sink at infinity and the dynamical rays are exactly the stable manifolds of infinity for the vector field $\xi_{b}$.

When $r(b, \theta)=1$, the accumulation set of a dynamical ray is contained in the Julia set $J\left(f_{b}\right)$. This is true for any angle $\theta \in \mathbb{R} / \mathbb{Z}$ when $J\left(f_{b}\right)$ is connected. If the limit

$$
z_{0}=\lim _{r \searrow 1} \varphi_{b}^{-1}\left(r e^{2 i \pi \theta}\right)
$$


exists, we will say that the dynamical ray $R_{b}(\theta)$ lands at $z_{0}$. When $J\left(f_{b}\right)$ is disconnected and when $r(b, \theta)>1$, then the limit

$$
z_{0}=\lim _{r \searrow r(b, \theta)} \varphi_{b}^{-1}\left(r e^{2 i \pi \theta}\right)
$$

exists and is a critical point $\omega$ of $g_{b}$. In this case, we will say that the dynamical ray $R_{b}(\theta)$ bifurcates on $\omega$.

If $b^{2}=3 \lambda$, then there is unique critical point. This critical point cannot escape (because 0 "captures" a critical point), and $b \in M_{\lambda}$. On the other hand, if $b^{2} \neq 3 \lambda$ and $b \notin M_{\lambda}$, then $f_{b}\left(\omega_{2}\right)$ has a preimage $\omega_{2}^{\prime} \neq \omega_{2}$. Following Branner and Hubbard, we call it the co-critical point to $\omega_{2}$.

Let us observe that $\varphi_{b}$ is well defined at the co-critical point $\omega_{2}^{\prime}$. Indeed, $\omega_{2}^{\prime}$ cannot be a critical point of $g_{b}$ since it is not an inverse image of $\omega_{2}$. Let us consider the trajectory $z(\tau)$ defined by the initial condition $z(0)=\omega_{2}^{\prime}$. We have $g_{b}(z(\tau))=g_{b}\left(\omega_{2}^{\prime}\right)+\tau$. In particular, since the region $\left\{z \in \mathbb{C} \mid g_{b}(z)>g_{b}\left(\omega_{2}^{\prime}\right)\right\}$ does not contain critical points of $g_{b}$ we see that the trajectory $z(\tau)$ is defined on $\left[0,+\infty\left[\right.\right.$. Hence, $\omega_{2}^{\prime}$ belongs to $V_{b}$, and $\varphi_{b}\left(\omega_{2}^{\prime}\right)$ is well defined.

Definition 5. Given $b \in \mathbb{C} \backslash M_{\lambda}$, the escaping critical point is called $\omega_{2}$ and the co-critical point to $\omega_{2}$ is called $\omega_{2}^{\prime}$. We define the mapping $\Phi_{\lambda}: \mathbb{C} \backslash M_{\lambda} \rightarrow \mathbb{C}$ by

$$
\Phi_{\lambda}(b)=\varphi_{b}\left(\omega_{2}^{\prime}\right) .
$$

Proposition 3 (Branner-Hubbard [BH1] and Zakeri [Z1] [Z2]). The set $M_{\lambda}$ is full and connected. Besides, the map $\Phi_{\lambda}: \mathbb{C} \backslash M_{\lambda} \rightarrow \mathbb{C} \backslash \overline{\mathbb{D}}$ is the conformal isomorphism which is tangent to $b \mapsto b \cdot \sqrt[3]{4} / 3$ at infinity.

Proof. We have seen that if $b^{2}=3 \lambda$, then $b \in M_{\lambda}$. Now, if $b^{2} \neq 3 \lambda$, the two critical points are the two distinct roots of the equation $f_{b}^{\prime}(z)=0$, and by the implicit function theorem, we can follow them locally. Hence, we can follow holomorphically the two critical points locally outside $M_{\lambda}$. For the same reason, we can follow holomorphically the two distinct co-critical points locally outside $M_{\lambda}$.

Lemma 1. The mapping $\Phi_{\lambda}: \mathbb{C} \backslash M_{\lambda} \rightarrow \mathbb{C} \backslash \overline{\mathbb{D}}$ is analytic.

Proof. Fix a parameter $b_{0} \notin M_{\lambda}$, let $\omega_{2}$ be the escaping critical point and $\omega_{2}^{\prime}$ be the co-critical point to $\omega_{2}$. There exist two holomorphic maps defined in a neighborhood $\mathcal{U}$ of $b_{0}$ that follow the two co-critical points. Let $\omega^{\prime}: \mathcal{U} \rightarrow \mathbb{C}$ be the one which coincide with $\omega_{2}^{\prime}$ at $b_{0}$. The set

$$
\mathcal{W}=\left\{(b, z) \in \mathbb{C}^{2} \mid z \in \mathbb{C} \backslash K\left(f_{b}\right)\right\}
$$

is the preimage of $] 0,+\infty$ [ by the map $g(b, z)=g_{b}(z)$ which is continuous by 5$)$ of proposition 1 . Hence, $\mathcal{W}$ is open. Thus, by restricting $\mathcal{U}$ if necessary, we may assume that for any $b \in \mathcal{U}$, the co-critical point $\omega^{\prime}(b)$ belongs to $\mathbb{C} \backslash K\left(f_{b}\right)$. This shows that, for any $b \in \mathcal{U}$, the escaping co-critical point is $\omega^{\prime}(b)$.

Furthermore, the mapping $(b, z) \mapsto \varphi_{b}(z)$ is analytic in a neighborhood of any point $\left(b_{0}, z_{0}\right)$ such that $z_{0} \in V_{b_{0}}$. Hence, it is analytic in a neighborhood of $\left(b_{0}, \omega_{2}^{\prime}\right)$. It follows immediately that $\Phi_{\lambda}(b)=\varphi_{b}\left(\omega_{2}^{\prime}(b)\right)$ is analytic in a neighborhood of $b_{0}$. 
The proof that $\Phi_{\lambda}$ is an isomorphism between $\mathbb{C} \backslash M_{\lambda}$ and $\mathbb{C} \backslash \overline{\mathbb{D}}$ is an application of the principle: an analytic mapping is an isomorphism if it is proper of degree 1. We shall use a similar argument for quasi-regular mappings in section 8 .

Lemma 2. Outside $M_{\lambda}$, we have $G(b)=\log \left|\Phi_{\lambda}(b)\right|$. Besides, the function $G$ vanishes exactly on $M_{\lambda}$.

Proof. If $b \notin M_{\lambda}$, we can write:

$$
\begin{aligned}
\log \left|\Phi_{\lambda}(b)\right| & =\frac{1}{3^{n}} \log \left|\varphi_{b}\left(f^{\circ n}\left(\omega_{2}^{\prime}\right)\right)\right| \\
& =\frac{1}{3^{n}} \log \left|f^{\circ n}\left(\omega_{2}^{\prime}\right)+\mathcal{O}\left(\frac{1}{\left|f^{\circ n}\left(\omega_{2}^{\prime}\right)\right|}\right)\right|=G(b) .
\end{aligned}
$$

Since $\varphi_{b}$ takes values outside $\overline{\mathbb{D}}$, so does $\Phi_{\lambda}$. Hence, $G$ is positive outside $M_{\lambda}$. Besides, if $b \in M_{\lambda}$, both critical points are in the filled-in Julia set $K\left(f_{b}\right)$. So, their orbits are bounded and $G(b)=0$.

We can now see that $M_{\lambda}$ is full. This is an immediate consequence of the fact that sub-harmonic functions satisfy the maximum principle. Thus, level sets are full.

By Picard's theorem, the mapping $\Phi_{\lambda}: \mathbb{C} \backslash M_{\lambda} \rightarrow \mathbb{C} \backslash \overline{\mathbb{D}}$ has a removable singularity at infinity. Hence, we can extend it to infinity. We necessarily have $\Phi_{\lambda}(\infty)=\infty$ since otherwise $G$ would be a non-constant bounded subharmonic function on $\mathbb{P}^{1}$.

More precisely, a simple computation shows that when $|b|$ tends to infinity,

$$
\omega_{2}=-\frac{2 b}{3}+o(1), \quad \text { and } \quad \omega_{2}^{\prime}=-b-2 \omega_{2}=\frac{b}{3}+o(1) .
$$

Then,

$$
\frac{f_{b}\left(\omega_{2}^{\prime}\right)}{\left(\omega_{2}^{\prime}\right)^{3}}=4+\mathcal{O}\left(\frac{1}{|b|}\right),
$$

and for any integer $n \geq 1$, we have

$$
\frac{f_{b}^{\circ(n+1)}\left(\omega_{2}^{\prime}\right)}{\left(f_{b}^{\circ n}\left(\omega_{2}^{\prime}\right)\right)^{3}}=1+\mathcal{O}\left(\frac{1}{|b|}\right)
$$

Hence, we obtain

$$
\Phi_{\lambda}(b)=\omega_{2}^{\prime} \prod\left(\frac{f_{b}^{\circ(n+1)}\left(\omega_{2}^{\prime}\right)}{\left(f_{b}^{\circ n}\left(\omega_{2}^{\prime}\right)\right)^{3}}\right)^{1 / 3^{n+1}}=\frac{\sqrt[3]{4}}{3} b+\mathcal{O}\left(\frac{1}{|b|}\right) .
$$

We will now show that $\Phi_{\lambda}: \overline{\mathbb{C}} \backslash M_{\lambda} \rightarrow \overline{\mathbb{C}} \backslash \overline{\mathbb{D}}$ is a proper mapping. Since $G$ is continuous, $G(b)$ tends to 0 as $b$ tends to the boundary of $M_{\lambda}$. Hence, $\Phi_{\lambda}(b)$ tends to $\partial \mathbb{D}$ when $b$ tends to $\partial M_{\lambda}$ from outside $M_{\lambda}$. Since $\Phi_{\lambda}$ is analytic, it is a proper mapping.

We can finally see that it has degree 1 since infinity has only one preimage counted with multiplicity. Hence, it is an isomorphism between $\overline{\mathbb{C}} \backslash M_{\lambda}$ and $\overline{\mathbb{C}} \backslash \overline{\mathbb{D}}$. In particular, $M_{\lambda}$ is connected. 
We have defined parameter equipotentials. We can now define parameter rays (see Figure 5).

Definition 6 (Parameter Rays). The parameter ray $\mathcal{R}_{\lambda}(\theta)$ is defined as

$$
\mathcal{R}_{\lambda}(\theta)=\Phi_{\lambda}^{-1}\left\{e^{\eta+2 i \pi \theta} \mid \eta>0\right\} .
$$

If the limit

$$
b_{0}=\lim _{r \searrow 1} \Phi_{\lambda}^{-1}\left(r e^{2 i \pi \theta}\right)
$$

exists, we will say that the parameter ray $\mathcal{R}_{\lambda}(\theta)$ lands at $b_{0}$.

\section{Copies of quadratic Julia sets in the dynamical plane.}

In this section, we will first recall a result which is essentially due to Branner and Hubbard [BH2] (see also [Br]): when the parameter $b$ is not in $M_{\lambda}$, there exists a restriction of $f_{b}$ which is a quadratic-like mapping. The reader will find information on polynomial-like mappings and related results in [DH2].

Definition 7 (Polynomial-like mappings). A polynomial-like mapping $f$ : $U^{\prime} \rightarrow U$ of degree $d$ is a ramified covering of degree d between two topological disks $U^{\prime}$ and $U$, with $U^{\prime}$ relatively compact in $U$. One can define its filled-in Julia set $K(f)$ and its Julia set $J(f)$ as follows:

$$
K(f)=\left\{z \in U^{\prime} \mid(\forall n \in \mathbb{N}) f^{\circ n}(z) \in U^{\prime}\right\}, \quad \text { and } \quad J(f)=\partial K(f) .
$$

A polynomial-like mapping of degree 2 will be called a quadratic-like mapping.

Let us recall the so-called Straightening Theorem due to Douady and Hubbard.

Proposition 4 (Straightening Theorem). If $f: U^{\prime} \rightarrow U$ is a polynomial-like mapping of degree $d$. Then there exists

- a polynomial $P: \mathbb{C} \rightarrow \mathbb{C}$ of degree $d$,

- a neighborhood $V$ of the filled-in Julia set $K(P)$ such that the mapping $P$ :

$P^{-1}(V)=V^{\prime} \rightarrow V$ is a polynomial-like map, and

- a quasiconformal homeomorphism $\varphi: U \rightarrow V$ with $\varphi\left(U^{\prime}\right)=V^{\prime}$, such that $\bar{\partial} \varphi=0$ almost everywhere on $K(f)$ and such that on $U^{\prime}$

$$
\varphi \circ P=f \circ \varphi .
$$

Moreover, if $K(f)$ is connected, then $P$ is unique up to conformal conjugacy.

Definition 8. Two polynomial-like mappings $f$ and $g$ are said to be hybrid equivalent if there is a quasi-conformal $h$ that conjugates $f$ and $g$, with $\bar{\partial} h=0$ almost everywhere on the filled-in Julia set $K(f)$.

Proposition 5. For any $b \in \mathbb{C} \backslash M_{\lambda}$, let us denote by $U_{b}$ the open set $\{z \in$ $\left.\mathbb{C} \mid g_{b}(z)<3 G(b)\right\}$ and $U_{b}^{\prime}$ the connected component of $f^{-1}\left(U_{b}\right)$ that contains the non-escaping critical point $\omega_{1}$. Then, the restriction $f_{b}: U_{b}^{\prime} \rightarrow U_{b}$ is a quadratic-like mapping and its hybrid class contains the polynomial $z \mapsto \lambda z+z^{2}$. 
Figure 2 shows the domains $U_{b}^{\prime}$ and $U_{b}$ for the parameter $\Phi_{\lambda}^{-1}\left(e^{1 / 3+2 i \pi / 12}\right)$. Proof. We have seen that any dynamical equipotential of level $\eta>G(b)$ is a real-analytic simple closed curve. This applies to the dynamical equipotential of level $3 G(b)$. Thus, the set $U_{b}$ is a topological disk. Besides, it only contains one critical value of $f_{b}$ (the non-escaping one). The set $f^{-1}\left(U_{b}\right)$ is the set $\left\{z \in \mathbb{C} \mid g_{b}(z)<G(b)\right\}$ which is bounded by a lemniscate pinching at the escaping critical point $\omega_{2}$. Each connected component of $f^{-1}\left(U_{b}\right)$ is a topological disk compactly contained in $U_{b}$. Besides, the restriction of $f_{b}$ to the connected component of $f^{-1}\left(U_{b}\right)$ containing the non-escaping critical point $\omega_{1}$ is a ramified covering of degree 2, ramified at $\omega_{1}$. This is precisely the definition of a quadratic-like mapping.

Next, to see that the hybrid class of this quadratic-like mapping contains $z \mapsto \lambda z+z^{2}$, we will use the following result.

Lemma 3. The multiplier of an indifferent fixed point is a quasi-conformal invariant.

Remark. Naishül [Nai] shows a much better result since he proves that the multiplier of an indifferent fixed point is a topological invariant. Pérez-Marco [PM] gave a new proof of this result which is much simpler. The case of quasi-conformal conjugacy is easier to handle. R. Douady gave an easy proof based on the compacity of the space of quasi-conformal mappings with bounded dilatation (see $[\mathrm{Y}]$ ). We will present a new proof based on holomorphic motions and the AhlforsBers theorem. Those tools are more complicated than the ones used by Douady, but the idea of the proof fits very well within this article.

Proof. Assume that two germs $f_{0}: U_{0} \rightarrow \mathbb{C}$ and $f_{1}: U_{1} \rightarrow \mathbb{C}$ are quasiconformally conjugate. Call $\psi$ the quasi-conformal conjugacy. Then $\mu=\bar{\partial} \psi / \partial \psi$ is a Beltrami form invariant by $f_{0}$. Integrating the Beltrami form $\mu_{\varepsilon}=\varepsilon \mu$, $\varepsilon \in \mathbb{D}\left(0,1 /\|\mu\|_{\infty}\right)$, we get a family of quasi-conformal homeomorphisms $\psi_{\varepsilon}$ depending analytically on $\varepsilon$, and a family of analytic germs

$$
f_{\varepsilon}=\psi_{\varepsilon} \circ f_{0} \circ \psi_{\varepsilon}^{-1}
$$

We claim that this family of germs depend analytically on $\varepsilon$ (this is not immediate since $\psi_{\varepsilon}^{-1}$ does not need to depend analytically on $\varepsilon$; Douady explained us a geometric proof, and Lyubich explained us an analytic proof which we give here). Since $f_{\varepsilon} \circ \psi_{\varepsilon}=\psi_{\varepsilon} \circ f_{0}$, for any $z \in U$ we can write

$$
\left.\frac{\partial f_{\varepsilon}}{\partial \bar{\varepsilon}}\right|_{\psi_{\varepsilon}(z)}+\left.\frac{\partial f_{\varepsilon}}{\partial \bar{z}} \cdot \frac{\partial \overline{\psi_{\varepsilon}}}{\partial \bar{\varepsilon}}\right|_{z}+\left.\frac{\partial f_{\varepsilon}}{\partial z} \cdot \frac{\partial \psi_{\varepsilon}}{\partial \bar{\varepsilon}}\right|_{z}=\left.\frac{\partial \psi_{\varepsilon}}{\partial \bar{\varepsilon}}\right|_{f_{0}(z)} .
$$

Since both $\partial f_{\varepsilon} / \partial \bar{z}$ and $\partial \psi_{\varepsilon} / \partial \bar{\varepsilon}$ vanish, we see that $\partial f_{\varepsilon} / \partial \bar{\varepsilon}$ vanishes.

In particular, the multiplier $\lambda(\varepsilon)$ of the fixed point depends analytically on $\varepsilon$. Since it cannot become repelling or attracting (all the germs are conjugate to $f_{0}$ which has an indifferent fixed point), the modulus of $\lambda(\varepsilon)$ is constant. Hence, $\lambda(\varepsilon)$ is a constant function, and $\lambda(1)=\lambda(0)$.

The hybrid class of the quadratic-like map $f_{b}: U_{b}^{\prime} \rightarrow U_{b}$ contains a quadratic polynomial having an indifferent fixed point with multiplier $\lambda$. Such a polynomial is always analytically conjugate to the polynomial $z \mapsto \lambda z+z^{2}$. 
Definition 9. For any parameter $b \in \mathbb{C} \backslash M_{\lambda}$, the filled-in Julia set of the quadratic-like map $f_{b}: U_{b}^{\prime} \rightarrow U_{b}$ is called $K_{b}$ and its Julia set is called $J_{b}$.

We will now give more informations about the dynamics of $f_{b_{1}}$ for the parameter $b_{1}$ with potential $\eta=1 / 3$ and external argument $\theta=1 / 4$ (we could have picked any parameter with potential $\eta>0$ and external argument $\theta \in] 1 / 6,1 / 3[$ ).

Proposition 6. Let $b_{1}$ be the parameter $b_{1}=\Phi_{\lambda}^{-1}\left(e^{1 / 3+2 i \pi / 4}\right)$. If $\lambda \neq 1$, the two dynamical rays $R_{b_{1}}(0 / 1)$ and $R_{b_{1}}(1 / 2)$ both land at a common fixed point $\beta \neq 0$ which is repelling. If $\lambda=1$, the rays $R_{b_{1}}(0 / 1)$ and $R_{b_{1}}(1 / 2)$ both land at the parabolic fixed point $\beta=0$.

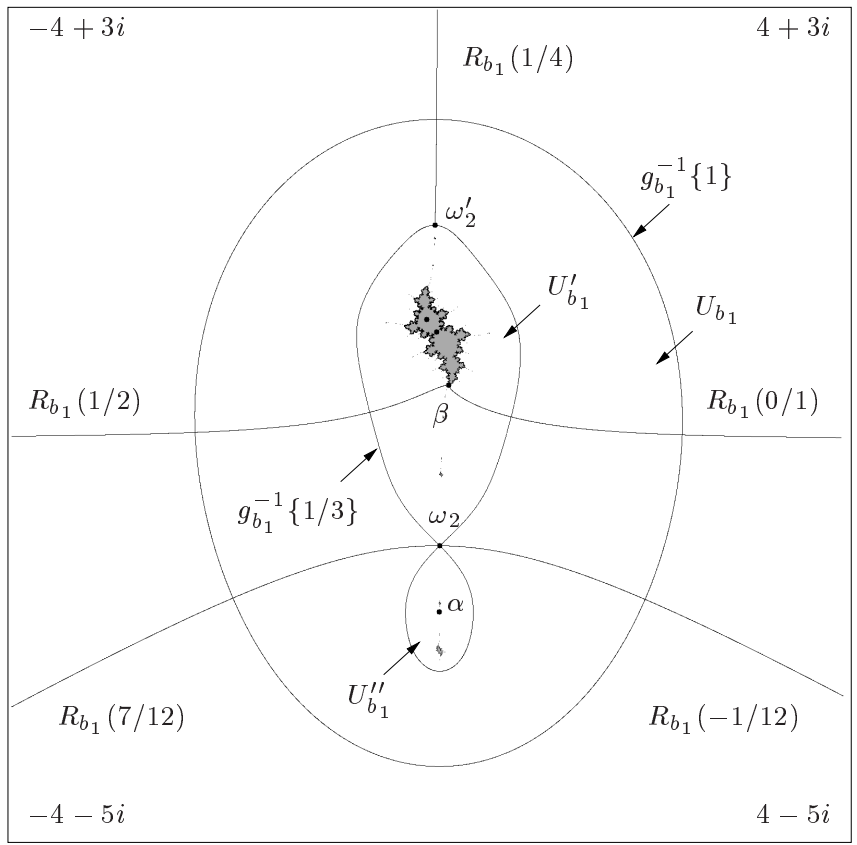

Fig. 3. The rays $R_{b_{1}}(0 / 1)$ and $R_{b_{1}}(1 / 2)$ both land at a common fixed point $\beta$.

Proof. We still denote by $U_{b_{1}}$ the set $U_{b_{1}}=\left\{z \in \mathbb{C} \mid g_{b_{1}}(z)<3 G\left(b_{1}\right)\right\}$. Its preimage $f_{b_{1}}^{-1}\left(U_{b_{1}}\right)$ has two connected components. Note that $U_{b_{1}}^{\prime}$ is the one containing $\omega_{2}^{\prime}$ in its boundary. Denote by $U_{b_{1}}^{\prime \prime}$ the other component (see figure 3). Remember that, $f_{b_{1}}: U_{b_{1}}^{\prime} \rightarrow U_{b_{1}}$ is a degree 2 proper mapping. Similarly $f_{b_{1}}: U_{b_{1}}^{\prime \prime} \rightarrow U_{b_{1}}$ is a degree 1 proper mapping and since $U_{b_{1}}^{\prime \prime}$ is compactly contained in $U_{b_{1}}, f_{b_{1}}$ has exactly one fixed point in $U_{b_{1}}^{\prime \prime}$. This fixed point is repelling. We will denote it by $\alpha$.

Next, observe that the rays $R_{b_{1}}(-1 / 12)$ and $R_{b_{1}}(7 / 12)$ bifurcate on $\omega_{2}$, and since $-1 / 12<0<1 / 2<7 / 12$, they separate $\alpha$ from the rays $R_{b_{1}}(0 / 1)$ and $R_{b_{1}}(1 / 2)$. 
Since $f_{b_{1}}: U_{b_{1}}^{\prime} \rightarrow U_{b_{1}}$ is a degree 2 proper mapping, and since $U_{b_{1}}^{\prime}$ is compactly contained in $U_{b_{1}}$, Rouché's Theorem shows that $f_{b_{1}}$ has exactly two fixed points in $U_{b_{1}}^{\prime}$, counted with multiplicity. If $\lambda \neq 1$, those two fixed points are distinct. One is 0 which is indifferent, and has multiplier $\lambda$, the other one will be denoted by $\beta$. A theorem due to Douady-Hubbard [DH1] and to Sullivan asserts that every fixed dynamical ray that does not bifurcate, lands at a fixed point which is either repelling, or parabolic with multiplier 1 . Since the two fixed dynamical rays $R_{b_{1}}(0 / 1)$ and $R_{b_{1}}(1 / 2)$ cannot land at 0 (since the multiplier is neither repelling nor equal to 1 ), they must both land at the fixed point $\beta$. Since $\beta$ is the landing point of a ray, either it is repelling or it is a multiple fixed point. But since there are only two fixed point in $U_{b_{1}}^{\prime}$ counted with multiplicity the former case occurs.

On the other hand, if $\lambda=1$, there is only one fixed point in $U_{b_{1}}^{\prime}$ : the fixed point at 0 which is parabolic with multiplier 1 . Hence the two fixed rays $R_{b_{1}}(0 / 1)$ and $R_{b_{1}}(1 / 2)$ must both land at 0 .

We will now describe the set of rays that accumulate on the Julia set $J_{b_{1}}$ of the quadratic-like map $f_{b_{1}}: U_{b_{1}}^{\prime} \rightarrow U_{b_{1}}$.

Definition 10. We define $\Theta \subset \mathbb{R} / \mathbb{Z}$ to be the set of angles $\theta$ such that for any $n \geq 0,3^{n} \theta \in[0,1 / 2] \bmod 1$.

Remark. The set $\Theta$ is the set of angles $\theta$ that can be written in base 3 with only 0 's and 1's. It is a Cantor set and is forward invariant under multiplication by 3 .

Figure 4 shows the dynamical rays $R_{b_{1}}(\theta)$ for $\theta \in \Theta$. The following proposition shows that those rays accumulate on the Julia set $J_{b_{1}}$ of the quadratic-like restriction of $f_{b_{1}}$.
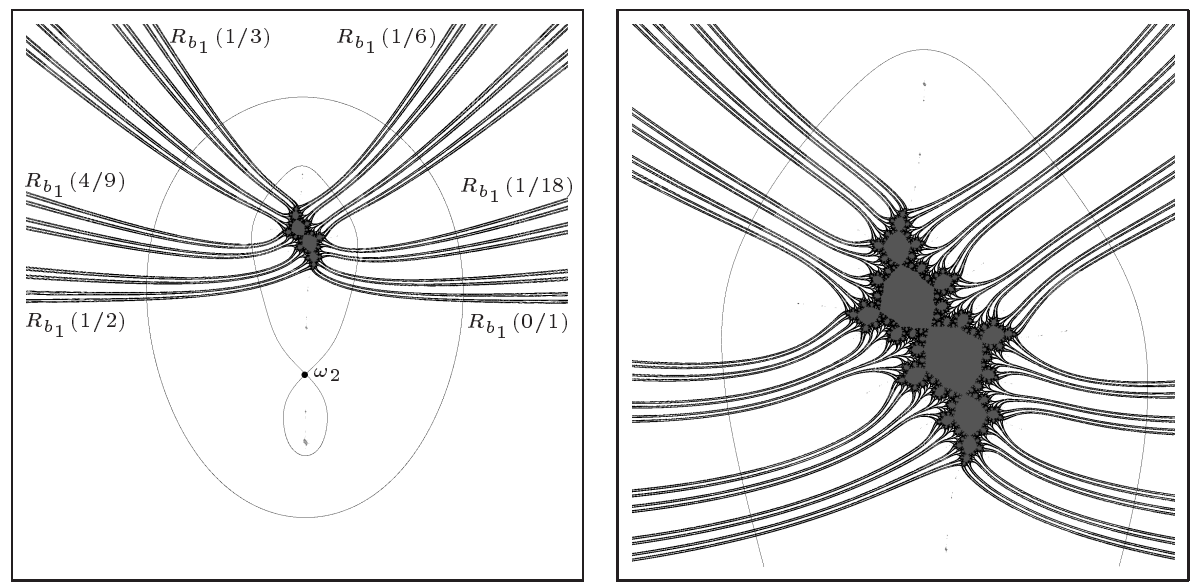

Fig. 4. The dynamical rays $R_{b_{1}}(\theta), \theta \in \Theta$, accumulate on the Julia set $J_{b_{1}}$ of the quadraticlike restriction of $f_{b_{1}}$. 
Proposition 7. Let $b_{1}$ be the parameter $b_{1}=\Phi_{\lambda}^{-1}\left(e^{1 / 3+2 i \pi / 4}\right)$ and $J_{b_{1}}$ be the Julia set of the quadratic-like mapping $f_{b_{1}}: U_{b_{1}}^{\prime} \rightarrow U_{b_{1}}$. Then, for any $\theta \in \Theta$, the dynamical ray $R_{b_{1}}(\theta)$ does not bifurcate. Besides, if we define

$$
X_{b_{1}}=\bigcup_{\theta \in \Theta} R_{b_{1}}(\theta)
$$

then $\overline{X_{b_{1}}} \backslash X_{b_{1}}=J_{b_{1}}$.

Proof. Let us first recall that the rays $R_{b_{1}}(0 / 1)$ and $R_{b_{1}}(1 / 2)$ do not bifurcate and land at the same fixed point $\beta$. Hence, the curve $\{\beta\} \cup R_{b_{1}}(0 / 1) \cup R_{b_{1}}(1 / 2)$ cuts the plane in two connected components $V_{1}$ and $V_{2}$. We call $V_{2}$ the one containing the escaping critical point $\omega_{2}$. Observe that for any $\theta \in[0,1 / 2]$, the dynamical ray $R_{b_{1}}(\theta)$ is contained in $\mathbb{C} \backslash V_{2}$. Now, assume that there exists an angle $\theta \in \Theta$ such that the dynamical ray $R_{b_{1}}(\theta)$ bifurcates. Then, it bifurcates on a preimage of the escaping critical point $\omega_{2}$ and one of its forward image bifurcates on $\omega_{2}$. But since by definition of $\Theta$, we have $3^{k} \theta \in[0,1 / 2] \bmod 1$, for any $k \geq 0$, the forward orbit of the ray $R_{b_{1}}(\theta)$ is contained in $\mathbb{C} \backslash V_{2}$. Hence no forward image of $R_{b_{1}}(\theta)$ can bifurcate on the escaping critical point $\omega_{2} \in V_{2}$.

Since the set $\Theta$ is closed (it is an intersection of closed sets), $X_{b_{1}}$ is closed in $\mathbb{C} \backslash K\left(f_{b_{1}}\right)$. Hence,

$$
\overline{X_{b_{1}}} \backslash X_{b_{1}} \subset J\left(f_{b_{1}}\right) .
$$

We will now show that for any angle $\theta \in \Theta$, the accumulation set $\mathcal{I}$ of the ray $R_{b_{1}}(\theta)$ is contained in the Julia set $J_{b_{1}}$ of the quadratic-like mapping $f_{b_{1}}: U_{b_{1}}^{\prime} \rightarrow$ $U_{b_{1}}$. Indeed, the accumulation set $\mathcal{I}$ is contained in the Julia set $J\left(f_{b_{1}}\right)$ of $f_{b_{1}}$, and its forward orbit is contained in $\mathbb{C} \backslash V_{2}$. In particular, it cannot enter the region $U_{b_{1}}^{\prime \prime}$, and the forward orbit of $\mathcal{I}$ is entirely contained in $U_{b_{1}}^{\prime}$. This shows that $\mathcal{I} \subset K_{b_{1}}$. Since $\mathcal{I}$ is contained in the boundary of $K\left(f_{b_{1}}\right)$, we see that $\mathcal{I} \subset J_{b_{1}}$, and

$$
\overline{X_{b_{1}}} \backslash X_{b_{1}} \subset J_{b_{1}} .
$$

To prove the reverse inclusion, we will use the fact that the backward orbit of the fixed point $\beta$ by the quadratic-like map $f_{b_{1}}: U_{b_{1}}^{\prime} \rightarrow U_{b_{1}}$ is dense in $J_{b_{1}}$. Let us show by induction on $n$ that if $z \in J_{b_{1}}$ satisfies $f_{b_{1}}^{\circ n}(z)=\beta$, then there is an angle $\theta \in \Theta$ such that $R_{b_{1}}(\theta)$ lands at $z$. This is true for $n=0$ since the rays $R_{b_{1}}(0 / 1)$ and $R_{b_{1}}(1 / 2)$ land at $\beta$. Now, if the induction property holds for some $n$, let us show that it is true for $n+1$. Given a point $z \in J_{b_{1}}$ satisfying $f_{b_{1}}^{o(n+1)}(z)=\beta$, its image $f_{b_{1}}(z)$ satisfies the induction hypothesis. Thus, there is an angle $\theta \in \Theta$ such that the ray $R_{b_{1}}(\theta)$ lands at $f_{b_{1}}(z)$. Observe that, on one hand, this ray cannot contain the escaping critical value (indeed, the ray containing the escaping critical value has argument $3 / 4 \notin \Theta)$, and its three preimages land at the three preimages of $f_{b_{1}}(z)$. On the other hand, there are three angles $\theta_{1}, \theta_{2}$ and $\theta_{3}$ such that $3 \theta_{i}=\theta, i=1,2,3$. Two of them, let's say $\theta_{1}$ and $\theta_{2}$, are in $\Theta$, and the third one, $\theta_{3}$, is contained in $] 2 / 3,5 / 6[\bmod 1$. Hence, the ray $R_{b_{1}}\left(\theta_{3}\right)$ lands at the preimage of $f_{b_{1}}(z)$ which is contained in $U_{b_{1}}^{\prime \prime}$. This shows that one of the two rays $R_{b_{1}}\left(\theta_{1}\right)$ or $R_{b_{1}}\left(\theta_{2}\right)$ lands at $z$.

Remark. It is easy to see that no other dynamical ray can accumulate on $J_{b_{1}}$ since their forward orbits eventually enter $V_{2}$. 


\section{Definition of the wake $\mathcal{W}_{0}$.}

We will now restrict our study to a particular region in the parameter plane: the wake $\mathcal{W}_{0}$.

Definition 11. The wake $\mathcal{W}_{0}$ is defined to be the connected component of

$$
\mathbb{C} \backslash \overline{\mathcal{R}_{\lambda}(1 / 6)} \cup \overline{\mathcal{R}_{\lambda}(1 / 3)} \cup \overline{\mathcal{R}_{\lambda}(2 / 3)} \cup \overline{\mathcal{R}_{\lambda}(5 / 6)}
$$

that contains the parameter ray $\mathcal{R}_{\lambda}(1 / 4)$.

Remark. In fact, we will show that the parameter rays $\mathcal{R}_{\lambda}(1 / 6)$ and $\mathcal{R}_{\lambda}(1 / 3)$ land at a common parameter $b_{0}$ which satisfies the equation $b_{0}^{2}=4(\lambda-1)$. The wake $\mathcal{W}_{0}$ is the region contained between those two rays (see Figure 5 ).

There are several ways of proving the landing property of the parameter rays $\mathcal{R}_{\lambda}(1 / 6)$ and $\mathcal{R}_{\lambda}(1 / 3)$. We will use an argument similar to the one used by Douady and Hubbard in [DH1]. We will need to modify it slightly in the case $\lambda=1$.

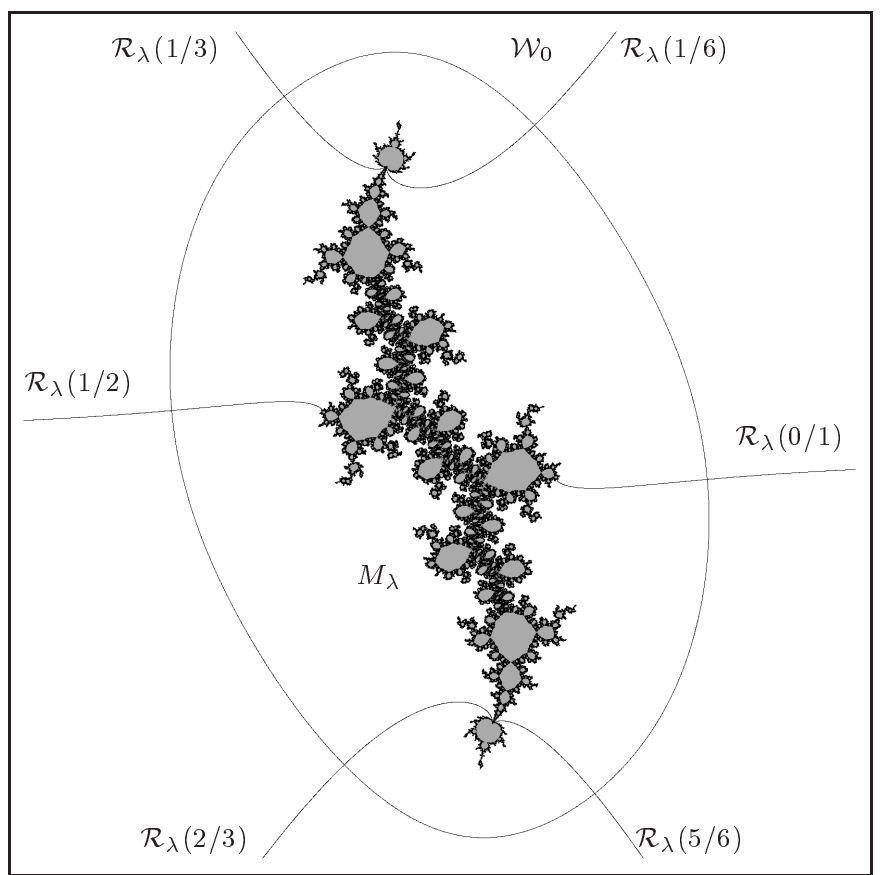

Fig. 5. The parameter rays $\mathcal{R}_{\lambda}(1 / 6)$ and $\mathcal{R}_{\lambda}(1 / 3)$ land at $b_{0}$, whereas the rays $\mathcal{R}_{\lambda}(2 / 3)$ and $\mathcal{R}_{\lambda}(5 / 6)$ land at $-b_{0}$.

Proposition 8. The parameter rays $\mathcal{R}_{\lambda}(1 / 6)$ and $\mathcal{R}_{\lambda}(1 / 3)$ land at the same parameter $b_{0}$ satisfying $b_{0}^{2}=4(\lambda-1)$. The parameter rays $\mathcal{R}_{\lambda}(2 / 3)$ and $\mathcal{R}_{\lambda}(5 / 6)$ land at $-b_{0}$. 


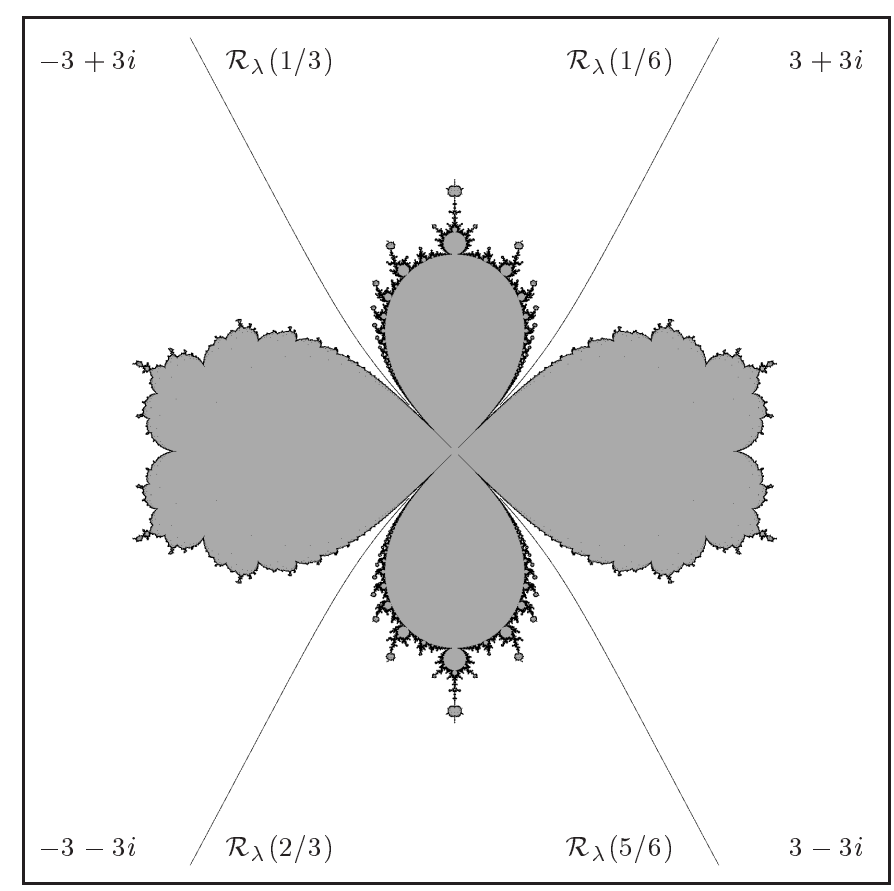

Fig. 6. The parameter space for $\lambda=1$. The four rays $\mathcal{R}_{\lambda}(1 / 6), \mathcal{R}_{\lambda}(1 / 3), \mathcal{R}_{\lambda}(2 / 3)$ and $\mathcal{R}_{\lambda}(5 / 6)$ land at 0.

Remark. When $\lambda=1$, we have $b_{0}=0$ and the four rays land at 0 (see Figure 6 ).

Proof. In the case $\lambda \neq 1$, we will show that for any parameter $b_{0}$ contained in the accumulation set of the ray $\mathcal{R}_{\lambda}(1 / 6), f_{b_{0}}$ has a parabolic fixed point with multiplier 1 . The set of such parameters is discrete - in fact $b_{0}^{2}=4(\lambda-1)$. Since the accumulation set of any ray is connected, this will prove that the ray $\mathcal{R}_{\lambda}(1 / 6)$ lands. A similar argument shows that the rays $\mathcal{R}_{\lambda}(1 / 3), \mathcal{R}_{\lambda}(2 / 3)$ and $\mathcal{R}_{\lambda}(5 / 6)$ land at $b_{0}$ or $-b_{0}$. We will then have to show that the rays $\mathcal{R}_{\lambda}(1 / 6)$ and $\mathcal{R}_{\lambda}(1 / 3)$ land at the same parameter.

In the case $\lambda=1$, we will show that the only parameter in the accumulation set of the rays $\mathcal{R}_{\lambda}(1 / 6), \mathcal{R}_{\lambda}(1 / 3), \mathcal{R}_{\lambda}(2 / 3)$ and $\mathcal{R}_{\lambda}(5 / 6)$ is $b_{0}=0$. This will conclude the proof of the proposition.

Lemma 4. For any parameter $b_{0}$ contained in the accumulation set of the ray $\mathcal{R}_{\lambda}(1 / 6), \mathcal{R}_{\lambda}(1 / 3), \mathcal{R}_{\lambda}(2 / 3)$ or $\mathcal{R}_{\lambda}(5 / 6)$, the polynomial $f_{b_{0}}$ has a parabolic fixed point with multiplier 1 .

Proof. Let us prove this lemma for the ray $\mathcal{R}_{\lambda}(1 / 6)$. We will proceed by contradiction. Assume that $f_{b_{0}}$ has no parabolic fixed point with multiplier 1. Since $b_{0} \in M_{\lambda}$, the dynamical ray $R_{b_{0}}(1 / 2)$ does not bifurcate. It is a fixed dynamical ray. Hence, it lands at a fixed point $\alpha$, which is either repelling, or parabolic with multiplier 1 . By hypothesis on $b_{0}$, the second case is not possible. 
We claim that for $b$ sufficiently close to $b_{0}$, the ray $R_{b}(1 / 2)$ still lands on a repelling fixed point of $f_{b}$. The proof is classical and can be found in the Orsay Notes [DH1].

Thus, for any $b \in \mathcal{U}_{1}$, the ray $R_{b}(1 / 2)$ does not bifurcate on a critical point. In particular, the dynamical ray $R_{b}(1 / 6)$ cannot contain the co-critical point. But this precisely shows that the parameter ray $\mathcal{R}_{\lambda}(1 / 6)$ omits the neighborhood $\mathcal{U}_{1}$ of $b_{0}$ which gives the contradiction.

The fixed points of the polynomial $f_{b}$ are 0 and the roots of the equation $\lambda-1+b z+z^{2}=0$. If $\lambda \neq 1$, there is a multiple root (i.e., a parabolic fixed point with multiplier 1 ) if and only if the discriminant is zero: $b^{2}-4(\lambda-1)=0$. Hence, when $\lambda \neq 1$, we see that the parameter rays $\mathcal{R}_{\lambda}(1 / 6), \mathcal{R}_{\lambda}(1 / 3), \mathcal{R}_{\lambda}(2 / 3)$ and $\mathcal{R}_{\lambda}(5 / 6)$ can only accumulate on $b_{0}$ or $-b_{0}$, where $b_{0}^{2}=4(\lambda-1)$. Since the accumulation set of a ray is connected, we have proved that those rays land at $b_{0}$ or $-b_{0}$.

When $\lambda=1$, the origin is a persistently parabolic fixed point with multiplier 1. Hence, to be able to conclude that the parameter rays land, we must improve our lemma. The following lemma completes the proof of the proposition in the case $\lambda=1$.

Lemma 5. When $\lambda=1$ the parameter rays $\mathcal{R}_{\lambda}(1 / 6), \mathcal{R}_{\lambda}(1 / 3), \mathcal{R}_{\lambda}(2 / 3)$ and $\mathcal{R}_{\lambda}(5 / 6)$ land at $b_{0}=0$.

Proof. Let us prove this lemma for the parameter ray $\mathcal{R}_{\lambda}(1 / 6)$. The proof is essentially the same as in lemma 4 . We proceed by contradiction, assuming that the parameter ray $\mathcal{R}_{\lambda}(1 / 6)$ accumulates on $b_{0} \neq 0$.

On the one hand, the dynamical ray $R_{b_{0}}(1 / 2)$ cannot land at a repelling fixed point, since otherwise there would be a neighborhood $\mathcal{U}_{1}$ of $b_{0}$ in which the dynamical ray $R_{b}(1 / 2)$ would not bifurcate (as in lemma 4 ).

On the other hand, if the dynamical ray $R_{b_{0}}(1 / 2)$ were landing at a parabolic fixed point with multiplier 1 (i.e., the fixed point 0 ) then we could still show that there exists a neighborhood $\mathcal{U}_{1}$ in which the dynamical ray $R_{b}(1 / 2)$ would not bifurcate. The idea of the proof is the following.

Since $b_{0} \neq 0$, the parabolic fixed point 0 is simple, i.e., $f_{b_{0}}^{\prime \prime}(0) \neq 0$. We will show that we can follow continuously a repelling petal $\mathcal{P}_{\text {rep }}(b)$ in a neighborhood $\mathcal{U}_{0}$ of $b_{0}$. On this repelling petal, the inverse branches $f_{b}^{-1}: \mathcal{P}_{\text {rep }}(b) \rightarrow \mathcal{P}_{\text {rep }}(b)$ are well defined and iterates of this inverse branches converge to 0 . We will also show that the dynamical ray $R_{b_{0}}(1 / 2)$ enters the repelling petal $\mathcal{P}_{r e p}\left(b_{0}\right)$. Consequently, there exists a neighborhood $\mathcal{U}_{1}$ of $b_{0}$ such that for any $b \in \mathcal{U}_{1}$, the dynamical ray $R_{b}(1 / 2)$ enters the repelling petal $\mathcal{P}_{r e p}(b)$, and thus land at the parabolic fixed point 0 .

Let us fill in the details. Since we assume $b_{0} \neq 0$, there exists a neighborhood $\mathcal{U}_{0}$ of $b_{0}$ and a radius $\varepsilon>0$ such that for any $b \in \mathcal{U}_{0}, f_{b}$ restricts to an isomorphism between the disk $V(b)$ centered at 0 with radius $\varepsilon /|b|$ and $f_{b}(V(b))$. Now, observe that the change of coordinates $z \mapsto Z=-1 / b z$ conjugates $f_{b}: V(b) \rightarrow f_{b}(V(b))$ to an isomorphism $F_{b}: \widehat{V} \rightarrow F_{b}(\widehat{V})$, where

$$
\widehat{V}=\left\{Z \in \mathbb{P}^{1}|1 / \varepsilon<| Z \mid\right\} \quad \text { and } \quad F_{b}(Z)=Z+1+\mathcal{O}\left(\frac{1}{|Z|}\right) .
$$




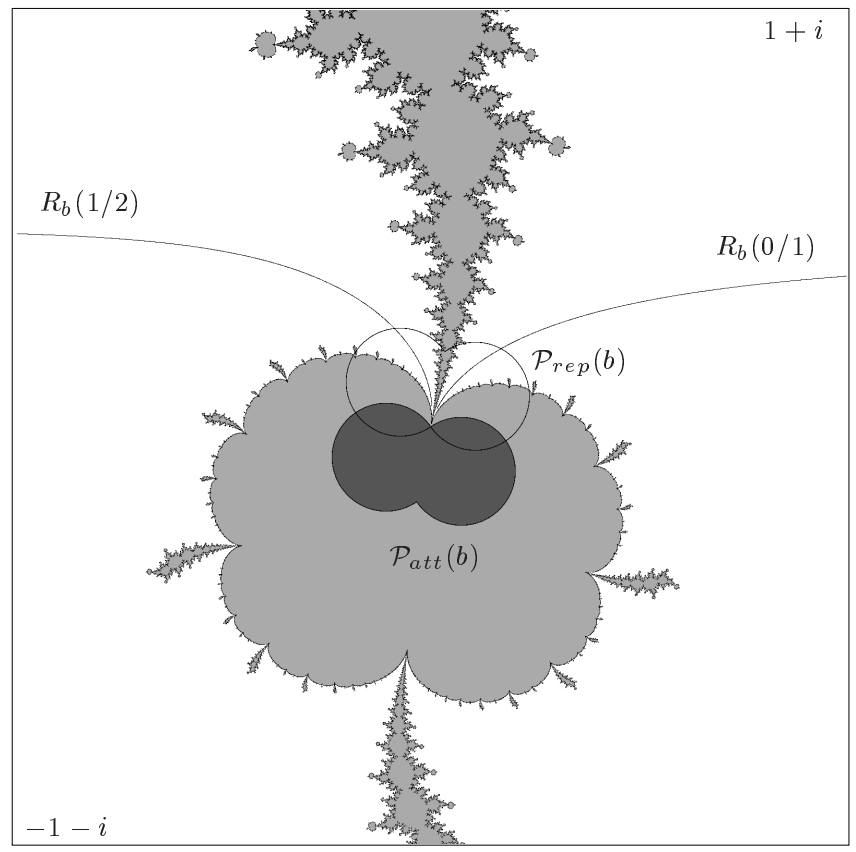

Fig. 7. An attracting petal $\mathcal{P}_{a t t}(b)$ and a repelling petal $\mathcal{P}_{\text {rep }}(b)$. The attracting petal $\mathcal{P}_{a t t}(b)$ is contained in $K\left(f_{b}\right)$ and the ray $R_{b}(1 / 2)$ eventually enters and stays in $\mathcal{P}_{\text {rep }}(b)$.

Let us choose $\varepsilon$ sufficiently small, so that $\left|F_{b}(Z)-Z-1\right|<\sqrt{2} / 2$ for any $b \in \mathcal{U}_{0}$ and any $Z \in \widehat{V}$. Then, denote by $\widehat{\mathcal{P}}_{\text {att }}$ and $\widehat{\mathcal{P}}_{\text {rep }}$ the sectors

$$
\widehat{\mathcal{P}}_{\text {att }}=\{Z \in \mathbb{C}|\sqrt{2} / \varepsilon-\operatorname{Re}(Z)<| \operatorname{Im}(Z) \mid\},
$$

and

$$
\widehat{\mathcal{P}}_{\text {rep }}=\{Z \in \mathbb{C}|\sqrt{2} / \varepsilon+\operatorname{Re}(Z)<| \operatorname{Im}(Z) \mid\} .
$$

Besides, denote by $\mathcal{P}_{\text {att }}(b)$ and $\mathcal{P}_{\text {rep }}(b)$ the sets

$$
\mathcal{P}_{\text {att }}(b)=\left\{z \in \mathbb{C}^{*} \mid-1 / b z \in \widehat{\mathcal{P}}_{\text {att }}\right\},
$$

and

$$
\mathcal{P}_{\text {rep }}(b)=\left\{z \in \mathbb{C}^{*} \mid-1 / b z \in \widehat{\mathcal{P}}_{\text {rep }}\right\} .
$$

The set $\mathcal{P}_{\text {att }}(b)$ is called an attracting petal and the set $\mathcal{P}_{\text {rep }}(b)$ is called a repelling petal (see Figure 7 ). One can easily check that the assumptions on $\varepsilon$ implies that for any $b \in \mathcal{U}_{0}$, we have

(1) $f_{b}\left(\mathcal{P}_{\text {att }}(b)\right) \subset \mathcal{P}_{\text {att }}(b)$;

(2) $f_{b}^{\circ n}$ converges uniformly on compact subsets of $\mathcal{P}_{a t t}(b)$ to 0 ;

(3) there exists an inverse branch $f_{b}^{-1}: \mathcal{P}_{\text {rep }}(b) \rightarrow \mathcal{P}_{\text {rep }}(b)$;

(4) $\left[f_{b}^{-1}\right]^{\text {on }}$ converges uniformly on compact subsets of $\mathcal{P}_{\text {rep }}(b)$ to 0 . 
Let us express the ray $R_{b_{0}}(1 / 2)$ as a countable union of segments

$$
S_{j}=\varphi_{b_{0}}^{-1}\left\{-e^{t} \mid 3^{j} \leq t \leq 3^{j+1}\right\}, \quad j \in \mathbb{Z},
$$

so that $f_{b_{0}}\left(S_{j}\right)=S_{j+1}$. Clearly, we wee that $\mathcal{P}_{a t t}\left(b_{0}\right)$ is contained in the filled-in Julia set $K\left(f_{b_{0}}\right)$. Thus, $R_{b_{0}}(1 / 2)$ does not intersect $\mathcal{P}_{a t t}\left(b_{0}\right)$. Since we assumed that the ray $R_{b_{0}}(1 / 2)$ lands at 0 , there exists an integer $j_{0}$ such that $S_{j_{0}}$ is contained in $\mathcal{P}_{\text {rep }}\left(b_{0}\right)$. Again, by shrinking $\mathcal{U}_{0}$ if necessary, we may assume that $\mathcal{U}_{0} \subset\left\{b \in \mathbb{C} \mid G(b)<3^{j_{0}}\right\}$. This condition implies that for any $b \in \mathcal{U}_{0}$, the ray $R_{b}(1 / 2)$ is defined up to potential at least $3^{j_{0}}$, and

$$
S_{j}(b)=\varphi_{b}^{-1}\left\{-e^{t} \mid 3^{j} \leq t \leq 3^{j+1}\right\}, \quad j \geq j_{0}
$$

is well defined. Finally, since $\left\{(z, b) \mid b \in \mathcal{U}_{0}, z \in \mathcal{P}_{\text {rep }}(b)\right\}$ is open and since $\varphi_{b}^{-1}$ depends continuously (even analytically) on $b$, we see that there exists a neighborhood $\mathcal{U}_{1} \subset \mathcal{U}_{0}$ of $b_{0}$, such that for any $b \in \mathcal{U}_{1}$ the segment $S_{j_{0}}(b)$ is contained in $\mathcal{P}_{\text {rep }}(b)$. Hence, $S_{j_{0}+k}(b)=\left[f_{b}^{-1}\right]^{\circ k}\left(S_{j_{0}}(b)\right)$ is well defined for any $k \geq 0$, and the ray $R_{b}(1 / 2)$ lands at 0 . However, this implies that the parameter ray $\mathcal{R}(1 / 6)$ does not intersect $\mathcal{U}_{1}$.

We still need to prove that when $\lambda \neq 1$, the parameter rays $\mathcal{R}_{\lambda}(1 / 6)$ and $\mathcal{R}_{\lambda}(1 / 3)$ land at the same parameter. Remember that we defined the wake $\mathcal{W}_{0}$ as the connected component of

$$
\mathbb{C} \backslash \overline{\mathcal{R}_{\lambda}(1 / 6)} \cup \overline{\mathcal{R}_{\lambda}(1 / 3)} \cup \overline{\mathcal{R}_{\lambda}(2 / 3)} \cup \overline{\mathcal{R}_{\lambda}(5 / 6)}
$$

that contains the parameter ray $\mathcal{R}_{\lambda}(1 / 4)$.

Let us call $b_{0}$ the landing point of the parameter ray $\mathcal{R}_{\lambda}(1 / 6)$. We will use the fact that the connectedness locus $M_{\lambda}$ is symmetric with respect to 0 (remember that $f_{b}$ and $f_{-b}$ are conjugate by $\left.z \mapsto-z\right)$. The symmetry of $M_{\lambda}$ shows that two of the four rays $\mathcal{R}_{\lambda}(1 / 6), \mathcal{R}_{\lambda}(1 / 3), \mathcal{R}_{\lambda}(2 / 3)$ or $\mathcal{R}_{\lambda}(5 / 6)$ land at $b_{0}$ and the other two land at $-b_{0}$. Moreover, the parameter rays $\mathcal{R}_{\lambda}(1 / 6)$ and $\mathcal{R}_{\lambda}(2 / 3)$ are symmetric, so that $\mathcal{R}_{\lambda}(2 / 3)$ cannot land at $b_{0}\left(\neq-b_{0}\right)$. Hence, if the parameter ray $\mathcal{R}_{\lambda}(1 / 3)$ were not landing at $b_{0}$, then the ray $\mathcal{R}_{\lambda}(5 / 6)$ would. In that case, the wake $\mathcal{W}_{0}$ would contain the parameter $b=0$ (see Figure 8 ). We will get a contradiction by proving that for any parameter $b \in \mathcal{W}_{0}$, the dynamical rays $R_{b}(0 / 1)$ and $R_{b}(1 / 2)$ land at the same point, whereas this is not the case for $b=0$.

Lemma 6. For any parameter $b \in \mathcal{W}_{0}$, the two dynamical rays $R_{b}(0 / 1)$ and $R_{b}(1 / 2)$ do not bifurcate.

Remark. This lemma and the following one are in fact true as soon as $b$ does not belong to one of the parameter rays $\mathcal{R}_{\lambda}(1 / 6), \mathcal{R}_{\lambda}(1 / 3), \mathcal{R}_{\lambda}(2 / 3)$ or $\mathcal{R}_{\lambda}(5 / 6)$.

Proof. If $b \notin \mathcal{R}_{\lambda}(1 / 3) \cup \mathcal{R}_{\lambda}(2 / 3)$, the dynamical ray $R_{b}(0 / 1)$ does not bifurcate. Indeed, if $R_{b}(0 / 1)$ were bifurcating, it would bifurcate on a preimage of the escaping critical point $\omega_{2}$, i.e., there would be a non-negative $n$, such that $f_{b}^{-n}\left(\omega_{2}\right)$ belongs to the ray $R_{b}(0 / 1)$. Since this is a fixed ray, $\omega_{2}$ would belong to the ray $R_{b}(0 / 1)$ and consequently $\omega_{2}^{\prime}$ would lie on either $R_{b}(1 / 3)$ or $R_{b}(2 / 3)$ which contradicts that $b \notin \mathcal{R}_{\lambda}(1 / 3) \cup \mathcal{R}_{\lambda}(2 / 3)$. A similar argument shows that if $b \notin \mathcal{R}_{\lambda}(1 / 6) \cup \mathcal{R}_{\lambda}(5 / 6)$, the dynamical ray $R_{b}(1 / 2)$ does not bifurcate and also lands at a fixed point which is either repelling or parabolic with multiplier 1. 


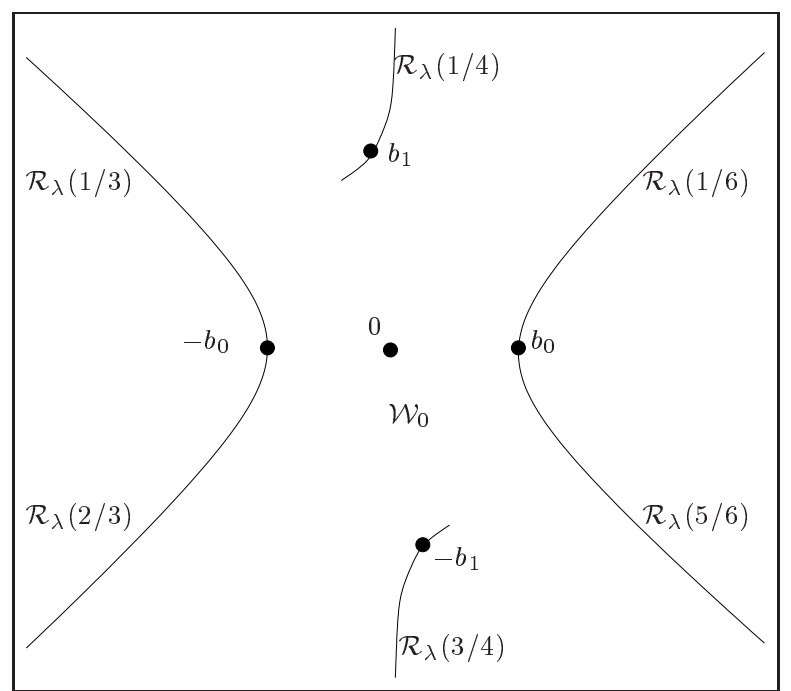

Fig. 8. If the parameter rays $\mathcal{R}_{\lambda}(1 / 6)$ and $\mathcal{R}_{\lambda}(1 / 3)$ were not landing at the same parameter, the wake $\mathcal{W}_{0}$ would contain the parameter $b=0$.

Lemma 7. If $\lambda \neq 1$, then given any $b \in \mathcal{W}_{0}$, the rays $R_{b}(0 / 1)$ and $R_{b}(1 / 2)$ both land at the same repelling fixed point $\beta(b) \neq 0$. If $\lambda=1$, then given any $b \in \mathcal{W}_{0}$, the rays $R_{b}(0 / 1)$ and $R_{b}(1 / 2)$ both land at the parabolic fixed point $\beta(b)=0$.

Proof. To prove this lemma, we will use an idea due to Peter Haïssinsky which has been explained to us by Carsten Petersen. We have seen that in the domain $\mathcal{W}_{0}$, the dynamical rays $R_{b}(0 / 1)$ and $R_{b}(1 / 2)$ do not bifurcate. It follows that the set $X=R_{b}(0 / 1) \cup R_{b}(1 / 2)$ moves holomorphically with respect to the parameter $b$. Hence, the $\lambda$-Lemma by Mañe, Sad and Sullivan [MSS] shows that the closure of $X$ in $\mathbb{P}^{1}$ moves holomorphically. In particular, if for some parameter $b_{1} \in \mathcal{W}_{0}$ the two dynamical rays $R_{b}(0 / 1)$ and $R_{b}(1 / 2)$ land at the same fixed point, they do so everywhere in $\mathcal{W}_{0}$, i.e., there exists a holomorphic function $\beta(b)$ such that $\beta(b)$ is a fixed point of $f_{b}$ and is the landing point of the two rays $R_{b}(0 / 1)$ and $R_{b}(1 / 2)$. Besides, the multiplier at $\beta(b)$ is a univalent function, that takes values in $\mathbb{C} \backslash \mathbb{D}$. Hence, either the multiplier is constantly equal to 1 (which corresponds to a persistently parabolic landing point) or it takes values in $\mathbb{C} \backslash \overline{\mathbb{D}}$ (and the landing point remains repelling in all $\mathcal{W}_{0}$ ).

Thus, we just need to show that there is a parameter $b \in \mathcal{W}_{0}$ for which the two rays $\mathcal{R}_{b}(0 / 1)$ and $R_{b}(1 / 2)$ land at a common fixed point, and that this point is repelling when $\lambda \neq 1$, whereas it is parabolic with multiplier 1 when $\lambda=1$. This is precisely given by proposition 6 for the parameter $b_{1}=\Phi_{\lambda}^{-1}\left(e^{1 / 3+2 i \pi / 4}\right)$.

To conclude the proof of the proposition, it is enough to see that when $b=0$ and $\lambda \neq 1$, the two dynamical rays $R_{0}(0 / 1)$ and $R_{0}(1 / 2)$ cannot land at the same point. The polynomial $f_{0}(z)=\lambda z+z^{3}$ is an odd polynomial. Thus, the filled-in Julia set is symmetric with respect to the origin. In particular, the dynamical rays $R_{0}(0 / 1)$ and $R_{0}(1 / 2)$ are symmetric. Thus, if they land (in fact, 
the two critical orbits are symmetric, the Julia set is connected, and the rays land) the landing points are symmetric with respect to the origin. However, the origin cannot be the landing point of those rays because it is indifferent with multiplier $\lambda \neq 1$. Hence, the two dynamical rays $R_{0}(0 / 1)$ and $R_{0}(1 / 2)$ land at two symmetric, distinct fixed points.

We have proved that in the wake $\mathcal{W}_{0}$, the two dynamical rays $R_{b}(0 / 1)$ and $R_{b}(1 / 2)$ both land at a common fixed point $\beta(b)$ which depends holomorphically on $b$. If $\lambda=1$, we have seen that $\beta(b)=0$ is a double fixed point, and the cubic polynomial $f_{b}$ has only one other fixed point: $\alpha(b)=-b$. If $\lambda \neq 1$, the map $f_{b}$ has three distinct fixed points: $0, \beta(b)$ and $\alpha(b)=-b-\beta(b)$.

Definition 12. For any $b \in \mathcal{W}_{0}$, we call $\beta(b)$ the landing point of the dynamical rays $R_{b}(0 / 1)$ and $R_{b}(1 / 2)$, and we call $\alpha(b)=-b-\beta(b)$ the fixed point of $f_{b}$ which is neither 0 nor $\beta(b)$.

Remark. Since the function $\beta$ is holomorphic in $\mathcal{W}_{0}$, the function $\alpha$ is also holomorphic in $\mathcal{W}_{0}$. In fact, since $\mathcal{W}_{0}$ is simply connected and does not contain the parameters $\pm b_{0}$, it is clear that the three fixed points of $f_{b}$ depend holomorphically on $b$ in $\mathcal{W}_{0}$, without using the fact that $\beta(b)$ is the landing parameter of the rays $R_{b}(0 / 1)$ and $R_{b}(1 / 2)$.

\section{Dynamics of $f_{b}$ in the wake $\mathcal{W}_{0}$.}

We will now improve our description of the dynamical behaviour of the polynomial $f_{b}$, when $b \in \mathcal{W}_{0}$ (see Figure 9).

Proposition 9. For any $b \in \mathcal{W}_{0}$, the dynamics of the map $f_{b}$ is as follows:

1. the two critical points of $f_{b}$ are distinct and there exist two holomorphic functions $\omega_{1}(b)$ and $\omega_{2}(b)$ defined in $\mathcal{W}_{0}$, such that for any $b \in \mathcal{W}_{0}, \omega_{1}(b)$ and $\omega_{2}(b)$ are the two critical points of $f_{b}, \omega_{2}(b)$ being the escaping critical point whenever $b \in \mathcal{W}_{0} \backslash M_{\lambda}$; the co-critical points are $\omega_{i}^{\prime}(b)=-b-2 \omega_{i}(b)$;

2. the dynamical rays $R_{b}(1 / 6)$ and $R_{b}(1 / 3)$ do not bifurcate and both land at a preimage $\beta_{1}(b) \neq \beta(b)$ of $\beta(b)$; the rays $R_{b}(2 / 3)$ and $R_{b}(5 / 6)$ do not bifurcate and land at the other preimage $\beta_{2}(b) \notin\left\{\beta(b), \beta_{1}(b)\right\}$; we define $V_{i}$ to be the connected component of $\mathbb{C} \backslash \overline{R_{b}(0 / 1)} \cup \overline{R_{b}(1 / 2)}$ that contains $\beta_{i}(b)$;

3. each of the four connected components of $\mathbb{C} \backslash \bigcup_{\{\theta \mid 6 \theta \in \mathbb{Z}\}} \overline{R_{b}(\theta)}$ contains exactly one of the four points $\omega_{1}(b), \omega_{2}(b), \omega_{1}^{\prime}(b)$ or $\omega_{2}^{\prime}(b)$; we call $U_{i}, i=1,2$, the one containing $\omega_{i}(b)$ and $U_{i}^{\prime}, i=1,2$, the one containing $\omega_{i}^{\prime}(b)$;

4. the map $f_{b}: U_{i}^{\prime} \rightarrow V_{i}, i=1,2$, is an isomorphism and the map $f_{b}: U_{i} \rightarrow V_{i}$, $i=1,2$, is a ramified covering of degree 2 ramified at $\omega_{i}(b)$.

Proof. We will first show that we can follow the two critical points holomorphically when $b \in \mathcal{W}_{0}$.

Lemma 8. For any $b \in \mathcal{W}_{0}$, the two critical points of $f_{b}$ are distinct. Moreover, there exist two holomorphic functions $\omega_{1}(b)$ and $\omega_{2}(b)$ defined in $\mathcal{W}_{0}$, such that for any $b \in \mathcal{W}_{0}, \omega_{1}(b)$ and $\omega_{2}(b)$ are the two critical points of $f_{b}$. 


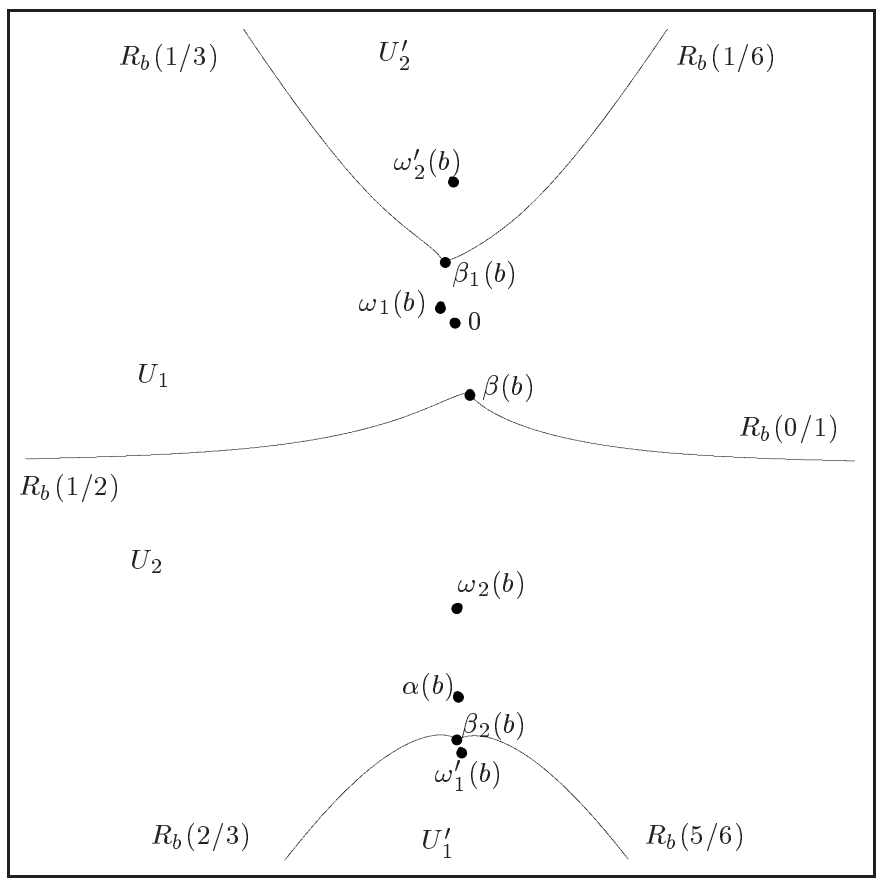

Fig. 9. The dynamical picture of the polynomial $f_{b}$ when the parameter $b$ belongs to $\mathcal{W}_{0}$.

Proof. When the two critical points of $f_{b}$ are distinct, i.e., $b^{2} \neq 3 \lambda$, we can locally follow them. Since $\mathcal{W}_{0}$ is simply connected, the proof of the lemma will be completed once we have proved that for any $b \in \mathcal{W}_{0}$, the two critical points of $f_{b}$ are distinct.

We will proceed by contradiction and assume that for some parameter $b \in \mathcal{W}_{0}$, the polynomial $f_{b}$ has a unique critical point $\omega$. The polynomial $f_{b}$ is then conjugate by the affine change of coordinate $z \mapsto w=z-\omega$ to a polynomial of the form $w \mapsto w^{3}+c$. The Julia set of such a polynomial is invariant under the rotation $w \mapsto e^{2 i \pi / 3} w$. This shows that the Julia set of $f_{b}$ is invariant under the rotation of angle $1 / 3$ around $\omega$. In particular, the dynamical ray $R_{b}(1 / 3)$ (respectively $R_{b}(2 / 3)$ ) is the image of the dynamical ray $R_{b}(0 / 1)$ by the rotation of angle $1 / 3$ (respectively 2/3) of center $\omega$ (see Figure 10). For the same reason, the dynamical ray $R_{b}(5 / 6)$ (respectively $R_{b}(1 / 6)$ ) is obtained from $R_{b}(1 / 2)$ by rotating with angle $1 / 3$ (respectively $2 / 3$ ) around $\omega$. We will show that the dynamical rays $R_{b}(0 / 1)$ and $R_{b}(1 / 2)$ cannot land at the same point $\beta(b)$.

Indeed, when $b \in \mathcal{W}_{0}$, the two dynamical rays $R_{b}(0 / 1)$ and $R_{b}(1 / 2)$ land at $\beta(b)$. By rotating with angle $1 / 3$, we see that the two rays $R_{b}(1 / 3)$ and $R_{b}(5 / 6)$ land at $e^{2 i \pi / 3} \beta(b)$. Since those two rays are separated by the curve $\{\beta(b)\} \cup$ $R_{b}(0 / 1) \cup R_{b}(1 / 2)$, they can only meet at $\beta(b)$. Hence, $\beta(b)=e^{2 i \pi / 3}(\beta(b)-\omega)+$ $\omega=\omega$. But this would imply that $\omega$ is a super-attracting fixed point, and no ray could land at $\omega$. This gives the contradiction. 


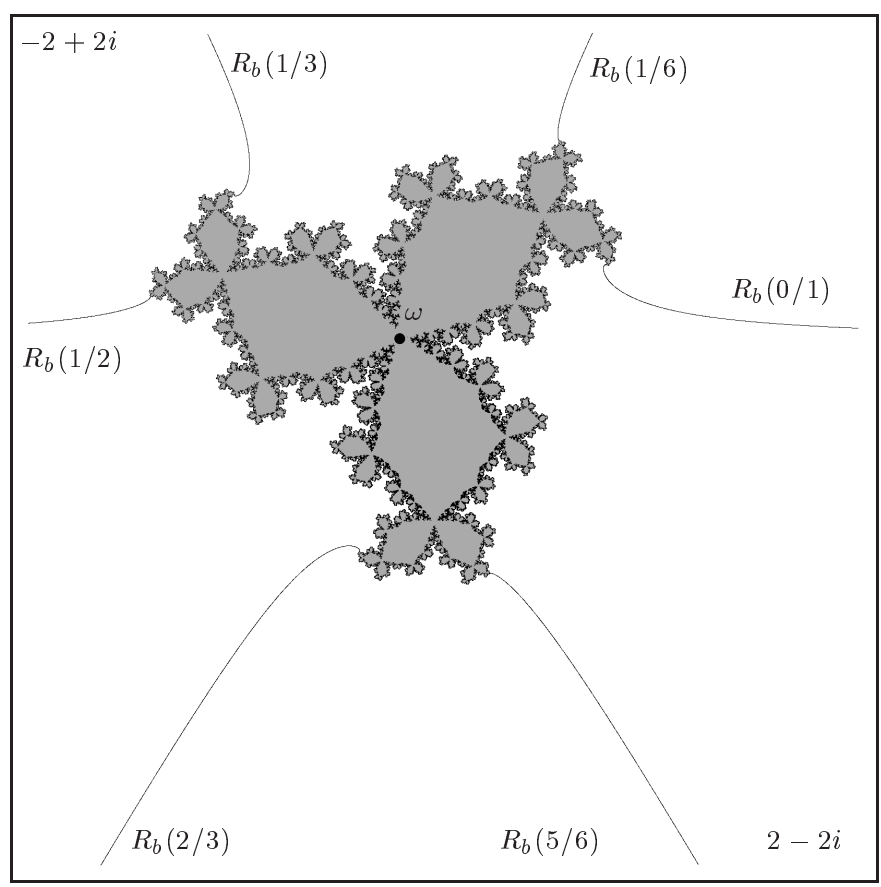

Fig. 10. The Julia set of $f_{b}$ for $b^{2}=3 \lambda$. There is a unique critical point $\omega$ and the Julia set is invariant by rotation of angle $1 / 3$ around $\omega$.

Then, it is not difficult to check that the co-critical points $\omega_{i}^{\prime}(b)$ are defined by $\omega_{i}^{\prime}(b)=-b-2 \omega_{i}(b), i=1,2$. We still have a choice on which critical point will be labelled $\omega_{1}$ and which one will be labelled $\omega_{2}$. To complete the proof of (1), we need to prove that we can choose $\omega_{2}$ such that $\omega_{2}(b)$ is the escaping critical point of $f_{b}$ for any $b \in \mathcal{W}_{0} \backslash M_{\lambda}$. This will be done later and we will now focus on the proof of (2).

Lemma 9. For any $b \in \mathcal{W}_{0}$ the dynamical rays $R_{b}(1 / 6)$ and $R_{b}(1 / 3)$ do not bifurcate. They both land at a preimage $\beta_{1}(b) \in f_{b}^{-1}\{\beta(b)\} \backslash\{\beta(b)\}$ of $\beta(b)$. The rays $R_{b}(2 / 3)$ and $R_{b}(5 / 6)$ do not bifurcate and land at the other preimage $\beta_{2}(b) \in f_{b}^{-1}\{\beta(b)\} \backslash\left\{\beta(b), \beta_{1}(b)\right\}$.

Proof. Let us assume that $R_{b}(1 / 6)$ bifurcates for some parameter $b \in \mathcal{W}_{0}$. Then it bifurcates on a preimage of the escaping critical point $\omega_{2}$, and one of its forward image bifurcates on $\omega_{2}$. Since $f_{b}\left(R_{b}(1 / 6)\right)=R_{b}(1 / 2)$ is fixed, this means that $\omega_{2}$ belongs to the ray $R_{b}(1 / 6)$ or to the ray $R_{b}(1 / 2)$. On the one hand, the latter case is not possible since the ray $R_{b}(1 / 2)$ does not bifurcate. On the other hand, since $b \in \mathcal{W}_{0}$, the escaping co-critical point $\omega_{2}^{\prime}$ belongs to a dynamical ray $R_{b}(\theta)$, with $\theta \in] 1 / 6,1 / 3\left[\right.$. Hence, the rays bifurcating on $\omega_{2}$ have angle $\left.\theta-1 / 3 \in\right]-1 / 6,0[$ and $\theta+1 / 3 \in] 1 / 2,2 / 3\left[\right.$. Thus, the ray $R_{b}(1 / 6)$ cannot bifurcate on $\omega_{2}$.

A similar argument shows that the rays $R_{b}(1 / 3), R_{b}(2 / 3)$ and $R_{b}(5 / 6)$ do not bifurcate.

To complete the proof of the lemma, it is enough to prove that $\beta(b)$ has three distinct preimages: $\beta(b), \beta_{1}(b)$ and $\beta_{2}(b)$. In other words, we need to show that 
$\beta(b)$ is not a critical value of $f_{b}$. Indeed, we can then argue that since $f_{b}$ is a local isomorphism in a neighborhood of $\beta_{i}(b)$ and since the rays $R_{b}(0 / 1)$ and $R_{b}(1 / 2)$ land at $\beta(b)$, two of the rays $R_{b}(1 / 6), R_{b}(1 / 3), R_{b}(2 / 3)$ and $R_{b}(5 / 6)$ land at $\beta_{1}(b)$ and two of them land at $\beta_{2}(b)$. The only possibility is that $R_{b}(1 / 6)$ and $R_{b}(1 / 3)$ land at the same preimage, let us say $\beta_{1}(b)$, and the rays $R_{b}(2 / 3)$ and $R_{b}(5 / 6)$ land at the other preimage $\beta(b)$.

To see that $\beta(b)$ is not a critical value of $f_{b}$, we will proceed by contradiction. Hence, we assume that for some parameter $b \in \mathcal{W}_{0}$, one critical point $\omega$ is mapped by $f_{b}$ to $\beta(b)$. Then, since $\beta(b)$ is either repelling or parabolic with multiplier 1 , we see that $\omega \neq \beta(b)$. Besides, we have seen that the two critical points of $f_{b}$ are distinct. Hence, in a neighborhood of $\omega$, the map $f_{b}$ is a two-to-one ramified covering, and the four rays $R_{b}(1 / 6), R_{b}(1 / 3), R_{b}(2 / 3)$ and $R_{b}(5 / 6)$ have to land at $\omega$. But this is not possible since the rays $R_{b}(1 / 6), R_{b}(2 / 3)$ are separated by $R_{b}(0 / 1)$ and $R_{b}(1 / 2)$.

We will now prove (3) using a holomorphic motion argument.

Lemma 10. The set

$$
X_{b}=\left\{\omega_{1}(b), \omega_{2}(b), \omega_{1}^{\prime}(b), \omega_{2}^{\prime}(b)\right\} \cup\left(\bigcup_{\{\theta \mid 6 \theta \in \mathbb{Z}\}} \overline{R_{b}(\theta)}\right)
$$

undergoes a holomorphic motion as $b$ moves in $\mathcal{W}_{0}$.

Proof. The functions $\omega_{i}(b)$ and $\omega_{i}^{\prime}(b), i=1,2$, are holomorphic when $b \in \mathcal{W}_{0}$. Besides, we have seen that the dynamical rays $R_{b}(\theta), 6 \theta \in \mathbb{Z}$, do not bifurcate when $b \in \mathcal{W}_{0}$, and thus, move holomorphically when $b \in \mathcal{W}_{0}$. To prove the lemma, we need to prove the injectivity condition of holomorphic motions. Since we already know that the critical points are distinct, we only need to show that for any $b \in \mathcal{W}_{0}$, the critical points and co-critical points cannot belong to any of the rays $\overline{R_{b}(\theta)}, 6 \theta \in \mathbb{Z}$. But this is clear since otherwise, one of those rays would have to bifurcate on a critical point.

The dynamical picture for the polynomial $f_{b_{1}}$ has been studied in section 3 , and it is not difficult to check that each connected component of

$$
\mathbb{C} \backslash \bigcup_{\{\theta \mid 6 \theta \in \mathbb{Z}\}} \overline{\mathcal{R}_{b_{1}}(\theta)}
$$

contains exactly one of the four points $\omega_{1}\left(b_{1}\right), \omega_{2}\left(b_{1}\right), \omega_{1}^{\prime}\left(b_{1}\right)$ or $\omega_{2}^{\prime}\left(b_{1}\right)$. None of the four points are contained in the set $\bigcup_{\{\theta \mid 6 \theta \in \mathbb{Z}\}} \overline{\mathcal{R}_{b_{1}}(\theta)}$ for any $b \in \mathcal{W}_{0}$. Since the four points and $\bigcup_{\{\theta \mid 6 \theta \in \mathbb{Z}\}} \overline{\mathcal{R}_{b_{1}}(\theta)}$ moves continuously when $b$ changes and $\mathcal{W}_{0}$ is connected, statement (3) follows.

We can now complete the proof of $(1)$. We choose the functions $\omega_{1}(b)$ and $\omega_{2}(b)$ so that $\omega_{2}\left(b_{1}\right)$ is the escaping critical point of $f_{b_{1}}$. Then, the boundary of $U_{2}^{\prime}\left(b_{1}\right)$ is the union of the two dynamical rays $R_{b_{1}}(1 / 6), R_{b_{1}}(1 / 3)$ and their landing point $\beta_{1}\left(b_{1}\right)$. Using the holomorphic motion, we see that the same property holds for $U_{2}^{\prime}(b)$, i.e., the boundary of $U_{2}^{\prime}(b)$ is the union of the two dynamical rays $R_{b}(1 / 6), R_{b}(1 / 3)$ and their landing point $\beta_{1}(b)$. In particular, the region $U_{2}^{\prime}(b)$ contains the dynamical rays $\left.R_{b}(\theta), \theta \in\right] 1 / 6,1 / 3[$. On the other hand, we 
know that when $b \in \mathcal{W}_{0} \backslash M_{\lambda}$, the escaping co-critical point belongs to one of those rays. Hence, for any $b \in \mathcal{W}_{0} \backslash M_{\lambda}$ the escaping co-critical point belongs to the region $U_{2}^{\prime}(b)$. Thus the escaping co-critical point is $\omega_{2}^{\prime}(b)$ and for any $b \in \mathcal{W}_{0} \backslash M_{\lambda}$, the escaping critical point is $\omega_{2}(b)$.

We finally prove (4). We have called $V_{1}(b)$ and $V_{2}(b)$ the two connected com-

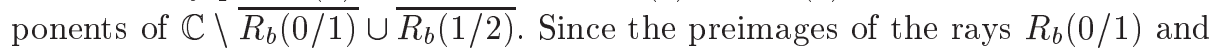
$R_{b}(1 / 2)$ are the rays $R_{b}(\theta), 6 \theta \in \mathbb{Z}$, the connected components of $f_{b}^{-1}\left(V_{i}\right)$, $i=1,2$, are the connected components of $\mathbb{C} \backslash \bigcup_{\{\theta \mid 6 \theta \in \mathbb{Z}\}} \overline{R_{b}(\theta)}$. Let $U$ be one of them. Since the polynomial $f_{b}: \mathbb{C} \rightarrow \mathbb{C}$ is a ramified covering, the restriction of $f_{b}$ to $U$ is a ramified covering onto its image. Since $U$ is simply connected, the Riemann-Hurwitz formula shows that the degree of the restriction of $f_{b}$ to $U$ is $n+1$ where $n$ is the number of critical points of $f_{b}$ in $U$, counted with multiplicity. Hence, to finish the proof of (4), we only need to show that $f_{b}\left(U_{i}\right)=V_{i}$ and $f_{b}\left(U_{i}^{\prime}\right)=V_{i}$ for $i=1,2$.

Lemma 11. For any $b \in \mathcal{W}_{0}$, the component $U_{2}^{\prime}$ contains the two dynamical rays $R_{b}(2 / 9)$ and $R_{b}(5 / 18)$ that both land at a preimage of $\beta_{2}(b)$.

Proof. We have seen previously that for any $b \in \mathcal{W}_{0}$, the region $U_{2}^{\prime}$ contains the dynamical rays $\left.R_{b}(\theta), \theta \in\right] 1 / 6,1 / 3[$. Since $2 / 9 \in] 1 / 6,1 / 3[$ and $5 / 18 \in] 1 / 6,1 / 3[$, the first part of the lemma is proved.

Next, we have seen that $f_{b}$ is an isomorphism between $U_{2}^{\prime}$ and its image. Since $U_{2}^{\prime}$ contains the two dynamical rays $R_{b}(2 / 9)$ and $R_{b}(5 / 18)$, its image contains the two dynamical rays $f_{b}\left(R_{b}(2 / 9)\right)=R_{b}(2 / 3)$ and $f_{b}\left(R_{b}(5 / 18)\right)=R_{b}(5 / 6)$ that both land at $\beta_{2}(b) \in V_{2}$ and the lemma is proved.

Since $f_{b}$ maps the rays $R_{b}(2 / 9)$ and $R_{b}(5 / 18)$ which are in $U_{2}^{\prime}$ to the rays $R_{b}(2 / 3)$ and $\mathcal{R}_{b}(5 / 6)$ which land at $\beta_{2}(b) \in V_{2}$, we see that $f_{b}\left(U_{2}^{\prime}\right)=V_{2}$. Since $\omega_{2}(b)$ and $\omega_{2}^{\prime}(b)$ have the same image, we immediately obtain that $f_{b}\left(U_{2}\right)=V_{2}$. Hence, $f_{b}: U_{2}^{\prime} \rightarrow V_{2}$ is an isomorphism and $f_{b}: U_{2} \rightarrow V_{2}$ is a ramified covering of degree 2 , ramified at $\omega_{2}$. Since the polynomial $f_{b}$ has degree 3 , the component $V_{2}$ has no other preimage, and $f_{b}\left(U_{1}\right)=f_{b}\left(U_{1}^{\prime}\right)=V_{1}$. This finishes the proof of the proposition.

\section{Holomorphic motion of rays.}

In the rest of this article, we will work in the wake $\mathcal{W}_{0}$. We will constantly have to deal with the critical point $\omega_{2}(b), b \in \mathcal{W}_{0}$. Thus, the reader must keep in mind that the function $\omega_{2}$ is a holomorphic function defined throughout all the wake $\mathcal{W}_{0}$, and that for any parameter $b \in \mathcal{W}_{0} \backslash M_{\lambda}$, the point $\omega_{2}(b)$ is the escaping critical point.

Theorem A. For any parameter $b \in \mathcal{W}_{0}$ and for any $\theta \in \Theta$, the dynamical ray $R_{b}(\theta)$ does not bifurcate. We define $X_{b}$ to the set

$$
X_{b}=\bigcup_{\theta \in \Theta} R_{b}(\theta) .
$$

We also define $J_{b}$ to be the set $J_{b}=\overline{X_{b}} \backslash X_{b}$ and $K_{b}$ to be the complement of the unbounded connected component of $\mathbb{C} \backslash J_{b}$. Then, $K_{b}$ is contained in the filled-in 
Julia set $K\left(f_{b}\right)$, its boundary $J_{b}$ is contained in the Julia set $J\left(f_{b}\right)$ and $K_{b}$ is quasi-conformally homeomorphic to the filled-in Julia set $K\left(\lambda z+z^{2}\right)$.

Figure 11 shows the set $K_{b}$ and the set of dynamical rays $X_{b}$ for a parameter $b \in M_{\lambda} \cap \mathcal{W}_{0}$.

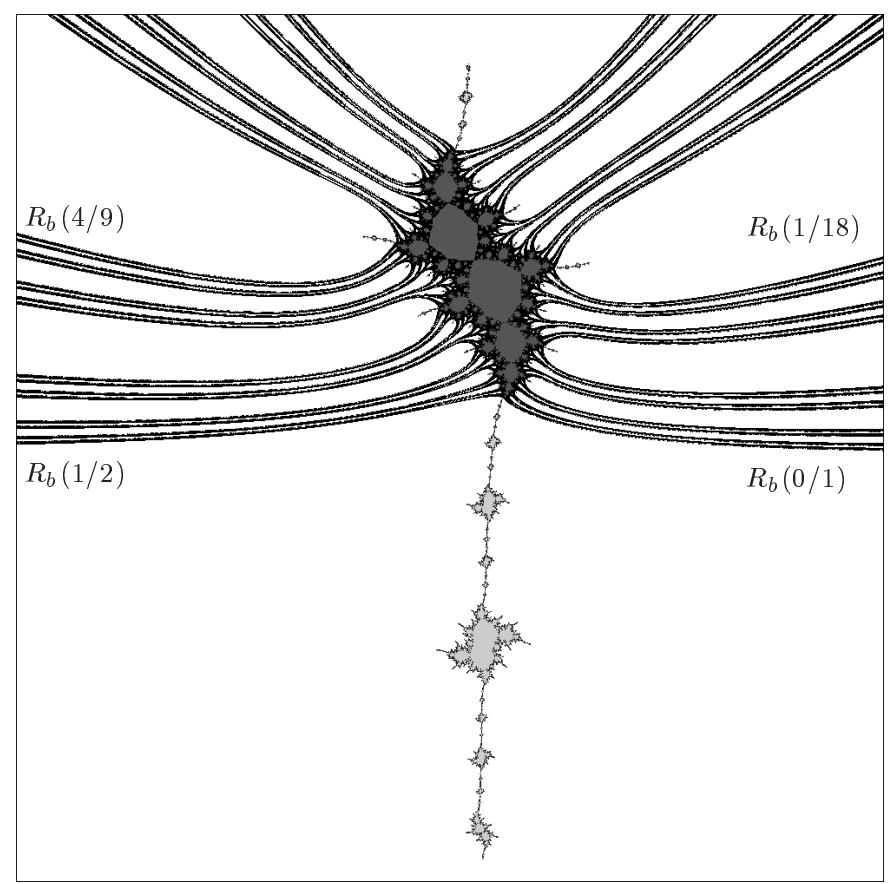

Fig. 11. The set $K_{b}$ and the set of set of dynamical rays $X_{b}$ for a parameter $b \in M_{\lambda} \cap \mathcal{W}_{0}$.

Proof. Let us first prove that for any parameter $b \in \mathcal{W}_{0}$ and any $\theta \in \Theta$, the dynamical ray $R_{b}(\theta)$ does not bifurcate. We will mimic the proof of proposition 7 .

For any $b \in \mathcal{W}_{0}$, we have defined $V_{2}(b)$ to be the connected component of $\mathbb{C} \backslash \overline{R_{b}(0 / 1)} \cup \overline{R_{b}(1 / 2)}$ that contains $\beta_{2}(b)$. Since the two dynamical rays $R_{b}(2 / 3)$ and $R_{b}(5 / 6)$ land at $\beta_{2}(b)$, they are contained in $V_{2}(b)$, and for any $\theta \in[0,1 / 2]$, the dynamical ray $R_{b}(\theta)$ is contained in $\mathbb{C} \backslash V_{2}(b)$. Since for any $\theta \in \Theta$, we have $3^{k} \theta \in[0,1 / 2] \bmod 1$, for any $k \geq 0$, the forward orbit of the ray $R_{b}(\theta)$ is contained in $\mathbb{C} \backslash V_{2}(b)$. Next, for any $b \in \mathcal{W}_{0}$, we claim that the critical point $\omega_{2}(b)$ - which is the escaping critical point when $b \in \mathcal{W}_{0} \backslash M_{\lambda}$ - belongs to the region $V_{2}(b)$. Indeed, we have seen that $\beta(b)$ cannot be a critical value of $f_{b}$. Hence, the set $\left\{\omega_{2}(b), \beta_{2}(b)\right\} \cup R_{b}(0 / 1) \cup R_{b}(1 / 2)$ moves holomorphically when $b \in \mathcal{W}_{0}$. Hence, $\omega_{2}(b)$ and $\beta_{2}(b)$ are always in the same connected component of $\mathbb{C} \backslash \overline{R_{b}(0 / 1)} \cup \overline{R_{b}(1 / 2)}$. Now, assume that there exists an angle $\theta \in \Theta$ such that the dynamical ray $R_{b}(\theta)$ bifurcates. Then, it bifurcates on a preimage of the escaping critical point $\omega_{2}(b)$ and one of its forward image bifurcates on $\omega_{2}(b)$. 
But this contradicts the fact that the forward orbit of the ray $R_{b}(\theta)$ is contained in $\mathbb{C} \backslash V_{2}(b)$ which does not contain $\omega_{2}(b)$.

Next, observe that the mapping $h: \mathcal{W}_{0} \times X_{b_{1}} \rightarrow X_{b}$ defined by $h(b, z)=$ $\varphi_{b}^{-1} \circ \varphi_{b_{1}}(z)$ is a holomorphic motion of $X_{b_{1}}$ parametrized by $b \in \mathcal{W}_{0}$. The $\lambda$ Lemma by Mañe, Sad and Sullivan [MSS] shows that $h$ extends to a holomorphic motion of the closure $\overline{X_{b_{1}}}$ of $X_{b_{1}}$ in $\mathbb{C}$. Since $\mathcal{W}_{0}$ is a simply connected Riemann surface, Slodkowski's Theorem (see [S1] [D2]) shows that one can in fact extend $h$ to a holomorphic motion of the whole complex plane $\mathbb{C}$, still parametrized by $b \in \mathcal{W}_{0}$. We will keep the notation $h$ for this extension. The mapping $z \mapsto$ $h_{b}(z)=h(b, z)$ is a $K(b)$-quasi-conformal homeomorphism, where $K(b)$ is the exponential of the hyperbolic distance between $b_{1}$ and $b$ in $\mathcal{W}_{0}$. It maps the set of dynamical rays $X_{b_{1}}$ to the set of dynamical rays $X_{b}$, and $h_{b}\left(\overline{X_{b_{1}}} \backslash X_{b_{1}}\right)=$ $\overline{X_{b}} \backslash X_{b}$. Since $\Theta$ is closed, the set $J_{b}$ is contained in the Julia set $J\left(f_{b}\right)$. Since $K\left(f_{b}\right)$ is full, the set $K_{b}$ is contained in the filled-in Julia set $K\left(f_{b}\right)$ Finally, $h_{b}$ provides a quasi-conformal homeomorphism between $K_{b}$ and $K_{b_{1}}$, and since $K_{b_{1}}$ is quasi-conformally homeomorphic to the quadratic Julia set $K\left(\lambda z+z^{2}\right)$ (see propositions 5 and 7 ), Theorem $\mathrm{A}$ is proved.

Observe that the mapping $h_{b}$ conjugates the polynomials $f_{b_{1}}$ and $f_{b}$ on the set of rays $X_{b_{1}}$, i.e., for any $z \in X_{b_{1}}$ we have $h_{b} \circ f_{b_{1}}=f_{b} \circ h_{b}$. By continuity of $h_{b}$, this property holds on the closure $\overline{X_{b_{1}}}$ and in particular on $J_{b_{1}}$.

Observe also that the fixed point 0 never belong to the set $X_{b}$ so that the set $X_{b} \cup\{0\}$ moves holomorphically when $b$ moves in $\mathcal{W}_{0}$. In particular, we can choose the extension $h$ so that $h(b, 0)=0$ for any $b \in \mathcal{W}_{0}$. Since $0 \in K_{b_{1}}$, this shows that for any $b \in \mathcal{W}_{0}, 0$ belongs to $K_{b}$.

We finally would like to mention that we could choose the extension of $h$ so that $h_{b}$ conjugates the polynomials $f_{b_{1}}$ and $f_{b}$ on the whole set $K_{b_{1}}$, and such that the distributional derivative $\partial h_{b} / \partial \bar{z}$ vanishes on $K_{b_{1}}$. But this would require extra work and we will just mention the idea of the proof. We could first prove that for any $b \in \mathcal{W}_{0}$, there is a restriction of $f_{b}: U_{b}^{\prime} \rightarrow U_{b}$ to a neighborhood of $K_{b}$ which is a quadratic-like map. We could then prove as in proposition 5 that the hybrid class of this quadratic-like restriction contains the quadratic polynomial $z \mapsto \lambda z+z^{2}$. In particular, for any $b \in \mathcal{W}_{0}$, the polynomial-like maps $f_{b}: U_{b}^{\prime} \rightarrow U_{b}$ and $f_{b_{1}}: U_{b_{1}}^{\prime} \rightarrow U_{b_{1}}$ would be hybrid conjugate, i.e., there would exist a quasi-conformal homeomorphism $h_{b}: U_{b_{1}} \rightarrow U_{b}$ such that $h_{b} \circ f_{b_{1}}=f_{b} \circ h_{b}$ on $U_{b_{1}}^{\prime}$ and such that the distributional derivative $\partial h_{b} / \partial \bar{z}$ vanishes on $K_{b_{1}}$. We would finally have to prove that the restriction of the mapping $(b, z) \mapsto h_{b}(z)$ to $\mathcal{W}_{0} \times K_{b_{1}}$ gives a holomorphic motion $K_{b_{1}}$ extending $h$.

\section{The dyadic wakes $\mathcal{W}_{\vartheta}$.}

Observe that in the wake $\mathcal{W}_{0}$ we see a copy $M^{\prime}$ of a Mandelbrot set, with root point at $b_{0}$. In this section, we will explain why we see such a copy, and we will determine a Cantor set $\Theta^{\prime}$ such that the boundary of $M^{\prime}$ is the accumulation set of the parameter rays $\mathcal{R}_{\lambda}(\theta), \theta \in \Theta^{\prime}$.

The reason why such a copy appears is that for any $b \in \mathcal{W}_{0}$, the mapping $f_{b}: U_{2} \rightarrow V_{2}$ is a ramified covering of degree 2, ramified at $\omega_{2}$. The sets $U_{2}$ and $V_{2}$ are topological disks and $U_{2} \subset V_{2}$, and the family $\left(f_{b}: U_{2} \rightarrow V_{2}\right)_{b \in \mathcal{W}_{0}}$ is almost a Mandelbrot-like family (see [DH2]). The problem is that $U_{2}$ is not 
relatively compact in $V_{2}$. If $\lambda \neq 1$, one can cut along equipotentials and thicken domains (see $[\mathrm{M}]$ ) to construct quadratic-like mappings. Such an approach has already been developed by Epstein and Yampolsky [EY] who proved that there exists a homeomorphism $\chi: M^{\prime} \backslash\left\{b_{0}\right\} \rightarrow M \backslash\{1 / 4\}$ such that for any $b \in M^{\prime}$, there exists a quadratic-like restriction $f_{b}: V_{b}^{\prime} \rightarrow V_{b}$ which is hybrid conjugate to $z \mapsto z^{2}+\chi(b)$.

The case $\lambda=1$ is different and less understood. Indeed, when $\lambda=1$, the fixed point $\beta(b)$ is parabolic with multiplier 1 . In this case, no more thickening is possible. We would like to mention that in [Ha], Haïssinsky has made a major step in the direction of proving that in the case $\lambda=1$, the set $M^{\prime}$ is nevertheless homeomorphic to the Mandelbrot set. Since the thickening is not possible when $\lambda=1$, we need to adopt an approach that is not based on surgery.

\section{Definition 13.}

$$
\begin{gathered}
K_{b}^{\prime}=\left\{z \in K\left(f_{b}\right) \mid(\forall n \geq 0) f_{b}^{\circ n}(z) \in \overline{U_{2}}\right\}, \quad J_{b}^{\prime}=\partial K_{b}^{\prime} \quad \text { and } \\
M^{\prime}=\left\{b_{0}\right\} \cup\left\{b \in \mathcal{W}_{0} \mid K_{b}^{\prime} \text { is connected }\right\} .
\end{gathered}
$$

Proposition 10. The sets $K_{b}^{\prime}$ and $M^{\prime}$ have the following properties:

1. for any $b \in \mathcal{W}_{0}, K_{b}^{\prime}$ is a compact set, $K_{b}^{\prime} \subset K\left(f_{b}\right)$ and $J_{b}^{\prime} \subset J\left(f_{b}\right)$;

2. a parameter $b \in \mathcal{W}_{0}$ belongs to $M^{\prime}$ if and only if $\omega_{2}(b)$ belongs to $K_{b}^{\prime}$;

3. $M^{\prime}$ is a compact subset of $M_{\lambda}$ and $\partial M^{\prime} \subset \partial M_{\lambda}$.

4. if $b \in \mathcal{W}_{0} \backslash M^{\prime}$, then any cycle of $f_{b}$ which entirely lies in $U_{2}$ is repelling.

Proof.

1. For any $b$ in $\mathcal{W}_{0}$, we have

$$
K_{b}^{\prime}=\bigcap_{n \geq 0} K_{n}, \quad \text { where } \quad K_{0}=K\left(f_{b}\right) \cap \overline{U_{2}} \quad \text { and } \quad K_{n+1}=\left(\left.f_{b}\right|_{\overline{U_{2}}}\right)^{-1}\left(K_{n}\right) .
$$

Each $K_{n}$ is compact. Hence, $K_{b}^{\prime}$ is also compact. By definition, $K_{b}^{\prime} \subset K\left(f_{b}\right)$. Given any point $z$ in a connected component $U$ of the interior of $K\left(f_{b}\right)$, if $f_{b}^{\circ n}(z) \notin \overline{U_{2}}$, for some integer $n \geq 0$, then $f_{b}^{\circ n}(U)$ entirely lies in $\mathbb{C} \backslash \overline{U_{2}}$. Hence, $\partial K_{b}^{\prime} \subset \partial K\left(f_{b}\right)$, i.e., $J_{b}^{\prime} \subset J\left(f_{b}\right)$.

2. Let us now consider a parameter $b \in \mathcal{W}_{0}$. If $\omega_{2}(b) \in K_{b}^{\prime}$, then $\omega_{2}(b) \in K\left(f_{b}\right)$, and $K_{0}=K\left(f_{b}\right) \cap \overline{U_{2}}$ is connected. By induction, assume $K_{n}$ is connected. Then, since $\omega_{2}(b) \in K_{b}^{\prime}$, we see that $f_{b}\left(\omega_{2}(b)\right) \in K_{n}$, and $K_{n+1}$ is also connected. Hence, $K_{b}^{\prime}$ is the intersection of a nested sequence of connected closed sets. Thus, $K_{b}^{\prime}$ is connected and $b \in M^{\prime}$. Conversely, if $\omega_{2}(b) \notin K_{b}^{\prime}$, there exists an integer $n \geq 1$ such that $f_{b}^{\circ n}\left(\omega_{2}(b)\right) \notin \overline{U_{2}}$. Since $K_{0} \in \overline{U_{2}}$, we see that $K_{n}$ has at least two connected components. This shows that $K_{b}^{\prime}$ is not connected and $b \notin M^{\prime}$.

3. If $b$ belongs to $M^{\prime}$, then $\omega_{2}(b) \in K_{b}^{\prime} \subset K\left(f_{b}\right)$. Hence, $M^{\prime} \subset M_{\lambda}$. We have seen that $b \in \mathcal{W}_{0} \backslash M^{\prime}$ if and only if there exists an integer $n \geq 1$ such that $f_{b}^{\circ n}\left(\omega_{2}(b) \notin \overline{U_{2}}\right.$. Since $\overline{U_{2}}$ moves holomorphically, hence continuously, when $b$ moves in $\mathcal{W}_{0}$, we see that this is an open condition. Hence, $\mathcal{W}_{0} \backslash M^{\prime}$ is open in $\mathcal{W}_{0}$. Since the closure of $M^{\prime}$ is contained in the closure of $M_{\lambda}$, since $M_{\lambda} \cap \partial \mathcal{W}_{0}=\left\{b_{0}\right\}$, and since by definition $M^{\prime} \cap \partial \mathcal{W}_{0}=\left\{b_{0}\right\}$, we see that $M^{\prime}$ is closed, hence 
compact. Let us now show that $\partial M^{\prime} \subset \partial M_{\lambda}$. Take a parameter $b \neq b_{0}$ in the boundary of $M^{\prime}$. Then in any neighborhood $\mathcal{U} \subset \mathcal{W}_{0}$ of $b$, we can find a parameter $b^{\prime} \in \mathcal{U} \backslash M^{\prime}$ so that there exists an integer $n \geq 1$ with $f_{b^{\prime}}^{\circ n}\left(\omega_{2}\left(b^{\prime}\right)\right) \notin \overline{U_{2}}$. Since $f_{b}^{\circ n}\left(\omega_{2}(b)\right) \in \overline{U_{2}}$, and since the boundary of $U_{2}$ moves holomorphically when the parameter moves in $\mathcal{U}$, we can find a parameter $b^{\prime \prime} \in \mathcal{U}$ such that $f_{b^{\prime \prime}}^{\circ n}\left(\omega_{2}\left(b^{\prime \prime}\right)\right) \in \partial U_{2}$. There are two possibilities:

either $f_{b^{\prime \prime}}^{\text {on }}\left(\omega_{2}\left(b^{\prime \prime}\right)\right)$ belongs to a dynamical ray; in that case $b^{\prime \prime} \notin M_{\lambda}$;

or $f_{b^{\prime \prime}}^{\circ n}\left(\omega_{2}\left(b^{\prime \prime}\right)\right)$ is one of the two points $\beta\left(b^{\prime \prime}\right)$ or $\beta_{1}\left(b^{\prime \prime}\right)$; in that case the critical point $\omega_{2}(b)$ is eventually mapped to a repelling fixed point, and it is well-known that $b^{\prime \prime} \in \partial M_{\lambda}$.

4. Assume that $b \in \mathcal{W}_{0} \backslash M^{\prime}$. Then there exists a smallest integer $n \geq 1$ such that $f_{b}^{o n}\left(\omega_{2}(b)\right) \notin U_{2}$. Define $U^{\prime \prime}$ to be the $n$-th preimage of $U_{2}$ by $\left.f_{b}\right|_{U_{2}}$ and define $U^{\prime}$ to be the image of $U^{\prime \prime}$ by $f_{b}$. The, $f_{b}: U^{\prime \prime} \rightarrow U^{\prime}$ is a non-ramified covering map of degree 2. Hence, there are two well-defined inverse branches $g_{1}: U^{\prime} \rightarrow U^{\prime \prime}$ and $g_{2}: U^{\prime} \rightarrow U^{\prime \prime}$. By Schwarz's lemma, those two branches are contracting for the Poincaré metric of $U^{\prime \prime}$ and thus, every periodic orbit of $f_{b}$ contained in $U^{\prime}$ is repelling (there may be periodic orbit contained in the closure of $U^{\prime}$, but we are only concerned by the ones contained inside $U^{\prime}$ ).

Definition 14. We define $\Theta^{\prime} \subset \mathbb{R} / \mathbb{Z}$ to be the set of angles $\theta$ such that for any $n \geq 0,3^{n} \theta \in[1 / 2,1] \bmod 1$. We also define $\mathcal{X}^{\prime}$ to be the set of parameter rays

$$
\mathcal{X}^{\prime}=\bigcup_{\theta \in \Theta^{\prime}} \mathcal{R}_{\lambda}(\theta / 3),
$$

and for any $b \in M^{\prime}$, we define $X_{b}^{\prime}$ to be the set of dynamical rays

$$
X_{b}^{\prime}=\bigcup_{\theta \in \Theta^{\prime}} R_{b}(\theta)
$$

Remark. The set $\Theta^{\prime}$ is the set of angles $\theta^{\prime}$ that can be written in base 3 with only 1's and 2's. It is a Cantor set, invariant under multiplication by 3 . In fact, $\theta \in \Theta^{\prime}$ if and only if $\theta-1 / 2 \in \Theta$. Observe also that for any $\theta \in \Theta^{\prime}$, the two angles $\theta / 3+1 / 3$ and $\theta / 3+2 / 3$ also belong to $\Theta^{\prime}$.

Definition 15. We will say that $b \in M^{\prime}$ is a tip of $M^{\prime}$ if and only if the orbit of $\omega_{2}(b)$ is eventually mapped to $\beta(b)$, i.e., if there exists an integer $k \geq 1$ such that $f^{\circ k}\left(\omega_{2}(b)\right)=\beta(b)$.

Proposition 11. We have the following dynamical result:

1. for any parameter $b \in M^{\prime}$, we have $J_{b}^{\prime}=\overline{X_{b}^{\prime}} \backslash X_{b}^{\prime}$, where the closure is taken in $\mathbb{C}$;

2. for any $b \in M^{\prime}$, any $z \in J_{b}^{\prime}$ which is eventually mapped to $\beta(b)$ is the landing point of at least two rays $R_{b}\left(\theta^{-}\right)$and $R_{b}\left(\theta^{+}\right)$, where $\theta^{ \pm} \in \Theta^{\prime}$. Moreover, if $f^{\circ k}(z)=\beta$ and $\left(f^{\circ k}\right)^{\prime}(z) \neq 0$, then, there are exactly two dynamical rays landing at $z$.

The parameter counterpart of this statement is the following:

3. the boundary of $M^{\prime}$ is the accumulation set of $\mathcal{X}^{\prime}: \partial M^{\prime}=\overline{\mathcal{X}^{\prime}} \backslash \mathcal{X}^{\prime}$; 
4. for any tip $b \in M^{\prime}$, there are exactly two angles $\theta^{-} \in \Theta^{\prime}$ and $\theta^{+} \in \Theta^{\prime}$ such that $\omega_{2}^{\prime}(b)$ is the landing point of the two dynamical rays $R_{b}\left(\theta^{-} / 3\right)$ and $R_{b}\left(\theta^{+} / 3\right)$. Furthermore, the parameter rays $\mathcal{R}_{\lambda}\left(\theta^{-} / 3\right)$ and $\mathcal{R}_{\lambda}\left(\theta^{+} / 3\right)$ land at $b \in M^{\prime}$.

Figure 12 shows the set $\mathcal{X}^{\prime}$ of parameter rays and the set $M^{\prime}$.

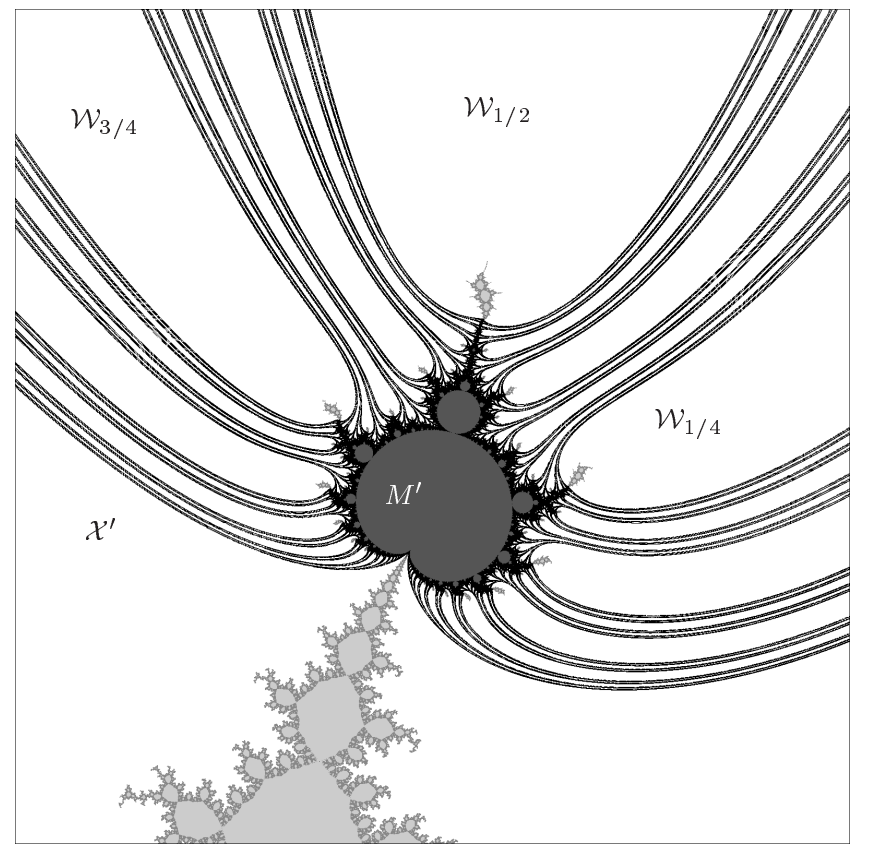

Fig. 12. The set $\mathcal{X}^{\prime}$ of parameter rays and the set $M^{\prime}$.

Proof.

1. Let us fix a parameter $b \in M^{\prime}$. Then, the dynamical rays $R_{b}(\theta), \theta \in \Theta^{\prime}$, do not bifurcate and the set $X_{b}^{\prime}$ is exactly the set of rays in $\overline{U_{2}(b)}$ whose forward orbit remains in $\overline{U_{2}(b)}$. Take any point $z_{0}$ in the accumulation set of $X_{b}^{\prime}$. Since $\Theta^{\prime}$ is closed, $z_{0} \in J\left(f_{b}\right)$. Then, since $\Theta^{\prime}$ is forward invariant by multiplication by 3 , for any integer $n \geq 0$, the point $z_{n}=f_{b}^{\circ n}\left(z_{0}\right)$ is in the accumulation set of $X_{b}^{\prime}$. Since $X_{b}^{\prime} \subset \overline{U_{2}(b)}$, we obtain $z_{n} \in \overline{U_{2}(b)}$. But this precisely shows that $z_{0} \in J_{b}^{\prime}$. Hence $\overline{X_{b}^{\prime}} \backslash X_{b}^{\prime} \subset J_{b}^{\prime}$.

Conversely, given any point $z_{0} \in J_{b}^{\prime}$ and any connected neighborhood $W_{0}$ of $z_{0}$, we must show that $W_{0}$ contains points of $X_{b}^{\prime}$. Since $z_{0} \in J_{b}^{\prime}$, for any integer $n \geq 0$, the point $z_{n}=f_{b}^{\circ n}\left(z_{0}\right)$ belongs to $\bar{U}_{2}(b)$. Since $J_{b}^{\prime} \subset J\left(f_{b}\right)$ (see proposition 10), the family of iterates $f_{b}^{\circ n}: W_{0} \rightarrow \mathbb{C}$ is not normal. Hence, there exists a first integer $n \geq 0$ such that $W_{n}=f_{b}^{\circ n}\left(W_{0}\right)$ intersects $\mathbb{C} \backslash U_{2}$. Since $W_{n}$ is connected and contains the point $z_{n} \in \overline{U_{2}(b)}$, we see that $W_{n}$ intersects at least one of the rays $R_{b}(0 / 1), R_{b}(1 / 2), R_{b}(2 / 3)$, or $R_{b}(5 / 6)$. Besides, for any 
integer $k \in[0, n-1], W_{k}$ is contained in $U_{2}(b)$. Hence, $W_{0}$ intersect a ray which is eventually mapped to one of the rays $R_{b}(0 / 1), R_{b}(1 / 2), R_{b}(2 / 3)$, or $R_{b}(5 / 6)$ and whose forward orbit remains in $\overline{U_{2}}$. Such a ray necessarily belongs to the set $X_{b}^{\prime}$.

2. We only need to observe that for any $z \in J_{b}^{\prime}$, if there exists an integer $k \geq 0$ such that $f_{b}^{\circ k}(z)=\beta(b)$, then there exists a neighborhood $U$ of $z$ such that $f_{b}^{\circ k}: U \rightarrow f_{b}^{\circ k}(U)$ is a covering. This covering may be ramified if $z$ is a preimage of $\omega_{2}(b)$. However, by restricting $U$ if necessary, we may assume that $z$ is the only ramification point. Since the two rays $R_{b}(0 / 1)$ and $R_{b}(1 / 2)$ land at $\beta(b)$, there are at least two rays $R_{b}\left(\theta^{-}\right)$and $R_{b}\left(\theta^{+}\right)$that land at $z$, satisfying $f_{b}^{\circ k}\left(R_{b}\left(\theta^{-}\right)\right)=$ $R_{b}(0 / 1)$ and $f_{b}^{\circ k}\left(R_{b}\left(\theta^{+}\right)\right)=R_{b}(1 / 2)$. Finally, since the forward orbit of $z$ remains in $\overline{V_{2}}$, we immediately see that the forward orbit of $R_{b}\left(\theta^{ \pm}\right)$also remains in $\overline{V_{2}}$. Thus, $\theta^{ \pm} \in \Theta^{\prime}$. Furthermore, if $\left(f_{b}^{\circ k}\right)^{\prime}(z) \neq 0$, we have to show that there are exactly two dynamical rays landing at $z$. Since $R_{b}(0 / 1)$ is landing at $\beta(b)$, every dynamical ray landing at $\beta$ must have combinatorial rotation number $0 / 1$. Hence, the dynamical rays landing at $\beta(b)$ are exactly the rays $R_{b}(0 / 1)$ and $R_{b}(1 / 2)$. Since $f_{b}^{\circ k}$ is a local isomorphism at $z$, mapping $z$ to $\beta(b)$, there are exactly two dynamical rays landing at $z$.

3. Since $\Theta^{\prime}$ is closed, the accumulation set $\overline{\mathcal{X}^{\prime}} \backslash \mathcal{X}^{\prime}$ is contained in the boundary of $M_{\lambda}$. Given any parameter $b^{\prime}$ in this accumulation set, we want to show that $b^{\prime} \in M^{\prime}$. Since, by definition of $M^{\prime}$, the parameter $b_{0}$ belongs to $M^{\prime}$, we may assume that $b^{\prime} \neq b_{0}$. In this case, $b^{\prime} \in \mathcal{W}_{0}$. Given any parameter $b \in \mathcal{X}^{\prime}$, and any integer $n \geq 1$, the point $f_{b}^{\circ n}\left(\omega_{2}(b)\right)$ belongs to a dynamical ray $R_{b}\left(3^{n} \theta\right)$, for some $\theta \in \Theta^{\prime}$. Hence, the whole orbit $\left\{f_{b}^{\circ n}\left(\omega_{2}(b)\right)\right\}_{n \geq 0}$ belongs to $\overline{U_{2}(b)}$. Then, by continuity of $\overline{U_{2}(b)}$ at $b^{\prime} \in \mathcal{W}_{0}$, the whole orbit $\left\{f_{b^{\prime}}^{\circ n}\left(\omega_{2}\left(b^{\prime}\right)\right)\right\}_{n \geq 0}$ belongs to $\overline{U_{2}\left(b^{\prime}\right)}$. But since $b^{\prime} \in M_{\lambda}$, we know that $\omega_{2}\left(b^{\prime}\right) \in K\left(f_{b^{\prime}}\right)$. This shows that $b^{\prime} \in M^{\prime}$. Hence $\overline{\mathcal{X}^{\prime}} \backslash \mathcal{X}^{\prime} \subset \partial M^{\prime}$.

Conversely, we want to prove that $\partial M^{\prime} \subset \overline{\mathcal{X}^{\prime}} \backslash \mathcal{X}^{\prime}$. We know that $b_{0}$ is the landing point of the rays $\mathcal{R}_{\lambda}(1 / 6)$ and $\mathcal{R}_{\lambda}(1 / 3)$. Hence $b_{0} \in \overline{\mathcal{X}^{\prime}} \backslash \mathcal{X}^{\prime}$. Given any parameter $b_{*} \in \partial M^{\prime} \backslash\left\{b_{0}\right\} \subset \partial M_{\lambda} \cap \mathcal{W}_{0}$, and any neighborhood $\mathcal{U} \subset \mathcal{W}_{0}$ of $b_{*}$, we want to show that there exists a parameter $b \in \mathcal{U}$ such that one of the rays $R_{b}(\theta), \theta \in \Theta^{\prime}$ bifurcates on $\omega_{2}(b)$. Assume this is not the case. Then, the set $X_{b}^{\prime}=\bigcup_{\theta \in \Theta^{\prime}} R_{b}(\theta)$ moves holomorphically when $b \in \mathcal{U}$, and therefore $\overline{X_{b}^{\prime}}$ remains connected for all $b \in \mathcal{U}$ and $\overline{X_{b}^{\prime}} \backslash X_{b}^{\prime} \subset J\left(f_{b}\right)$. By proposition 10 we have $\partial M^{\prime} \subset \partial M_{\lambda}$, so there exists a parameter $b^{\prime} \in \mathcal{U}$ such that $\omega_{2}\left(b^{\prime}\right) \notin K\left(f_{b^{\prime}}\right)$. Since the rays $R_{b^{\prime}}(\theta), \theta \in \Theta^{\prime}$ do not bifurcate on $\omega_{2}\left(b^{\prime}\right)$ and since $\overline{X_{b^{\prime}}^{\prime}} \backslash X_{b^{\prime}}^{\prime} \subset J\left(f_{b}\right)$, we see that $\omega_{2}\left(b^{\prime}\right)$ does not belong to $\overline{X_{b^{\prime}}^{\prime}}$. Besides, since $b^{\prime}$ is in the wake $\mathcal{W}_{0}$, the critical point $\omega_{2}\left(b^{\prime}\right)$ is in the region $U_{2}\left(b^{\prime}\right)$ Hence, there exists an angle $\left.\theta_{1} \in\right] 1 / 2,2 / 3\left[\right.$ such that the dynamical rays $R_{b^{\prime}}\left(\theta_{1}\right)$ and $R_{b^{\prime}}\left(\theta_{1}+1 / 3\right)$, bifurcate on $\omega_{2}\left(b^{\prime}\right)$. Since the set $R_{b^{\prime}}\left(\theta_{1}\right) \cup R_{b^{\prime}}\left(\theta_{1}+1 / 3\right) \cup\left\{\omega_{2}\left(b^{\prime}\right)\right\}$ does not intersect and does not disconnect $\overline{X_{b}^{\prime}}$, and since it separates $\beta\left(b^{\prime}\right) \in X_{b^{\prime}}^{\prime}$ and $\beta_{2}\left(b^{\prime}\right) \in X_{b^{\prime}}^{\prime}$, we get a contradiction.

4. Let us now consider a tip $b_{*} \in M^{\prime}$. Then, $f_{b_{*}}\left(\omega_{2}\left(b_{*}\right)\right) \in J_{b_{*}}^{\prime}$, there exists a smallest integer $k \geq 1$ such that $f_{b_{*}}^{\circ k}\left(\omega_{2}\left(b_{*}\right)\right)=\beta_{2}\left(b_{*}\right)$ and $f_{b_{*}}^{\circ(k-1)}$ is a local 
isomorphism at $f_{b_{*}}\left(\omega_{2}\left(b_{*}\right)\right)$. Hence, the dynamical statement shows that there are exactly two dynamical rays landing at $f_{b_{*}}\left(\omega_{2}\left(b_{*}\right)\right)$. Those rays are of the form $R_{b_{*}}\left(\theta^{+}\right)$and $R_{b_{*}}\left(\theta^{-}\right), \theta^{ \pm} \in \Theta^{\prime}$.

We will now show that the parameter ray $\mathcal{R}_{\lambda}\left(\theta^{+} / 3\right)$ land at the parameter $b_{*}$. A similar proof can be carried out for the parameter ray $\mathcal{R}_{\lambda}\left(\theta^{-} / 3\right)$. Observe that the two dynamical rays $R_{b_{*}}\left(\theta^{-} / 3\right)$ and $R_{b_{*}}\left(\theta^{+} / 3\right)$ land at $\omega_{2}^{\prime}\left(b_{*}\right)$. Besides, since the $k-1$ first iterates of $\omega_{2}\left(b_{*}\right)$ omit the rays $R_{b_{*}}(0 / 1)$ and $R_{b_{*}}(1 / 2)$, and since the rays $R_{b}(0 / 1)$ and $R_{b}(1 / 2)$ move holomorphically when $b \in \mathcal{W}_{0}$, it follows that there exist a neighborhood $\mathcal{U} \subset \mathcal{W}_{0}$ of $b_{*}$ such that for any $b \in \mathcal{U}$ and any $i \leq k-1, f_{b}^{\circ i}\left(\omega_{2}(b)\right)$ omits the two rays $R_{b}(0 / 1)$ and $R_{b}(1 / 2)$. In particular, for any $b \in \mathcal{U}$, the two dynamical rays $R_{b}\left(\theta^{-}\right)$and $R_{b}\left(\theta^{+}\right)$do not bifurcate. Pullingback once more, we see that for any $b \in \mathcal{U}$, the dynamical rays $R_{b}\left(\theta^{-} / 3\right)$ and $R_{b}\left(\theta^{+} / 3\right)$ do not bifurcate when $b \in \mathcal{U}$, and so, move holomorphically when $b$ moves in $\mathcal{U}$. Next, for every $\eta \in\left[0,+\infty\left[\right.\right.$, define $h_{\eta}: \mathcal{U} \rightarrow \mathbb{C}$ to be the holomorphic function

$$
h_{\eta}(b)=\varphi_{b}^{-1}\left(e^{\eta+2 i \pi \theta^{+} / 3}\right) .
$$

When $\eta$ tends to 0 , one can show that $h_{\eta}$ converges uniformly on $\mathcal{U}$ to a function $h_{0}$ (this is in fact the way one proves that the holomorphic motion of the ray extends to its closure). For any $b \in \mathcal{U}, h_{0}(b)$ is the landing point of the dynamical ray $R_{b}\left(\theta^{+}\right)$. Moreover, the function $h_{0}-\omega_{2}^{\prime}$ vanishes at $b_{*}$. Besides, it does not vanish on $\mathcal{U} \backslash M_{\lambda}$ since for any $b \in \mathcal{U} \backslash M_{\lambda}, \omega_{2}^{\prime}(b) \notin K\left(f_{b}\right)$, whereas $h_{0}(b) \in K\left(f_{b}\right)$. Let us assume that the parameter ray $\mathcal{R}_{\lambda}\left(\theta^{+} / 3\right)$ does not land at $b_{*}$. Then there exist a neighborhood $\mathcal{U}$ and a sequence $\eta_{k} \searrow 0$ such that $\Phi_{\lambda}^{-1}\left(e^{\eta_{k}+2 i \pi \theta^{+} / 3}\right) \notin \mathcal{U}$, i.e., the function $h_{\eta_{k}}-\omega_{2}^{\prime}$ does not vanish on $\mathcal{U}$. Then, Hurwitz's theorem shows that $h_{0}-\omega_{2}^{\prime}$ either does not vanish on $\mathcal{U}$, or vanishes everywhere on $\mathcal{U}$. This is in contradiction to the previous observation. Hence, the parameter ray $\mathcal{R}_{\lambda}\left(\theta^{+} / 3\right)$ lands at $b_{*}$.

Remark. We don't claim that the only rays accumulating on $J_{b}^{\prime}$ are rays of the form $R_{b}(\theta), \theta \in \Theta^{\prime}$, or that the only rays accumulating on $M^{\prime}$ are rays of the form $\mathcal{R}_{\lambda}(\theta / 3), \theta \in \Theta^{\prime}$. This would be of the same order of difficulty as proving that for a quadratic polynomial, the only dynamical ray accumulating the $\beta$ fixed point is the ray of angle $0 / 1$. In the case of Cremer polynomials, this is not known.

We will now consider the unbounded connected components of

$$
\mathcal{W}_{0} \backslash \bigcup_{\theta \in \Theta^{\prime}} \overline{\mathcal{R}_{\lambda}(\theta / 3)}
$$

We will show that those connected components are naturally indexed by the dyadic angles $\vartheta=(2 p+1) / 2^{k}, k \geq 1$ and $2 p+1<2^{k}$, and we will denote them by $\mathcal{W}_{\vartheta}$. We will also show that the boundary of a component $\mathcal{W}_{\vartheta}$ is the union of two parameter rays $\mathcal{R}_{\lambda}\left(\vartheta^{-} / 3\right)$ and $\mathcal{R}_{\lambda}\left(\vartheta^{+} / 3\right), \vartheta^{ \pm} \in \Theta^{\prime}$, that land at a common parameter $b_{\vartheta} \in M^{\prime}$.

In the next section, we will show that for every dyadic angle $\vartheta, M_{\lambda} \cap \overline{\mathcal{W}_{\vartheta}}$ contains a quasi-conformal copy $\mathcal{K}_{\vartheta}$ of the filled-in Julia set $K\left(\lambda z+z^{2}\right)$, such that $b_{\vartheta} \in \partial \mathcal{K}_{\vartheta} \subset \partial M_{\lambda}$. 
Definition 16. Any dyadic angle $\vartheta=(2 p+1) / 2^{k}, k \geq 1$ and $0<2 p+1<2^{k}$, can be expressed in a unique way as a finite sum

$$
\frac{2 p+1}{2^{k}}=\sum_{i=1}^{k} \frac{\varepsilon_{i}}{2^{i}},
$$

where each $\varepsilon_{i}, i=1, \ldots k$, takes the value 0 or 1 . We define $\vartheta^{-}$and $\vartheta^{+}$by the formulae:

$$
\vartheta^{-}=\sum_{i=1}^{k} \frac{\varepsilon_{i}+1}{3^{i}}, \quad \text { and } \quad \vartheta^{+}=\vartheta^{-}+\frac{1}{2 \cdot 3^{k}} .
$$

Remark. There are two ways of writing a dyadic number $\vartheta$ in base 2 :

$$
\vartheta=0 . \varepsilon_{1} \varepsilon_{2} \ldots \varepsilon_{k-1} 01111 \ldots=0 . \varepsilon_{1} \varepsilon_{2} \ldots \varepsilon_{k-1} 10000 \ldots
$$

Read those two numbers in base 3 and add $1 / 2$. You will obtain $\vartheta^{-}$and $\vartheta^{+}$.

Proposition 12. Given any dyadic angle $\vartheta=(2 p+1) / 2^{k}, k \geq 1,0<2 p+1<$ $2^{k}$, the two parameter rays $\mathcal{R}_{\lambda}\left(\vartheta^{-} / 3\right)$ and $\mathcal{R}_{\lambda}\left(\vartheta^{+} / 3\right)$ land at a common tip $b_{\vartheta} \in M^{\prime}$. More precisely,

$$
f_{b_{\vartheta}}^{o(k+1)}\left(\omega_{2}\left(b_{\vartheta}\right)\right)=\beta\left(b_{\vartheta}\right),
$$

and the two dynamical rays $R_{b_{\vartheta}}\left(\vartheta^{-} / 3\right)$ and $R_{b_{\vartheta}}\left(\vartheta^{+} / 3\right)$ land at $\omega_{2}^{\prime}\left(b_{\vartheta}\right)$.

Proof.

Step 1. Let us first prove that the parameter ray $\mathcal{R}_{\lambda}\left(\vartheta^{-} / 3\right)$ land either at $b_{0}$ or at a tip $b_{\vartheta} \in M^{\prime}$ (a similar proof works for the parameter ray $\mathcal{R}_{\lambda}\left(\vartheta^{+} / 3\right)$ ). The argument we use is very similar to the one written in the Orsay notes [DH1]. Let us choose any parameter $b_{\vartheta}$ in the accumulation set of the parameter ray $\mathcal{R}_{\lambda}\left(\vartheta^{-} / 3\right)$ and assume $b_{\vartheta} \neq b_{0}$. Then, $b_{\vartheta}$ belongs to the wake $\mathcal{W}_{0}$ and proposition 11 shows that $b_{\vartheta} \in M^{\prime}$. Moreover, observe that $3^{k} \vartheta^{-} \equiv 0 \bmod 1$. Hence, if $b$ is the point of the parameter ray $\mathcal{R}_{\lambda}\left(\vartheta^{-} / 3\right)$ of potential $\eta$, then $f_{b}^{\circ(k+1)}\left(\omega_{2}(b)\right)$ is the point of the dynamical ray $R_{b}(0 / 1)$ of potential $3^{k+1} \eta$. Since $b_{\vartheta}$ is in the wake $\mathcal{W}_{0}$, the dynamical ray $R_{b}(0 / 1)$ moves holomorphically in a neighborhood of $b_{\vartheta}$ and lands at $\beta(b)$. Hence, by continuity as $\eta$ tends to 0 , we obtain that $f_{b_{\vartheta}}^{\circ(k+1)}\left(\omega_{2}\left(b_{\vartheta}\right)\right)=\beta\left(b_{\vartheta}\right)$. This shows that $b_{\vartheta}$ is a tip of $M^{\prime}$. Furthermore, the set of parameters $b$ such that $f_{b}^{\circ(k+1)}\left(\omega_{2}(b)\right)=\beta(b)$ is discrete and the accumulation set of the parameter ray $\mathcal{R}_{\lambda}\left(\vartheta^{-} / 3\right)$ is connected. Hence, the parameter ray $\mathcal{R}_{\lambda}\left(\vartheta^{-} / 3\right)$ land either at $b_{0}$ or at a tip $b_{\vartheta} \in M^{\prime}$.

Let us now show that if the parameter ray $\mathcal{R}_{\lambda}\left(\vartheta^{-} / 3\right)$ lands at a tip $b_{\vartheta} \in M^{\prime}$, then the dynamical ray $R_{b_{\vartheta}}\left(\vartheta^{-} / 3\right)$ lands at $\omega_{2}^{\prime}\left(b_{\vartheta}\right)$. For this purpose we need the following lemma:

Lemma 12. Let $\theta$ be any angle such that $3^{k} \theta=0 \bmod 1$ or $3^{k} \theta=1 / 2 \bmod 1$ for some integer $k$, and let $b_{*}$ be any parameter in $M_{\lambda} \cap \mathcal{W}_{0}$. Then the dynamical ray $R_{b_{*}}(\theta)$ lands at a preimage $z_{*}$ of $\beta\left(b_{*}\right)$. Assume $z_{*}$ is not a preimage of the critical point $\omega_{2}\left(b_{*}\right)$. Then, when $b$ moves in a sufficiently small neighborhood of $b_{*}$, the ray $R_{b}(\theta)$ does not bifurcate, and thus, moves holomorphically. 
Proof.

We will treat the case $3^{k} \theta=1 / 2 \bmod 1$. The other case is similar. Since $b_{*} \in M_{\lambda}$, the dynamical ray $R_{b_{*}}(\theta)$ does not bifurcate. Besides, $3^{k} \theta=1 / 2 \bmod 1$, we have

$$
f_{b_{*}}^{\circ k}\left(R_{b_{*}}(\theta)\right)=R_{b_{*}}(1 / 2) .
$$

Since the ray $R_{b_{*}}(1 / 2)$ lands at $\beta\left(b_{*}\right)$, we see that the ray $R_{b_{*}}(\theta)$ lands at a preimage $z_{*}$ of $\beta\left(b_{*}\right)$. The lemma now follows directly from [DH1], Proposition 3 , exposé 8 .

We can apply the above lemma to the angle $\vartheta^{-} / 3$ and the parameter $b_{\vartheta}$. It shows that the ray $R_{b_{\vartheta}}\left(\vartheta^{-} / 3\right)$ lands at a preimage $z_{\vartheta}$ of $\beta\left(b_{\vartheta}\right)$.

If $z_{\vartheta}$ is not a preimage of the critical point $\omega_{2}\left(b_{\vartheta}\right)$ then the ray moves holomorphically in a neighborhood of $b_{\vartheta}$. We define $b_{\eta}$ to be the point of potential $\eta$ on the parameter ray $\mathcal{R}_{\lambda}\left(\vartheta^{-} / 3\right)$. Then $\omega_{2}^{\prime}\left(b_{\eta}\right)$ is the point of potential $\eta$ on the dynamical ray $R_{b_{\eta}}\left(\vartheta^{-} / 3\right)$. When $\eta$ tends to $0, b_{\eta}$ tends to $b_{\vartheta}$ and $\omega_{2}^{\prime}\left(b_{\eta}\right)$ converges to the landing point of the dynamical ray $R_{b_{\vartheta}}\left(\vartheta^{-} / 3\right)$. By continuity of the function $\omega_{2}^{\prime}$, it proves that the dynamical ray $R_{b_{\vartheta}}\left(\vartheta^{-} / 3\right)$ lands at $\omega_{2}^{\prime}\left(b_{\vartheta}\right)$.

Hence, the only remaining difficulty is proving that $z_{\vartheta}$ is not a preimage of the critical point $\omega_{2}\left(b_{\vartheta}\right)$. If this were the case, one could find an integer $k_{1}$ such that $f_{b_{\vartheta}}^{\circ k_{1}}\left(z_{\vartheta}\right)=\omega_{2}\left(b_{\vartheta}\right)$. Note that $k_{1} \geq 1$ since $\omega_{2}\left(b_{\vartheta}\right)$ and the dynamical ray $R_{b_{\vartheta}}\left(\vartheta^{-} / 3\right)$ are separated by $R_{b_{\vartheta}}(0 / 1) \cup R_{b_{\vartheta}}(1 / 2) \cup\left\{\beta\left(b_{\vartheta}\right)\right\}$. Since $\omega_{2}\left(b_{\vartheta}\right)$ is strictly preperiodic (this is our assumption that $b_{\vartheta} \neq b_{0}$ ), iterating once more, we know that $f_{b_{\vartheta}}^{\circ\left(k_{1}+1\right)}\left(z_{\vartheta}\right)=f_{b_{\vartheta}}\left(\omega_{2}\left(b_{\vartheta}\right)\right)$ is not a preimage of $\omega_{2}\left(b_{\vartheta}\right)$ and is the landing point of the dynamical ray $R_{b_{\vartheta}}\left(3^{k_{1}} \vartheta^{-}\right)$. Hence, we can apply lemma 12. It shows that the ray $R_{b}\left(3^{k_{1}} \vartheta^{-}\right)$moves holomorphically in a neighborhood of $b_{\vartheta}$. Then again, defining $b_{\eta}$ to be the point of potential $\eta$ on the parameter ray $\mathcal{R}_{\lambda}\left(\vartheta^{-} / 3\right)$, we get by continuity that $f_{b_{\vartheta}}^{\circ\left(k_{1}+1\right)}\left(\omega_{2}\left(b_{\vartheta}\right)\right)=f_{b_{\vartheta}}\left(\omega_{2}\left(b_{\vartheta}\right)\right)$. Hence, either $f_{b_{\vartheta}}^{\circ k_{1}}\left(\omega_{2}\left(b_{\vartheta}\right)\right)=\omega_{2}\left(b_{\vartheta}\right)$ or $f_{b_{\vartheta}}^{\circ k_{1}}\left(\omega_{2}\left(b_{\vartheta}\right)\right)=\omega_{2}^{\prime}\left(b_{\vartheta}\right)$. The first case is not possible since $\omega_{2}\left(b_{\vartheta}\right)$ is not periodic. The second case is also impossible since $b_{\vartheta} \in$ $M^{\prime}$ and thus $\omega_{2}\left(b_{\vartheta}\right)$ and $\omega_{2}^{\prime}\left(b_{\vartheta}\right)$ are separated by $R_{b_{\vartheta}}(0 / 1) \cup R_{b_{\vartheta}}(1 / 2) \cup\left\{\beta\left(b_{\vartheta}\right)\right\}$.

Step 2. Let us now show that the parameter rays $\mathcal{R}_{\lambda}\left(\vartheta^{+} / 3\right)$ and $\mathcal{R}_{\lambda}\left(\vartheta^{-} / 3\right)$ land at the same parameter. Either, both of them land at $b_{0}$, or one of them land at a tip $b_{\vartheta} \neq b_{0}$ of $M^{\prime}$. Without loss of generality, assume that $\mathcal{R}_{\lambda}\left(\vartheta^{-} / 3\right)$ lands at $b_{\vartheta} \neq b_{0}$. We just proved in step 1 that the dynamical ray $R_{b_{\vartheta}}\left(\vartheta^{-} / 3\right)$ lands at $\omega_{2}^{\prime}\left(b_{\vartheta}\right)$. Proposition $11(4)$ shows that there are exactly two rays landing at $\omega_{2}^{\prime}\left(b_{\vartheta}\right)$. It is not difficult to check that the other dynamical ray landing at $\omega_{2}^{\prime}\left(b_{\vartheta}\right)$ is $R_{b_{\vartheta}}\left(\vartheta^{+} / 3\right)$. Proposition 11 (4) then shows that the parameter ray $\mathcal{R}_{\lambda}\left(\vartheta^{+} / 3\right)$ lands at $b_{\vartheta}$.

Step 3. We now need to prove that the parameter rays $\mathcal{R}_{\lambda}\left(\vartheta^{+} / 3\right)$ and $\mathcal{R}_{\lambda}\left(\vartheta^{-} / 3\right)$ do not land at $b_{0}$. The usual techniques to prove this kind of result is based on a careful study of parabolic implosion (see for example the Orsay notes [DH1]). We will use a different approach based on Yoccoz inequality (see $[\mathrm{Hu}]$ or $[\mathrm{P}]$ ).

Let us first define $\mathcal{W}_{\vartheta}$ to be the connected component of $\mathcal{W}_{0} \backslash \overline{\mathcal{R}_{\lambda}\left(\vartheta^{-} / 3\right)} \cup$ $\overline{\mathcal{R}_{\lambda}\left(\vartheta^{+} / 3\right)}$ that contains the parameter rays $\mathcal{R}_{\lambda}(\theta)$, with $\left.\theta \in\right] \vartheta^{-} / 3, \vartheta^{+} / 3[$. We claim that the component $\mathcal{W}_{\vartheta}$ cannot intersect $M^{\prime}$. Indeed, proposition 10 shows that if $\mathcal{W}_{\vartheta}$ intersect $M^{\prime}$, there is a parameter $b^{\prime} \in \mathcal{W}_{\vartheta}$ such that $b^{\prime}$ is a tip of $M^{\prime}$ 
(tips of $M^{\prime}$ are dense in $\partial M^{\prime}$ ). But proposition 11 then shows that there are two parameter rays landing at $b^{\prime}$ whose angles are in $\Theta^{\prime}$. However, no angle between $\vartheta^{-}$and $\vartheta^{+}$can be written with only 1's and 2's.

Let us now assume that the parameter rays $\mathcal{R}_{\lambda}\left(\vartheta^{-} / 3\right)$ and $\mathcal{R}_{\lambda}\left(\vartheta^{+} / 3\right)$ land at $b_{0}$. Since $\mathcal{W}_{\vartheta} \cap M^{\prime}=\emptyset$, proposition 10 shows that for any $b \in M_{\lambda} \cap \mathcal{W}_{\vartheta}$, the fixed point $\alpha(b)$ is repelling and thus, has a rotation number. This rotation number is constant on any connected component $L$ of $M_{\lambda} \cap \mathcal{W}_{\vartheta}$. Besides, since $M_{\lambda}$ is connected, we necessarily have $b_{0} \in \bar{L}$. Since at $b_{0}$ the fixed point $\alpha(b)$ collapses with $\beta(b)$ and becomes a multiple fixed point, the multiplier at $\alpha(b)$ tends to 1 as $b$ tends to $b_{0}$, and the Yoccoz inequality shows that the rotation number of $\alpha(b)$ is $0 / 1$ for any $b \in L$. But in this case, for any $b \in L$ one of the two dynamical rays $R_{b}(0 / 1)$ or $R_{b}(1 / 2)$ has to land at $\alpha(b)$, which is impossible since they both land at $\beta(b) \neq \alpha(b)$. This gives the required contradiction.

Definition 17. For any dyadic angle $\vartheta$, we define the wake $\mathcal{W}_{\vartheta}$ to be the connected component of

$$
\mathbb{C} \backslash \overline{\mathcal{R}_{\lambda}\left(\vartheta^{-} / 3\right)} \cup \overline{\mathcal{R}_{\lambda}\left(\vartheta^{+} / 3\right)}
$$

that contains the parameter rays $\mathcal{R}_{\lambda}(\theta)$, with $\left.\theta \in\right] \vartheta^{-} / 3, \vartheta^{+} / 3[$.

Proposition 13. Given any dyadic angle $\vartheta=(2 p+1) / 2^{k}, k \geq 1,0<2 p+1<$ $2^{k}$, and any parameter $b \in \overline{\mathcal{W}_{\vartheta}}$, the dynamical rays $R_{b}\left(\vartheta^{-} / 3\right)$ and $R_{b}\left(\vartheta^{+} / 3\right)$ do not bifurcate and land at a common preimage of $\beta(b)$.

Proof. Let us assume that $b$ belongs to the parameter ray $\mathcal{R}_{\lambda}(\theta)$ and that the dynamical ray $R_{b}\left(\vartheta^{-} / 3\right)$ bifurcates. Then, note that the dynamical ray $R_{b}(\theta)$ bifurcates on $\omega_{2}^{\prime}(b)$. Hence, $R_{b}(3 \theta)$ contains the critical value $f_{b}\left(\omega_{2}(b)\right)$. Moreover, the dynamical ray $R_{b}\left(\vartheta^{-} / 3\right)$ bifurcates on a preimage of $\omega_{2}(b)$. Hence, there exists an integer $n \geq 0$ such that $f_{b}^{\circ n}\left(R_{b}\left(\vartheta^{-} / 3\right)\right)=R_{b}\left(3^{n-1} \vartheta^{-}\right)$bifurcates on $\omega_{2}(b)$. Since $R_{b}\left(\vartheta^{-} / 3\right) \subset U_{2}^{\prime}$, we necessarily have $n \geq 1$, and $R_{b}\left(3^{n} \vartheta^{-}\right)$contains the critical value $f_{b}\left(\omega_{2}(b)\right)$. This shows that the set of parameters $b \in \mathcal{W}_{0}$ where the dynamical ray $R_{b}\left(\vartheta^{-} / 3\right)$ bifurcates is precisely the union of parameter rays $\mathcal{R}_{\lambda}(\theta)$ where $\left.\theta \in\right] 1 / 6,2 / 3\left[\right.$ and $3 \theta=3^{n} \vartheta^{-} \bmod 1$ for some integer $n \geq 1$.

It is not difficult to check that for any $n \geq 1$, the angle $3^{n} \vartheta^{-}$mod 1 does not belong to the interval $\left[\vartheta^{-}, \vartheta^{+}\right]$. Besides, the parameter ray $\mathcal{R}_{\lambda}\left(3^{n} \vartheta^{-}\right)$lands at a tip of $M^{\prime}$ and this tip cannot be $b_{\vartheta}$ (see proposition 12). Hence, the set of parameter $b \in \mathcal{W}_{0}$ for which the dynamical ray $R_{b}\left(\vartheta^{-} / 3\right)$ do not bifurcate is a neighborhood of $\overline{\mathcal{W}_{\vartheta}}$. A similar argument shows that the set of parameter $b \in \mathcal{W}_{0}$ for which the dynamical ray $R_{b}\left(\vartheta^{+} / 3\right)$ do not bifurcate is a neighborhood of $\overline{\mathcal{W}_{\vartheta}}$. Since at $b_{\vartheta}$ the two dynamical rays $R_{b}\left(\vartheta^{-} / 3\right)$ and $R_{b}\left(\vartheta^{+} / 3\right)$ land at the common point $b_{\vartheta}$, we see that this property holds for any parameter $b$ in $\overline{\mathcal{W}_{\vartheta}}$. Finally, since $f_{b}^{\circ(k+1)}\left(R_{b}\left(\vartheta^{-} / 3\right)\right)=R_{b}(0 / 1)$ lands at $\beta(b)$, the landing point of the rays $R_{b}\left(\vartheta^{-} / 3\right)$ and $R_{b}\left(\vartheta^{+} / 3\right)$ is a preimage of $\beta(b)$.

Definition 18. Given any dyadic angle $\vartheta=(2 p+1) / 2^{k}, k \geq 1,0<2 p+1<2^{k}$, and any parameter $b \in \overline{\mathcal{W}_{\vartheta}}$, we define $W_{\vartheta}$ to be the connected component of

$$
\mathbb{C} \backslash\left(\overline{R_{b}\left(\vartheta^{-} / 3\right)} \cup \overline{R_{b}\left(\vartheta^{+} / 3\right)}\right)
$$

that contains the dynamical rays $\left.R_{b}(\theta), \theta \in\right] \vartheta^{-} / 3, \vartheta^{+} / 3[$. 
Proposition 14. Given any dyadic angle $\vartheta=(2 p+1) / 2^{k}, k \geq 1,0<2 p+1<$ $2^{k}$, and any parameter $b \in \mathcal{W}_{\vartheta}$, the co-critical point $\omega_{2}^{\prime}(b)$ belongs to the region $W_{\vartheta}(b)$ and the mapping $f_{b}^{\circ(k+1)}: W_{\vartheta}(b) \rightarrow V_{1}(b)$ is an isomorphism.

Proof. We have seen (proposition 13) that the boundary of the region $W_{\vartheta}(b)$ move holomorphically when $b$ moves in the wake $\mathcal{W}_{\vartheta}$. Furthermore, the co-critical point $\omega_{2}^{\prime}(b)$ cannot belong to this boundary since this would mean that $b$ is in the boundary of the wake $\mathcal{W}_{\vartheta}$. Hence, to see that for any to parameter $b \in \mathcal{W}_{\vartheta}$, the co-critical point $\omega_{2}^{\prime}(b)$ belongs to the region $W_{\vartheta}(b)$, it is enough to check it at one particular parameter $b \in \mathcal{W}_{\vartheta}$. This is clear as soon as $b$ is outside $M_{\lambda}$. Indeed, in this case $b$ belongs to a parameter ray $\mathcal{R}_{\lambda}(\theta)$ with $\left.\theta \in\right] \vartheta^{-} / 3, \vartheta^{+} / 3[$. Thus, $\omega_{2}^{\prime}(b)$ belongs to the dynamical ray $R_{b}(\theta) \subset W_{\vartheta}(b)$.

Since $f_{b}^{\circ(k+1)}: \mathbb{C} \rightarrow \mathbb{C}$ is a ramified covering, we know that for any connected component $W$ of $\left(f_{b}^{\circ(k+1)}\right)^{-1}\left(V_{1}(b)\right)$, the restriction $f_{b}^{\circ(k+1)}: W \rightarrow V_{1}(b)$ is also a ramified covering. Those components are the connected components of $\mathbb{C}$ minus the closure of the dynamical rays $R_{b}(\theta)$ where $3^{k+1} \theta \bmod 1$ is equal to 0 or $1 / 2$. It is not difficult to check that the region $W_{\vartheta}(b)$ contains no such ray. Thus, $f_{b}^{\circ(k+1)}: W_{\vartheta}(b) \rightarrow V_{1}(b)$ is a ramified covering. Since the boundary of $W_{\vartheta}(b)$ is mapped to the boundary of $V_{1}(b)$ with degree $1, f_{b}^{\circ(k+1)}: W_{\vartheta}(b) \rightarrow V_{1}(b)$ is an isomorphism.

\section{Copies of quadratic Julia sets in the parameter plane.}

In section 6 , we have defined the set

$$
X_{b}=\bigcup_{\theta \in \Theta} R_{b}(\theta)
$$

and we have proved that the mapping $h: \mathcal{W}_{0} \times X_{b_{1}} \rightarrow X_{b}$ defined by $h(b, z)=$ $\varphi_{b}^{-1} \circ \varphi_{b_{1}}(z)$ gives a holomorphic motion of the set $X_{b_{1}}$. In this section we fix once and for all a holomorphic motion $h: \mathcal{W}_{0} \times \mathbb{C} \rightarrow \mathbb{C}$ that coincide with the previous holomorphic motion on $\mathcal{W}_{0} \times X_{b_{1}}$. This can be done using Slodkowski's theorem (see [S1] or [D2]), because $\mathcal{W}_{0}$ is a simply connected Riemann surface.

We will also fix once and for all a dyadic angle $\vartheta=(2 p+1) / 2^{k}, k \geq 1$, $0<2 p+1<2^{k}$, and we will define $\vartheta^{-}, \vartheta^{+}, b_{\vartheta}, \mathcal{W}_{\vartheta}$ and $W_{\vartheta}(b)$ as in the previous section.

Definition 19. We define $\mathcal{X}_{\vartheta}$ to be the set of parameter rays

$$
\mathcal{X}_{\vartheta}=\bigcup_{\theta \in \Theta} \mathcal{R}_{\lambda}\left(\frac{\vartheta^{-}}{3}+\frac{\theta}{3^{k+1}}\right)
$$

Besides, we define $\mathcal{J}_{\vartheta}$ to be the set $\mathcal{J}_{\vartheta}=\overline{\mathcal{X}_{\vartheta}} \backslash \mathcal{X}_{\vartheta}$, where the closure is taken in $\mathbb{C}$. Finally, we define $\mathcal{K}_{\vartheta}$ to be the complement of the unbounded connected component of $\mathbb{C} \backslash \mathcal{J}_{\vartheta}$. 


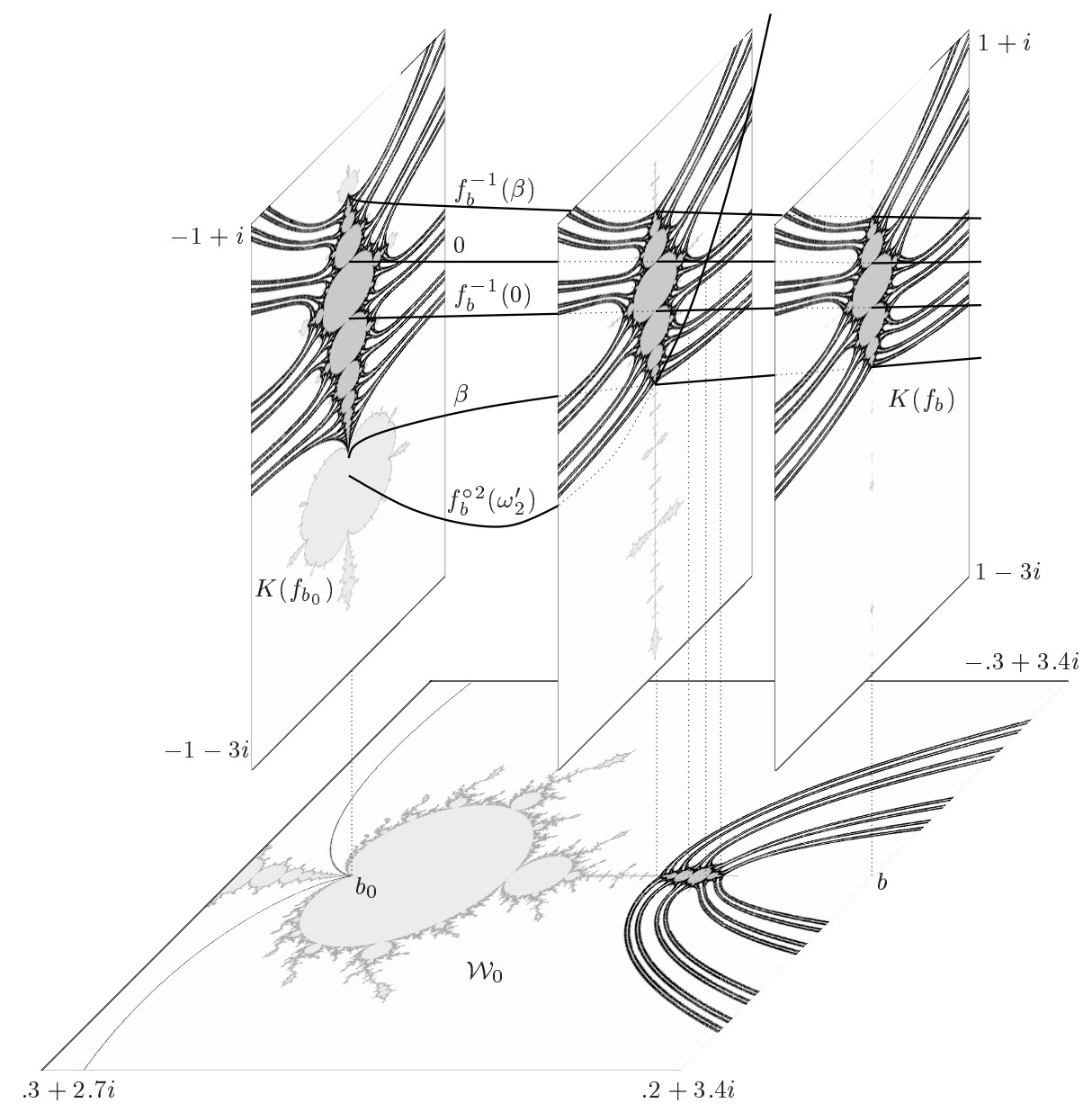

Fig. 13. The holomorphic motion for $\lambda=-1$.

Main Theorem. Let $\lambda \in S^{1}$ be a complex number of modulus 1 and $\vartheta \in \mathbb{R} / \mathbb{Z}$ be a dyadic angle. The set $\mathcal{K}_{\vartheta}$ is contained in $M_{\lambda} \cap \overline{\mathcal{W}_{\vartheta}}$, its boundary $\mathcal{J}_{\vartheta}$ is contained in the boundary of $M_{\lambda}$ and the parameter $b_{\vartheta}$ belongs to $\mathcal{J}_{\vartheta}$. Besides, there exist a quasi-conformal homeomorphism defined in a neighborhood of $\mathcal{K}_{\vartheta}$, sending $\mathcal{K}_{\vartheta}$ to $K\left(\lambda z+z^{2}\right)$.

Figure 13 suggests the main idea of the proof in the case $\lambda=-1, p=0$ and $k=1$, i.e., for $\vartheta=1 / 2$.

Proof. By definition of $\mathcal{X}_{\vartheta}$ and $\mathcal{J}_{\vartheta}$, we see that $\mathcal{X}_{\vartheta} \subset \overline{\mathcal{W}_{\vartheta}}$ and $\mathcal{J}_{\vartheta} \subset \partial M_{\lambda}$. Since $M_{\lambda}$ is full, we also have $\mathcal{K}_{\vartheta} \subset M_{\lambda}$. Finally, since $b_{\vartheta}$ is the landing point of the parameter ray $\mathcal{R}_{\lambda}\left(\vartheta^{-} / 3\right)$, we see that $b_{\vartheta} \in \mathcal{J}_{\vartheta}$. Hence, the only difficulty is proving that $\mathcal{K}_{\vartheta}$ is quasi-conformally homeomorphic to $K\left(\lambda z+z^{2}\right)$. 
Lemma 13. The mapping $H_{\vartheta}: \mathcal{W}_{0} \rightarrow \mathbb{C}$ defined by

$$
H_{\vartheta}(b)=h_{b}^{-1}\left[f_{b}^{\circ(k+1)}\left(\omega_{2}(b)\right)\right],
$$

is locally quasi-regular. Its restriction to the dyadic wake $\mathcal{W}_{\vartheta}$, is a homeomorphism which is locally quasi-conformal.

Proof. The argument we use is essentially due to Douady and Hubbard [DH2] (with some modifications). Let us first show that the restriction of $H_{\vartheta}$ to any open subset of $\mathcal{W}_{0}$ which is relatively compact in $\mathcal{W}_{0}$ is a quasi-regular mapping. It is enough to prove that there exists a $\kappa \in[0,1[$ such that the distributional derivatives of $H$ with respect to $b$ and $\bar{b}$ are locally in $L^{2}$ and satisfy

$$
\left\|\frac{\partial H_{\vartheta} / \partial \bar{b}}{\partial H_{\vartheta} / \partial \bar{b}}\right\|_{\infty} \leq \kappa<1
$$

Let us take the derivative with respect to $\bar{b}$ of the equation $h_{b} \circ H_{\vartheta}=f_{b}^{\circ(k+1)} \circ \omega_{2}$. Since $\partial h_{b} / \partial \bar{b}$ and $\partial\left(f_{b}^{\circ(k+1)} \circ \omega_{2}\right) / \partial \bar{b}$ identically vanish, we get

$$
\left.\left.\frac{\partial h_{b}}{\partial z}\right|_{H_{\vartheta}(b)} \frac{\partial H_{\vartheta}}{\partial \bar{b}}\right|_{b}+\left.\left.\frac{\partial h_{b}}{\partial \bar{z}}\right|_{H_{\vartheta}(b)} \frac{\partial \overline{H_{\vartheta}}}{\partial \bar{b}}\right|_{b}=0
$$

Thus,

$$
\left.\left.\left|\frac{\partial H_{\vartheta} / \partial \bar{b}}{\partial H_{\vartheta} / \partial b}\right|_{b}|=| \frac{\partial H_{\vartheta} / \partial \bar{b}}{\partial \overline{H_{\vartheta}} / \partial \bar{b}}\right|_{b}|=| \frac{\partial h_{b} / \partial \bar{z}}{\partial h_{b} / \partial z}\right|_{H_{\vartheta}(b)} \mid .
$$

The result follows by quasi-conformality of $h_{b}$.

Now, at every point $b \in \mathcal{W}_{0}$, the mapping $H_{\vartheta}$ has a local degree which is positive. To see that the restriction of $H_{\vartheta}$ to the wake $\mathcal{W}_{\vartheta}$ is proper, let us show that $H_{\vartheta}$ maps $\mathcal{W}_{\vartheta}$ (respectively $\partial \mathcal{W}_{\vartheta}$ ) to $V_{1}\left(b_{1}\right)$ (respectively $\partial V_{1}\left(b_{1}\right)$ ). Indeed, if $b \in \mathcal{W}_{\vartheta}$, then $\omega_{2}^{\prime}(b)$ belongs to the region $W_{\vartheta}(b)$ which is mapped isomorphically by $f_{b}^{\circ(k+1)}$ to $V_{1}(b)$ (see proposition 14 ). This shows that for any $b \in \mathcal{W}_{\vartheta}$,

$$
f^{\circ(k+1)}\left(\omega_{2}(b)\right)=f^{\circ(k+1)}\left(\omega_{2}^{\prime}(b)\right) \in V_{1}(b) .
$$

Moreover, by construction, for any $b \in \mathcal{W}_{0}$, we have $h_{b}\left(V_{1}\left(b_{1}\right)\right)=V_{1}(b)$. Since $h_{b}$ is a homeomorphism, we see that

$$
H_{\vartheta}(b)=h_{b}^{-1}\left[f_{b}^{\circ(k+1)}\left(\omega_{2}(b)\right)\right] \in h_{b}^{-1}\left(V_{1}(b)\right)=V_{1}\left(b_{1}\right) .
$$

Furthermore, the map $H_{\vartheta}$ is continuous in the whole wake $\mathcal{W}_{0}$ and in particular on the boundary of $\mathcal{W}_{\vartheta}$, i.e., on $\overline{\mathcal{R}_{\lambda}\left(\vartheta^{-}\right)} \cup \overline{\mathcal{R}_{\lambda}\left(\vartheta^{+}\right)}$. Since $h_{b}$ maps $R_{b_{1}}(0 / 1)$ (respectively $R_{b_{1}}(1 / 2)$ ) to $R_{b}(0 / 1)$ (respectively $R_{b}(1 / 2)$ ), we see that when $b \in \partial \mathcal{W}_{\vartheta}$, i.e., $\omega_{2}^{\prime}(b) \in \overline{R_{b}\left(\vartheta^{-}\right)} \cup \overline{R_{b}\left(\vartheta^{+}\right)}$, we have

$$
\begin{aligned}
H_{\vartheta}(b) & \in h_{b}^{-1}\left(f_{b}^{\circ 2}\left(\overline{R_{b}\left(\vartheta^{-}\right)} \cup \overline{R_{b}\left(\vartheta^{+}\right)}\right)\right)=h_{b}^{-1}\left(\overline{R_{b}(0 / 1)} \cup \overline{R_{b}(1 / 2)}\right) \\
& =\overline{R_{b_{1}}(0 / 1)} \cup \overline{R_{b_{1}}(1 / 2)}=\partial V_{1}\left(b_{1}\right) .
\end{aligned}
$$

Hence, the mapping $H_{\vartheta}: \mathcal{W}_{\vartheta} \rightarrow V_{1}\left(b_{1}\right)$ is a proper mapping. 
Let us now show that the topological degree of the restriction of $H_{\vartheta}$ to $\mathcal{W}_{\vartheta}$ is 1. Since $H_{\vartheta}$ is locally quasi-regular, the topological degree of $H_{\vartheta}$ at any point $b \in$ $\mathcal{W}_{\vartheta}$ is positive. Hence, it is enough to show that when $b$ turns once around $\mathcal{W}_{\vartheta}$, $H_{\vartheta}(b)$ turns once around $V_{1}\left(b_{1}\right)$. But this is straight forward since the point of potential $\eta$ on the parameter ray $\mathcal{R}_{\lambda}\left(\vartheta^{-}\right) / 3$ (respectively $\mathcal{R}_{\lambda}\left(\vartheta^{+}\right) / 3$ ) is mapped to the point of potential $3^{k+1} \eta$ on the dynamical ray $R_{b_{1}}(0 / 1)$ (respectively $\left.R_{b_{1}}(1 / 2)\right)$.

To conclude the proof of the main theorem, observe that $H_{\vartheta}\left(\mathcal{X}_{\vartheta}\right)=X_{b_{1}}$. Indeed, for any $\theta \in \Theta$,

$$
H_{\vartheta}\left(\mathcal{R}_{\lambda}\left(\frac{\vartheta^{-}}{3}+\frac{\theta}{3^{k+1}}\right)\right)=R_{b_{1}}\left(3^{k} \vartheta^{-}+\theta\right)=R_{b_{1}}(\theta) .
$$

Hence,

$$
H_{\vartheta}\left(\mathcal{J}_{\vartheta}\right)=H_{\vartheta}\left(\overline{\mathcal{X}_{\vartheta}} \backslash \mathcal{X}_{\vartheta}\right)=\overline{X_{b_{1}}} \backslash X_{b_{1}}=J_{b_{1}},
$$

and $H_{\vartheta}\left(\mathcal{K}_{\vartheta}\right)=K_{b_{1}}$. Since we know that $K_{b_{1}}$ is quasi-conformally homeomorphic to $K\left(\lambda z+z^{2}\right)$, the main theorem is proved.

We say that the family $f_{b}$ is stable at a parameter $b_{0}$ if and only if the Julia set $J\left(f_{b}\right)$ moves holomorphically in a neighborhood of $b_{0}$. The bifurcation locus of the family $f_{b}$ is defined to be the set of parameters where the family is not stable. Using the results obtained by Mañe, Sad and Sullivan in [MSS], one can prove that the bifurcation of the family $f_{b}, b \in \mathbb{C}$, is precisely the boundary of the connectedness locus $M_{\lambda}$. The following corollary is an immediate consequence of the previous theorem.

Corollary A. For each $\lambda=e^{2 i \pi \theta}$, the bifurcation locus of the one parameter family $f_{b}(z)=\lambda z+b z^{2}+z^{3}, b \in \mathbb{C}$, contains quasi-conformal copies of the quadratic Julia set $J\left(\lambda z+z^{2}\right)$.

\section{Non local connectivity in the parameter plane.}

We will now prove that when the Julia set $J\left(\lambda z+z^{2}\right)$ is not locally connected $M_{\lambda}$ is not locally connected.

Corollary B. If the Julia $J\left(\lambda z+z^{2}\right)$ is not locally connected, $M_{\lambda}$ is not locally connected.

Proof. The proof we will give here was explained to us by Lyubich and McMullen. Let us recall that if the continuous image of a locally connected compact set is Hausdorff, then it is locally connected. Thus, it is enough for our purposes to construct a continuous retraction from $M_{\lambda}$ to the set $\mathcal{K}_{\vartheta}$.

Let us first plough in the dynamical plane of $f_{b_{1}}$. Observe that the unbounded connected components of $\mathbb{C} \backslash \overline{X_{b_{1}}}$ are preimages of $V_{2}\left(b_{1}\right)$ by iterates of $f_{b_{1}}$. Since $V_{2}\left(b_{1}\right)$ is bounded by the dynamical rays $R_{b_{1}}(0 / 1)$ and $R_{b_{1}}(1 / 2)$ which both land at $\beta(b)$, we see that each unbounded connected component of $\mathbb{C} \backslash \overline{X_{b_{1}}}$ is bounded by two dynamical rays belonging to $X_{b_{1}}^{\prime}$ which land at a common preimage of $\beta\left(b_{1}\right)$. 
Harvesting in the parameter plane using $H_{\vartheta}$, we see that each unbounded connected components of $\mathbb{C} \backslash \overline{\mathcal{X}_{\vartheta}}$ is bounded by two parameter rays belonging to $\mathcal{X}_{\vartheta}$ which land at a common parameter which belong to $\mathcal{J}_{\vartheta}$. We can then define a retraction $\psi: \mathbb{C} \backslash \mathcal{X}_{\vartheta} \rightarrow \mathcal{K}_{\vartheta}$ which is the identity on $\mathcal{K}_{\vartheta}$ and sends every unbounded connected component $\mathcal{W}$ of $\mathbb{C} \backslash \overline{\mathcal{X}_{\vartheta}}$ to the landing point of the two parameter rays bounding $\mathcal{W}$.

This retraction is continuous. Indeed, every open set in $\mathcal{K}_{\vartheta}$ can be written $\mathcal{U} \cap \mathcal{K}_{\vartheta}$ with $\mathcal{U}$ open in $\mathbb{C} \backslash \mathcal{X}_{\vartheta}$. Then, the preimage of this open set is the union of $\mathcal{U}$ and the unbounded connected components of $\mathbb{C} \backslash \overline{\mathcal{X}_{\vartheta}}$ intersecting $\mathcal{U}$. This is clearly open.

The restriction of $\psi$ to $M_{\lambda} \subset \mathbb{C} \backslash \mathcal{X}_{\vartheta}$ gives the required retraction.

Let us finally prove that there exist values of $\lambda$ for which certain parameter rays have a non-trivial impression. In order to state our third corollary, we need to introduce some notations.

Definition 20. Given any complex number $\lambda$ of modulus 1 , we define $P_{\lambda}$ to be the quadratic polynomial $P_{\lambda}(z)=\lambda z+z^{2}$. We define $g_{P_{\lambda}}: \mathbb{C} \rightarrow[0,+\infty[$ to be its Green function and $\varphi_{P_{\lambda}}: \mathbb{C} \backslash K\left(P_{\lambda}\right) \rightarrow \mathbb{C} \backslash \overline{\mathbb{D}}$ to be its Böttcher coordinate. For any angle $\theta \in \mathbb{R} / \mathbb{Z}$, we define $R_{P_{\lambda}}(\theta)$ to be the dynamical ray of the polynomial $P_{\lambda}$ of angle $\theta$.

Definition 21. Let $\chi_{\Theta}: \mathbb{R} / \mathbb{Z} \rightarrow \mathbb{R} / \mathbb{Z}$ be the Cantor map (or devil staircase) which is constant on each connected component of $\mathbb{R} / \mathbb{Z} \backslash \Theta$ and is defined on $\Theta$ by:

$$
\chi_{\Theta}\left(\sum_{i \geq 1} \frac{\varepsilon_{i}}{3^{i}}\right)=\sum_{i \geq 1} \frac{\varepsilon_{i}}{2^{i}}, \quad \text { where } \quad \varepsilon_{i} \in\{0,1\} .
$$

Corollary C. Given any complex number $\lambda$ of modulus 1 and dyadic angle $\vartheta=(2 p+1) / 2^{k}$ and any angle $\theta \in \Theta$, the accumulation set of the parameter ray $\mathcal{R}_{\lambda}\left(\vartheta^{-} / 3+\theta / 3^{k+1}\right)$ is reduced to a point if and only if the accumulation set of the quadratic ray $R_{P_{\lambda}}\left(\chi_{\Theta}(\theta)\right)$ is reduced to a point.

The following proof was explained to us by Douady.

Proof. The proof of the main theorem provides a homeomorphism

$$
H_{\vartheta}: \mathcal{W}_{\vartheta} \rightarrow V_{1}\left(b_{1}\right)
$$

which maps each parameter ray $\mathcal{R}_{\lambda}\left(\vartheta^{-} / 3+\theta / 3^{k+1}\right), \theta \in \Theta$, to the dynamical ray $R_{b_{1}}(\theta)$. Hence, it is enough to prove that for any $\theta \in \Theta$, the accumulation set of the dynamical ray $R_{b_{1}}(\theta)$ is reduced to a point if and only if the accumulation set of the quadratic ray $R_{P_{\lambda}}\left(\chi_{\Theta}(\theta)\right)$ is reduced to a point.

Let us recall that the mapping $f_{b_{1}}: U_{b_{1}}^{\prime} \rightarrow U_{b_{1}}$ is a quadratic-like mapping hybrid conjugate to the quadratic polynomial $P_{\lambda}$ (see proposition 5 and figure 3 ). To fix the ideas, we choose a potential $\eta_{0}>0$, we set $U_{P_{\lambda}}=\left\{z \in \mathbb{C} \mid g_{P_{\lambda}}(z)<\right.$ $\left.2 \eta_{0}\right\}$ and $U_{P_{\lambda}}^{\prime}=\left\{z \in \mathbb{C} \mid g_{P_{\lambda}}(z)<\eta_{0}\right\}$. Then, we choose a quasi-conformal homeomorphism $\psi: U_{b_{1}} \rightarrow U_{P_{\lambda}}$ that conjugates $f_{b_{1}}: U_{b_{1}}^{\prime} \rightarrow U_{b_{1}}$ to $P_{\lambda}: U_{P_{\lambda}}^{\prime} \rightarrow$ $U_{P_{\lambda}}$ and that sends the segment of dynamical ray $U_{b_{1}} \cap R_{b_{1}}(0 / 1)$ onto $U_{P_{\lambda}} \cap$ 
$R_{P_{\lambda}}(0 / 1)$. We will construct a continuous mapping $\psi_{\Theta}: U_{b_{1}} \backslash K_{b_{1}} \rightarrow U_{P_{\lambda}} \backslash K\left(P_{\lambda}\right)$ which semi-conjugates $f_{b_{1}}: U_{b_{1}}^{\prime} \rightarrow U_{b_{1}}$ to $P_{\lambda}: U_{P_{\lambda}}^{\prime} \rightarrow U_{P_{\lambda}}$ and which maps $R_{b_{1}}(\theta) \cap U_{b_{1}}, \theta \in \Theta$, to $R_{P_{\lambda}}\left(\chi_{\Theta}(\theta)\right)$. We will then prove that the distance, for the hyperbolic metric on $\mathbb{C} \backslash K\left(P_{\lambda}\right)$, between $\psi(z)$ and $\psi_{\Theta}(z)$, is uniformly bounded independently on $z \in U_{P_{\lambda}} \backslash K\left(P_{\lambda}\right)$. It easily follows that the accumulation sets of $\psi\left(R_{b_{1}}(\theta)\right)$ and $\psi_{\Theta}\left(R_{b_{1}}(\theta)\right)=R_{P_{\lambda}}\left(\chi_{\Theta}(\theta)\right)$ are equal. Since $\psi: U_{b_{1}} \rightarrow U_{P_{\lambda}}$ is a homeomorphism, this will complete the proof of corollary C.

Let us now fill in the details. We will need to work with the universal coverings of $V_{b_{1}}=\mathbb{C} \backslash K_{b_{1}}$ and $V_{P_{\lambda}}=\mathbb{C} \backslash K\left(P_{\lambda}\right)$. To write things correctly and to avoid nasty traps, we need to choose basepoints. We choose $z_{0}$ (respectively $z_{1}$ ) to be the point of potential $G\left(b_{1}\right)$ (respectively $G\left(b_{1}\right) / 3$ ) on the dynamical ray $R_{b_{1}}(0 / 1)$. We then define $\pi_{b_{1}}: \widetilde{V}_{b_{1}} \rightarrow V_{b_{1}}$ to be the universal covering of $V_{b_{1}}$ with basepoint at $z_{0}$. We choose $\widetilde{R}$ to be a lift of $R_{b_{1}}(0 / 1)$ and we define $\widetilde{z}_{0}$ (respectively $\widetilde{z}_{1}$ ) to be the point of $\widetilde{R}$ which is in the fiber of $z_{0}$ (respectively $z_{1}$ ). Next, we define $\widetilde{U}_{b_{1}}=\pi_{b_{1}}^{-1}\left(U_{b_{1}} \backslash K_{b_{1}}\right)$ and $\widetilde{U}_{b_{1}}^{\prime}=\pi_{b_{1}}^{-1}\left(U_{b_{1}}^{\prime} \backslash K_{b_{1}}\right)$. Then, we call $\widetilde{f}_{b_{1}}: \widetilde{U}_{b_{1}}^{\prime} \rightarrow \widetilde{U}_{b_{1}}$ the lift of $f_{b_{1}}: U_{b_{1}}^{\prime} \rightarrow U_{b_{1}}$ that sends $\widetilde{z}_{1}$ to $\widetilde{z}_{0}$ :

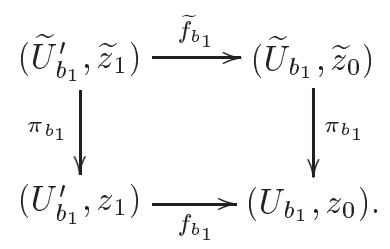

Finally, observe that the fundamental group of $V_{b_{1}}$ is a cyclic group that acts on $\widetilde{V}_{b_{1}}$. We call $\gamma_{b_{1}}: \widetilde{V}_{b_{1}} \rightarrow \widetilde{V}_{b_{1}}$ the automorphism of $\widetilde{V}_{b_{1}}$ that corresponds to turning once around $K_{b_{1}}$ counter-clockwise. Since $f_{b_{1}}: U_{b_{1}}^{\prime} \rightarrow U_{b_{1}}$ maps a loop that turns once around $K_{b_{1}}$ counter-clockwise to a loop that turns twice around $K_{b_{1}}$ counter-clockwise, we see that $\tilde{f}_{b_{1}} \circ \gamma_{b_{1}}=\gamma_{b_{1}}^{\circ 2} \circ \widetilde{f}_{b_{1}}$.

Similarly, we define $w_{0}$ (respectively $w_{1}$ ) to be the point of potential $\eta_{0}$ (respectively $\left.\eta_{0} / 2\right)$ on the quadratic ray $R_{P_{\lambda}}(0 / 1)$. We define $\pi_{P_{\lambda}}: \widetilde{V}_{P_{\lambda}} \rightarrow V_{P_{\lambda}}$ to be the universal covering with basepoint at $w_{0}$. In this case, we can give an explicit formula. We identify $\widetilde{V}_{P_{\lambda}}$ with the right half-plane $\mathbb{H}=\{z \in \mathbb{C} \mid \operatorname{Re}(z)>0\}$ and we set $\pi_{P_{\lambda}}=\varphi_{P_{\lambda}}^{-1} \circ$ exp. The real axis projects to the quadratic ray $R_{P_{\lambda}}(0 / 1)$. Thus, we define $\widetilde{w}_{0}=\eta_{0}$ and $\widetilde{w}_{1}=\eta_{0} / 2$, so that $\pi_{P_{\lambda}}\left(\widetilde{w}_{0}\right)=w_{0}$ and $\pi_{P_{\lambda}}\left(\widetilde{w}_{1}\right)=$ $w_{1}$. We define

$$
\widetilde{U}_{P_{\lambda}}=\pi_{P_{\lambda}}^{-1}\left(U_{P_{\lambda}} \backslash K\left(P_{\lambda}\right)\right)=\left\{z \in \mathbb{H} \mid \operatorname{Re}(z)<2 \eta_{0}\right\}
$$

and

$$
\widetilde{U}_{P_{\lambda}}^{\prime}=\pi_{P_{\lambda}}^{-1}\left(U_{P_{\lambda}}^{\prime} \backslash K\left(P_{\lambda}\right)\right)=\left\{z \in \mathbb{H} \mid \operatorname{Re}(z)<\eta_{0}\right\} .
$$

The lift of $P_{\lambda}: V_{P_{\lambda}} \rightarrow V_{P_{\lambda}}$ that sends $\widetilde{w}_{1}$ to $\widetilde{w}_{0}$ is the map $\widetilde{w} \mapsto 2 \widetilde{w}$. Finally, the automorphism of $\mathbb{H}$ that corresponds to turning once around $K\left(P_{\lambda}\right)$ counterclockwise is the translation $z \mapsto z+2 i \pi$.

Next, a quasi-conformal homeomorphism $\psi: U_{b_{1}} \rightarrow U_{P_{\lambda}}$, that conjugates $f_{b_{1}}: U_{b_{1}}^{\prime} \rightarrow U_{b_{1}}$ to $P_{\lambda}: U_{P_{\lambda}}^{\prime} \rightarrow U_{P_{\lambda}}$ and that sends the segment of dynamical ray $U_{b_{1}} \cap R_{b_{1}}(0 / 1)$ onto $U_{P_{\lambda}} \cap R_{P_{\lambda}}(0 / 1)$, can be lifted to a quasi-conformal 
homeomorphism $\widetilde{\psi}: \widetilde{U}_{b_{1}} \rightarrow \widetilde{U}_{P_{\lambda}}$ that sends $\widetilde{z}_{0}$ to $\widetilde{w}_{0}$. Then $\widetilde{\psi}$ sends $\widetilde{R} \cap \widetilde{U}_{b_{1}}$ to $\mathbb{R} \cap \widetilde{U}_{P_{\lambda}}$. Hence, it also sends $\widetilde{z}_{1}$ to $\widetilde{w}_{1}$, and it is not difficult to see that it conjugates $\widetilde{f}_{b_{1}}$ to multiplication by $2: \widetilde{\psi} \circ \widetilde{f}_{b_{1}}=2 \widetilde{\psi}$.

We now come to the construction of the semi-conjugacy $\psi_{\Theta}$. First, consider the increasing homeomorphism $h:\left[G\left(b_{1}\right), 3 G\left(b_{1}\right)\right] \rightarrow\left[\eta_{0}, 2 \eta_{0}\right]$ defined by

$$
h=g_{P_{\lambda}} \circ \psi \circ \varphi_{b_{1}}^{-1} \circ \exp
$$

$\left(h(\eta)\right.$ is the potential in $\mathbb{C} \backslash K\left(P_{\lambda}\right)$ of the image by $\psi$ of the point of potential $\eta$ on the dynamical ray $\left.R_{b_{1}}(0 / 1)\right)$. Then, define the continuous mapping $\psi_{\Theta}$ : $\overline{U_{b_{1}}} \backslash U_{b_{1}}^{\prime} \rightarrow \overline{U_{P_{\lambda}}} \backslash U_{P_{\lambda}}^{\prime}$ in the following way:

- on $U_{b_{1}}^{\prime \prime}$, the map $\psi_{\Theta}$ is constantly equal to $\varphi_{P_{\lambda}}^{-1}\left(e^{\eta_{0}}\right)$, i.e., the point of potential $\eta_{0}$ on the dynamical ray $R_{P_{\lambda}}(0 / 1)$

- on $\overline{U_{b_{1}}} \backslash\left(U_{b_{1}}^{\prime} \cup U_{b_{1}}^{\prime \prime}\right)$ the map $\psi_{\Theta}$ sends the point $\varphi_{b_{1}}^{-1}\left(e^{\eta+2 i \pi \theta}\right)$ to the point $\varphi_{P_{\lambda}}^{-1}\left(e^{h(\eta)+2 i \pi \chi_{\Theta}(\theta)}\right)$.

Observe that on the boundary of $U_{b_{1}}^{\prime}$, we have $\psi_{\Theta} \circ f_{b_{1}}=P_{\lambda} \circ \psi_{\Theta}$. Now, consider

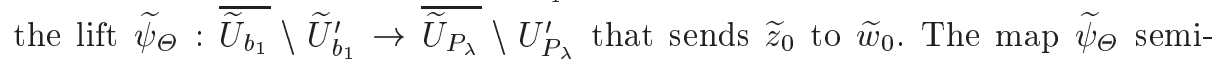
conjugates $\tilde{f}_{b_{1}}$ to multiplication by 2 on the boundary of $\widetilde{U}_{b_{1}}^{\prime}$. Thus, we can extend it continuously to $\widetilde{U}_{b_{1}}$ using the formula:

$$
\widetilde{\psi}_{\Theta}(\widetilde{z})=\frac{1}{2^{n}} \widetilde{\psi}_{\Theta}\left(\widetilde{f}_{b_{1}}^{\circ n}(\widetilde{z})\right)
$$

where $n$ is chosen so that $\tilde{f}_{b_{1}}^{\circ n}(\widetilde{z})$ belongs to $\overline{\widetilde{U}_{b_{1}}} \backslash \widetilde{U}_{b_{1}}^{\prime}$. An easy induction shows that $\widetilde{\psi}_{\Theta} \circ \gamma_{1}=\widetilde{\psi}_{\Theta}+2 i \pi$. Hence, $\widetilde{\psi}_{\Theta}$ projects to a continuous map $\psi_{\Theta}: U_{b_{1}} \backslash K_{b_{1}} \rightarrow$ $U_{P_{\lambda}} \backslash K\left(P_{\lambda}\right)$ that semi-conjugates $f_{b_{1}}: U_{b_{1}}^{\prime} \rightarrow U_{b_{1}}$ to $P_{\lambda}: U_{P_{\lambda}}^{\prime} \rightarrow U_{P_{\lambda}}$.

We claim that for any $\theta \in \Theta, \psi_{\Theta}$ maps $R_{b_{1}}(\theta) \cap U_{b_{1}}$ homeomorphically onto $R_{P_{\lambda}}\left(\chi_{\Theta}(\theta) \cap U_{P_{\lambda}}\right)$. Indeed, set $A_{0}=\overline{U_{b_{1}}} \backslash U_{b_{1}}^{\prime}$ and for $n \geq 0$ define recursively $A_{n+1}=f_{b_{1}}^{-1}\left(A_{n}\right)$. Similarly, define $B_{n}$ to be the annulus

$$
B_{n}=\left\{z \in \mathbb{C} \backslash K\left(P_{\lambda}\right) \mid \eta_{0} / 2^{n} \leq g_{P_{\lambda}}(z) \leq \eta_{0} / 2^{n-1}\right\}
$$

By construction, for every $\theta \in \Theta$, we have $\psi_{\Theta}\left(R_{b_{1}}(\theta) \cap A_{0}\right)=R_{P_{\lambda}}\left(\chi_{\Theta}(\theta)\right) \cap B_{0}$. Besides, since $\psi_{\Theta}$ semi-conjugates $f_{b_{1}}$ and $P_{\lambda}$, we see that for every $n \geq 0$ and every $\theta \in \Theta, \psi_{\Theta}\left(R_{b_{1}}(\theta) \cap A_{n}\right)$ is contained in the intersection of the annulus $B_{n}$ with a ray of $P_{\lambda}$. Since $\psi_{\Theta}$ is continuous, the whole set $\psi_{\Theta}\left(R_{b_{1}}(\theta) \cap U_{b_{1}}\right)$ is contained in a single ray of $P_{\lambda}$, i.e., the ray $R_{P_{\lambda}}\left(\chi_{\Theta}(\theta)\right)$. The point of potential $\eta$ is mapped to the point of potential $h\left(3^{n} \eta\right) / 2^{n}$, where $n$ is chosen so that $G\left(b_{1}\right) \leq 3^{n} \eta \leq 3 G\left(b_{1}\right)$. This shows that $\psi_{\Theta}: R_{b_{1}}(\theta) \cap U_{b_{1}} \rightarrow R_{P_{\lambda}}\left(\chi_{\Theta}(\theta) \cap U_{P_{\lambda}}\right)$ is a homeomorphism.

Let us finally show that the distance, for the hyperbolic metric on $\mathbb{C} \backslash K\left(P_{\lambda}\right)$, between $\psi(z)$ and $\psi_{\Theta}(z)$, is uniformly bounded independently on $z \in U_{b_{1}} \backslash K_{b_{1}}$. It is enough to prove that for any $\widetilde{z} \in \widetilde{U}_{b_{1}}$, the hyperbolic distance in $\mathbb{H}$ between $\widetilde{\psi}(\widetilde{z})$ and $\widetilde{\psi}_{\Theta}(\widetilde{z})$ is uniformly bounded. Since $\widetilde{\psi} \circ \widetilde{f}_{b_{1}}=2 \widetilde{\psi}$ and $\widetilde{\psi}_{\Theta} \circ \widetilde{f}_{b_{1}}=2 \widetilde{\psi}_{\Theta}$, and since multiplication by 2 is an isometry for the hyperbolic metric on $\mathbb{H}$, it is enough to prove the statement on the intersection of $\widetilde{U}_{b_{1}} \backslash \widetilde{U}_{b_{1}}^{\prime}$ with a fundamental 
domain for $\gamma_{1}$. This is immediate since the closure of such a set is compact in $\widetilde{V}_{b_{1}}$ and the mappings $\widetilde{\psi}$ and $\widetilde{\psi}_{\Theta}$ are continuous on $\widetilde{V}_{b_{1}}$.

It now follows that we can extend $\psi_{\Theta}$ continuously to $K_{b_{1}}$ by setting $\left.\psi_{\Theta}\right|_{K_{b_{1}}}=$ $\left.\psi\right|_{K_{b_{1}}}$. Given $\theta \in \Theta$ consider the restriction of $\psi_{\Theta}$ to $\left(R_{b_{1}}(\theta) \cap \overline{U_{b_{1}}}\right) \cup K_{b_{1}}$. Since this map is injective continuous and the domain is compact, it is necessarily a homeomorphism. Notice that the closure of $R_{b_{1}}(\theta) \cap \overline{U_{b_{1}}}$ in $\mathbb{C}$ equals the closure taken in $\left(R_{b_{1}}(\theta) \cap \overline{U_{b_{1}}}\right) \cup K_{b_{1}}$. Similarly the closure of $R_{P_{\lambda}}\left(\chi_{\Theta}(\theta)\right) \cap \overline{U_{P_{\lambda}}}$ in $\mathbb{C}$ equals the closure taken in $\left(R_{P_{\lambda}}\left(\chi_{\Theta}(\theta)\right) \cap \overline{U_{P_{\lambda}}}\right) \cup K\left(P_{\lambda}\right)$. In particular $\left.\psi_{\Theta}\right|_{K_{b_{1}}}=$ $\left.\psi\right|_{K_{b_{1}}}$ provides a homeomorphism, mapping the impression of $R_{b_{1}}(\theta)$ onto the impression of $R_{P_{\lambda}}\left(\chi_{\Theta}(\theta)\right)$.

Let us now consider the function $\theta_{2}:(\mathbb{R} \backslash \mathbb{Q}) / \mathbb{Z} \rightarrow(\mathbb{R} \backslash \mathbb{Q}) / \mathbb{Z}$ defined in the following way: for any irrational angle, first choose the representative $t \in] 0,1[$, then define

$$
\theta_{2}(t)=\sum_{0<p / q<t} \frac{1}{2^{q+1}}
$$

The sum is taken over all pairs $(p, q)$ such that $0<p / q<t$, whether $p$ and $q$ are relatively prime or not. Douady proved that the set of complex numbers $\lambda=e^{2 i \pi t}, t \in(\mathbb{R} \backslash \mathbb{Q}) / \mathbb{Z}$, for which the accumulation set of the quadratic ray $R_{P_{\lambda}}\left(\theta_{2}(t)\right)$ is not reduced to a point, is a dense $G_{\delta}$ subset of $S^{1}$. The proof can be found in $[\mathrm{S} \varnothing]$.

Next, observe that for each $t \in \mathbb{R} \backslash \mathbb{Q}$, there is exactly one angle $\theta_{3}(t) \in \Theta$ which is mapped to $\theta_{2}(t)$ by $\chi_{\Theta}$ :

$$
\theta_{3}(t)=\sum_{0<p / q<t} \frac{1}{3^{q+1}}
$$

The previous corollary shows that when the accumulation set of the quadratic ray $R_{P_{\lambda}}\left(\theta_{2}(t)\right)$ is not reduced to a point, then the accumulation set of the parameter ray $\mathcal{R}_{\lambda}\left(2 / 9+\theta_{3}(t) / 9\right)$ is also not reduced to a point. This shows that the set of complex numbers $\lambda$ of modulus 1 for which at least one of the parameter rays $\mathcal{R}_{\lambda}(\theta) \subset \mathbb{C} \backslash M_{\lambda}$ has an accumulation set not reduced to a point, contains a dense $G_{\delta}$ subset of $S^{1}$.

Acknowledgements. We are very grateful to Bodil Branner, Adrien Douady, John Hubbard and Carsten Petersen for encouraging us. We would like to thank the department of mathematics of the University at Cornell for its hospitality during the academic year 1997-1998. There, we drew the first pictures showing evidence of the existence of Julia sets in parameter spaces. We would also like to thank the departments of mathematics of Technical University of Denmark and of Université Paul Sabatier. This research was supported by the french embassy in Denmark: it made possible the exchanges that took place at the time this article was written.

\section{References}

[Br] Branner, B.: Puzzles and Parapuzzles of quadratic and Cubic Polynomials. Proceedings of Symposia in Applied Math. 49, 31-69 (1994)

[BH1] Branner, B. and Hubbard, J.: The iteration of cubic polynomials, Part I: The global topology of parameter space. Acta Math. 160, 143-206 (1988)

[BH2] Branner, B. and Hubbard, J.: The iteration of cubic polynomials, Part II: Patterns and parapatterns. Acta Math. 169, 229-325 (1992) 
[D1] Douady, A.: Does a Julia set depend continuously on the Polynomial ? Proceedings of Symposia in Applied Math. 49, 91-139 (1994)

[D2] Douady, A.: Prolongement de mouvements holomorphes [d'après Slodkowski et autres]. Séminaire Bourbaki, Astérisque 227, 7-20 (1993)

[DH1] Douady, A. and Hubbard, J.: Étude dynamique des polynômes complexes I \& II. Publ. Math. d'Orsay, (1984-85)

[DH2] Douady, A. and Hubbard, J.: On the dynamics of polynomial-like mappings. Ann. Sci. Éc. Norm. Sup. 18, 287-343 (1985)

[EY] Epstein, A. and Yampolsky, M.: Geography of the Cubic Connectedness Locus: Intertwining Surgery. Ann. Sci. Éc. Norm. Sup. 32, 151-185 (1999)

[Ha] Haïssinsky, P.: Chirurgie parabolique. C. R. Acad. Sc. Paris 327, 195-198 (1998)

[Hu] Hubbard, J.: Local connectivity of Julia sets and bifurcation loci: three theorems of J.C. Yoccoz. Topological methods in modern mathematics (Stony Brook, NY, 1991), Publish or Perish, Houston, TX, 467-511 (1993)

[K] Kiwi, J.: Rational Rays and Critical Portraits of Complex Polynomials. SUNY at Stony Brook IMS preprint, 1997/15

[La] Lavaurs, P.: Systèmes dynamiques holomorphes, Explosion de points périodiques paraboliques. Thèse de doctorat, Université Paris-Sud in Orsay, (1989)

[MSS] Mañe, R., Sad, P. and Sullivan, D.: On the dynamics of rational maps. Ann. Sci. Éc. Norm. Sup. 16, 193-217 (1983)

[M] Milnor, J.: Local connectivity of Julia sets: Expository Lectures, In: The Mandelbrot Set, Theme and Variations, ed. Tan Lei, London Math. Soc. Lect. Note 274: Cambridge Univ. Press, 67-116 (2000).

[Nai] Naishul, V.I.: Topological Invariants of analytic and area preserving mappings and their applications to analytic differential equations in $\mathbb{C}^{2}$ and $\mathbb{C P}^{2}$. Trans. Moscow Math. Soc. 42, 239-250 (1983).

[NK] Nakane, S. and Komori, Y.: Non-landing of Stretching Rays for the Family of Real Cubic Polynomials. Manuscript.

[PM] Pérez-Marco, R.: Fixed points and circle maps; Acta Math., 179, 243-294 (1997)

[P] Petersen,C.: On the Pommerenke-Levin-Yoccoz inequality, Ergod. Th. Dynam. Sys. 13, 785-806 (1993)

[R] Roesch, P.: Topologie locale des méthodes de Newton cubiques: plan dynamique., C. R. Acad. Sc. 326, 1221-1226 (1998)

[Sl] Slodkowski, Z.: Extensions of holomorphic motions, Ann. Scuola Norm. Sup. Pisa Cl. Sci. 22, 185-210 (1995)

[Sø] Sørensen, D.: Infinitely renormalizable quadratic polynomials, with non-locally connected Julia set. J. Geom. Anal. 10, 169-206 (2000)

[TL] Tan Lei.: Similarity between the Mandelbrot set and Julia sets. Commun. Math. Phys. 134, 587-617 (1990)

[Y] Yoccoz, J.C.: Petits diviseurs en dimension 1. Astérisque 231, (1995)

[Z1] Zakeri, S.: On Dynamics of Cubic Siegel Polynomials. SUNY at Stony Brook IMS preprint, 1998/4

[Z2] Zakeri, S.: Dynamics of Cubic Siegel Polynomials. Commun. Math. Phys. 206, 185233 (1999).

Communicated by Ya. G. Sinai 